\title{
A Convolutional Neural Network-based approach for the Rapid Characterization of Molecularly Diverse Natural Products
}

Raphael Reher ${ }^{+\dagger}$, Hyun Woo Kim ${ }^{+, \dagger}$, Chen Zhang ${ }^{+,+\S}$, Huanru Henry Mao ${ }^{\S}$, Mingxun Wang ${ }^{\ddagger}$, Louis-Félix Nothias ${ }^{\ddagger}$, Andres Mauricio Caraballo-Rodriguez ${ }^{\ddagger}$ Evgenia Glukhov ${ }^{\dagger}$, Bahar Teke ${ }^{\dagger}$, Tiago Leao ${ }^{\dagger}$, Kelsey L. Alexander ${ }^{\dagger, \perp}$ Brendan M. Duggan ${ }^{\ddagger}$, Ezra L. Van Everbroeck", Pieter C. Dorrestein $^{\ddagger}$, Garrison W. Cottrell ${ }^{\star}$, William H. Gerwick* ${ }^{\star \dagger, \ddagger}$

†Center for Marine Biotechnology and Biomedicine, Scripps Institution of Oceanography, University of California, San Diego, 9500 Gilman Drive, La Jolla, California 92093, United States

§Department of Computer Science and Engineering, UC San Diego, 9500 Gilman Drive, La Jolla, California 92093, United States

‡Skaggs School of Pharmacy and Pharmaceutical Sciences, UC San Diego, 950o Gilman Drive, La Jolla, California 92093, United States

${ }^{\perp}$ Department of Chemistry and Biochemistry, UC San Diego, 9500 Gilman Drive, La Jolla, California 92093, United States

"Director's Office, Scripps Institution of Oceanography, University of California, San Diego, 9500 Gilman Drive, La Jolla, California 92093, United States 


\section{Table of Contents}

Content

Page

Experimental procedures

S2

Structure elucidation of symplocolide A (1) $\quad$ S17

Figure $\mathrm{S}_{5}:{ }^{1} \mathrm{H}$ NMR spectrum $\left(500 \mathrm{MHz} \mathrm{CDCl}_{3}\right)$ of $\mathbf{1} \quad S 17$

Figure S6: ${ }^{13} \mathrm{C}$ NMR spectrum (125 $\left.\mathrm{MHz} \mathrm{CDCl}_{3}\right)$ of 1

Figure $\mathrm{S}_{7}:{ }^{1} \mathrm{H}-{ }^{1} \mathrm{H}$ COSY NMR spectrum (500 $\mathrm{MHz} \mathrm{CDCl}_{3}$ ) of $\mathbf{1} \quad S 18$

Figure S8: ${ }^{1 \mathrm{H}-13 \mathrm{C} \text {-edited HSQC NMR spectrum (500 } \mathrm{MHz} \mathrm{CDCl}_{3} \text { ) of } 1} \quad \mathrm{~S} 18$

Figure S9: ${ }^{1} \mathrm{H}-13 \mathrm{C}$ HMBC NMR spectrum (500 $\mathrm{MHz} \mathrm{CDCl}_{3}$ ) of 1 Si9

Figure S10: ${ }^{\mathrm{H}} \mathrm{-}-\mathrm{H}$ ROESY NMR spectrum (500 $\mathrm{MHz} \mathrm{CDCl}_{3}$ ) of 1

Table S1: ${ }^{1} \mathrm{H}$ and ${ }^{13} \mathrm{C}$ NMR spectroscopic data of 1 in $\mathrm{CDCl}_{3} \quad$ S2O

Figure S11: High-Resolution LCMS ESI-ToF data for $1 \quad$ S21

Table S2: ${ }^{13}$ C NMR data comparing swinholide A (4), 1, and luminaolide B (3) $\quad$ S21

Table S3: ${ }^{1} \mathrm{H}$ NMR data comparing 4, 1, and $3 \quad$ S23

\section{Cytotoxicity assays}

Figure S3: Cytotoxicity assay of crude extract, and various purification stages of the most active fractions H + I from Symploca sp. $\quad \mathrm{S}_{15}$

$\begin{array}{ll}\text { Figure S31: Cytotoxicity assay of } 1 & \text { S41 }\end{array}$

Structure elucidation of known and new swinholides, samholides, and symplocolides

Scheme S1: Chemical classification scheme of swinholide-related macrolides $\quad$ S14

Figure S12: MS²-based molecular networking of the cytotoxic fractions H + I $\quad$ S24

Figure $\mathrm{S}_{4}$ : MS $\mathrm{MS}^{2}$ Fragmentation spectra of $4 \quad \mathrm{~S} 6$

Figure S13: MS² Fragmentation spectra of samholide A/B (5/6) $\quad$ S25

Figure S14-S16: MS² Fragmentation spectra of samholide C/D (7/8) S26

Figure S17-20: MS ${ }^{2}$ Fragmentation spectra of samholide E/F/G (9/10/11) S29

Figure S21: MS² Fragmentation spectra of samholide H (12) $\quad$ S33

Figure S22: MS ${ }^{2}$ Fragmentation spectra of samholide I (13) S34

Figure S23-25: MS ${ }^{2}$ Fragmentation spectra of putatively new samholide analogs $\quad$ S35

Figure S26-28: MS² Fragmentation spectra of putatively new swinholide analogs $\quad \mathrm{S}_{3} 8$

Figure S29-30: MS² Fragmentation spectra of putatively new symplocolide analogs $\quad$ S40

\section{SMART 2.0 robustness tests and mixture analysis}

Figure S1: Effect of NMR solvents on SMART results $\quad$ S12

Figure S2: Effect of calculated (predicted in different solvents) spectra on SMART results $\mathrm{S}_{13}$

Figure S32: SMART 2.0 results on the new gracilioether $L \quad S 41$

Figure S33: Mixture analysis of cyclomarin A-containing crude extract and

comparison of cyclomarin A isolated by Duggan et al. and Renner et al. by SMART 2.0 S42

$\begin{array}{ll}\text { Supplementary references } & \text { S43 }\end{array}$ 


\section{General Experimental Procedures}

Chemical reagents were purchased from Acros, Fluka, Sigma-Aldrich, or TCI. Deuterated NMR solvents were purchased from Cambridge Isotope Laboratories.

${ }^{1} \mathrm{H}$ NMR and 2D NMR spectra were collected on a Bruker Avance III DRX-6oo NMR with a 1.7 mm dual tune TCI cyroprobe (6oo and $150 \mathrm{MHz}$ for ${ }^{1} \mathrm{H}$ and ${ }^{13} \mathrm{C}$ NMR, respectively) and a JEOL ECZ 500 NMR spectrometer equipped with a $3 \mathrm{~mm}$ inverse detection probe. NMR spectra were referenced to residual solvent $\mathrm{CDCl}_{3}$ signals $\left(\delta_{\mathrm{H}} 7.26\right.$ and $\delta_{\mathrm{C}} 77.16$ as internal standards). The NMR spectra were processed using MestReNova (Mnova 12.0, Mestrelab Research) or TopSpin 3.o (Bruker Biospin) software.

\section{Cyanobacterial Collection and Taxonomy.}

The marine cyanobacterium Symploca sp. (voucher specimen available from W. H. G. as collection no. ASS2oJul14-1) was found growing in 1.0 - 2.0 m of water at Fagaalu Park in American Samoa, U.S. The sample was hand collected in July 2014, preserved in a 1:1 2-propanol - seawater solution, and stored in the laboratory at $-20^{\circ} \mathrm{C}$ until extraction. Microscopic examination indicated that this collection was morphologically consistent with the genus Symploca sp.

\section{Extraction and Isolation}

The preserved cyanobacterium was filtered through cheesecloth, and the biomass (101.7 g dry wt) was extracted repeatedly by soaking in $500 \mathrm{~mL}$ of $2: 1 \mathrm{CH}_{2} \mathrm{Cl}_{2} / \mathrm{MeOH}$ with warming $\left(<30^{\circ} \mathrm{C}\right)$ for $30 \mathrm{~min}$ to afford $7.5 \mathrm{~g}$ of dried extract. A portion of the extract was fractionated by silica gel vacuum liquid chromatography (VLC) using a stepwise gradient solvent system of increasing polarity starting from $100 \%$ hexanes to $100 \% \mathrm{MeOH}$ [nine fractions, 100\% hexanes (Fr. A, $55.9 \mathrm{mg}$ ), 90\% hexanes/10\% EtOAc (Fr. B, 3.8 $\mathrm{mg}$ ), 80\% hexanes/20\% EtOAc (Fr. C $2.8 \mathrm{mg}$ ), 6o\% hexanes/40\% EtOAc (Fr. D, 37.0 mg), 40\% hexanes/6o\% EtOAc (Fr. E, 59.4 $\mathrm{mg}$ ), 20\% hexanes/80\% EtOAc (Fr. F, $62.7 \mathrm{mg}$ ), 100\% EtOAc (Fr. G, 21.3 mg), 75\% EtOAc/25\% MeOH (Fr. H, 98.9 mg) and 100\% $\mathrm{MeOH}$ (Fr. I, $434.9 \mathrm{mg}$ )]. Fr. $\mathrm{H}$ was suspended in $10 \% \mathrm{CH}_{3} \mathrm{CN} / 90 \% \mathrm{H} 2 \mathrm{O}$, subjected to chromatography on $\mathrm{C} 18$ solid-phase extraction (SPE) with a Strata $6 \mathrm{~mL}$ column and $1 \mathrm{~g}$ of C18-E $(55 \mu \mathrm{m}, 7 \mathrm{O} \AA$ ), and eluted sequentially with $30 \mathrm{~mL} 10 \% \mathrm{CH} 3 \mathrm{CN} / 90 \%$ $\mathrm{H}_{2} \mathrm{O}$ (Fr. H1, $\left.3.1 \mathrm{mg}\right), 30 \% \mathrm{CH}_{3} \mathrm{CN} / 70 \% \mathrm{H}_{2} \mathrm{O}$ (Fr. H2, $2.0 \mathrm{mg}$ ), 50\% $\mathrm{CH}_{3} \mathrm{CN} / 50 \% \mathrm{H}_{2} \mathrm{O}\left(\mathrm{Fr} . \mathrm{H}_{3}, 7.1 \mathrm{mg}\right), 70 \% \mathrm{CH} 3 \mathrm{CN} / 30 \% \mathrm{H} 2 \mathrm{O}(\mathrm{Fr}$. $\mathrm{H}_{4}, 17.5 \mathrm{mg}$ ), $100 \% \mathrm{CH}_{3} \mathrm{CN}$ (Fr. H5, $22.0 \mathrm{mg}$ ), 100\% MeOH (Fr. H6, $15.9 \mathrm{mg}$ ), and 100\% $\mathrm{CH}_{2} \mathrm{Cl}_{2}$ (Fr. $\mathrm{H}_{7}$, 10.0 mg). Fr. H4 was further purified by HPLC using a Kinetex $5 \mu \mathrm{m}$ RP $100 \AA$ A column $(10.00 \times 250 \mathrm{~mm})$ and isocratic elution using $97 \% \mathrm{ACN} / 3 \% \mathrm{H}_{2} \mathrm{O}$ at the flow rate of 3mL/min over $45 \mathrm{~min}$. This yielded six subfractions: Fr. H4-1 (2.o mg), Fr. H4-2 (2.o mg), Fr. H4-3 (3.4 mg), Fr. H4-4 (3.2 mg), Fr. H4-5 (1.8 mg), Fr. H4-6 (3.8 mg) at 10.0 - 16.0 min, 16.0 - 18.0 min, 18.0 - 20.0, 20.0-22.0, 22.0-24.0, 24.0-28.0, respectively. Fr. H4-6 was further purified by HPLC using a Kinetex $5 \mu \mathrm{m} \mathrm{RP} 100$ Å column $(10.00 \times 250 \mathrm{~mm})$ and gradient elution using $85 \% \mathrm{ACN} / 15 \% \mathrm{H}_{2} \mathrm{O}$ isocratic at the flow rate of $3 \mathrm{~mL} / \mathrm{min}$ for 5 minutes then ramping up to $100 \% \mathrm{ACN}$ in $15 \mathrm{minutes}$, then holding the gradient at $100 \% \mathrm{ACN}$ for another 5 minutes yielding compound 4 ( $0.3 \mathrm{mg}$ ) at $17.0 \mathrm{~min}$ and compound $\mathbf{1}(1.6 \mathrm{mg})$ at 21.3 min. Compounds 5-13 (samholides A-I) were isolated in microgram quantities as described before ${ }^{1}$ (Please see Figures S12-25 fore structure elucidation). Fr. I was dissolved in $40 \% \mathrm{CH}_{3} \mathrm{CN} / 60 \% \mathrm{H} 2 \mathrm{O}$, subjected to chromatography on $\mathrm{C}_{1} 8$ solid-phase extraction (SPE) with a Strata $6 \mathrm{~mL}$ column and $1 \mathrm{~g}$ of $\mathrm{C}_{1} 8-\mathrm{E}(55 \mu \mathrm{m}, 70 \mathrm{~A})$, and eluted sequentially with $30 \mathrm{~mL} 40 \% \mathrm{CH} 3 \mathrm{CN}_{3} 60 \% \mathrm{H} 2 \mathrm{O}(\mathrm{Fr}$. I1, $216.1 \mathrm{mg}$ ), 6o $\% \mathrm{CH}_{3} \mathrm{CN} / 40 \% \mathrm{H}_{2} \mathrm{O}$ (Fr. I2, $8.4 \mathrm{mg}$ ), 80\% $\mathrm{CH}_{3} \mathrm{CN} / 20 \% \mathrm{H}_{2} \mathrm{O}$ (Fr. I3, $7.2 \mathrm{mg}$ ), and 100\% CH3 CN (Fr. I4, $1.0 \mathrm{mg}$ ). Fr. I2 was further purified by HPLC using a Synergi $4 \mu \mathrm{m}$ Hydro-RP $80 \AA$ column (10.00 $\times 250 \mathrm{~mm})$ and isocratic elution using $97 \%$ $\mathrm{ACN} / 3 \% \mathrm{H}_{2} \mathrm{O}$ at the flow rate of $3 \mathrm{~mL} / \mathrm{min}$ over $45 \mathrm{~min}$. This yielded six subfractions: Fr. I2-1 (o.6 mg, contains compounds 5, 6), Fr. I2-2 (o.4 mg, contains compound 9, 10, 11), Fr.I2-3 (o.5 mg, contains compound 12), Fr. I2-4 (o.8 mg,), Fr. I2-5 (o.3 mg, contains compounds 7, 8), and Fr. I2-6 (o.1 mg) at $20.0-22.8 \mathrm{~min}, 22.8-24.6 \mathrm{~min}, 24.6-27.3 \mathrm{~min}, 27.3-28.8 \mathrm{~min}, 30.0-33.2 \mathrm{~min}$, and $34.4-37.5$ min, respectively. I3 was further purified by HPLC using a Synergi $4 \mu \mathrm{m}$ Hydro-RP 80 Å column (10.oo $\times 250 \mathrm{~mm})$ and isocratic elution using $97 \% \mathrm{ACN} / 3 \% \mathrm{H}_{2} \mathrm{O}$ at the flow rate of $3 \mathrm{~mL} / \mathrm{min}$ over $45 \mathrm{~min}$. This yielded six subfractions: Fr. I3-1 (<0.1 mg), Fr. I3-2 (1.2 mg), Fr.I3-3 (1.6 mg), Fr. I3-4 (o.8 mg), Fr. I3-5 (o.9 mg), and Fr. I2-6 (o.4 mg, contains compounds 7, 8, 13) at 5.0 - 19.0 $\min , 19.4-26.2 \mathrm{~min}, 26.2-29.0 \mathrm{~min}, 32.5-36.6 \mathrm{~min}, 36.6$ - $40.0 \mathrm{~min}$, and $42.0-44.5 \mathrm{~min}$, respectively.

\section{Stereochemistry}

All known swinholide-type compounds, irrespective of origin (sponge, cyanobacteria, algae, nudibranch), possess a highly analogous monomeric carbon skeleton as well as identical configurations at comparable chiral centers (Scheme 1); this latter aspect has been confirmed in two cases by X-ray crystallographic analysis ${ }^{2,3}$ as well as via total synthesis.4 Moreover, these swinholidetype metabolites are produced by highly similar trans AT polyketide synthase-type biosynthetic gene clusters.5,6 In the present case, close similarities between the ${ }^{1} \mathrm{H}$ and ${ }^{13} \mathrm{C}$ NMR chemical shifts and coupling constants of the protons at the chiral centers of compound 1 and those of previously reported swinholides, samholides, as well as the ankaraholides ${ }^{7}$, indicated that they have the same configurations at comparable centers (Tables S2, S3, and Scheme S1).

\section{Mass Spectrometry Data Analysis}

Ion trap: 
- Data conversion

- Data processing (classical molecular network).

Q-tof: The maXis q-tof mass spectrometry raw data (.d) were converted to the $\mathrm{m} / \mathrm{z}$ extensible markup language (.mzXML) with lock mass calibration applied using CompassXport (vers. 4.o.o.4) in Data Analysis 4.2 software (Bruker Daltonics, Bremen, Germany) running on Windows 10 PC.

The .mzXML files were converted to .mzML with MSConvert (vers. 3.0.19) ${ }^{8}$ and uploaded to GNPS. ${ }^{9}$ Feature detection/alignment was performed with the OpenMS-GNPS online workflow ${ }^{10}$, using OpenMS 2.4.o." Molecular networking analysis was performed with feature-based molecular networking analysis on GNPS $^{10}$. Spectral library search was performed against available public libraries and NIST17. The molecular networks were visualized in Cytoscape 3.7.1. ${ }^{12}$

\section{Data deposition}

The mass spectrometry data were deposited on MassIVE (accession numbers: for q-ToF: MSVoooo84453 and ion trap: XXX). The spectra annotated were deposited to the GNPS spectral library. The samholides derivatives annotations have the following accession number: CCMSLIBoooo5467782, CCMSLIBoooo5467781, CCMSLIBoooo5467783, CCMSLIBoooo5467784, CCMSLIBoooo5467786, CCMSLIBooo05467787, CCMSLIBooo05467788, CCMSLIBoooo5467790, CCMSLIBoooo5467792, CCMSLIBoooo5467791, CCMSLIBoooo5467794, CCMSLIBooo05467795, CCMSLIBoooo5467796, CCMSLIBoooo5467793, CCMSLIBoooo5467789, CCMSLIBoooo5467785, CCMSLIBoooo5463859, CCMSLIBoooo5436029-33, CCMSLIBoooo543855-59, and CCMSLIBoooo567781-96. The swinholides derivatives annotations have the following accession number: CCMSLIBoooo5463853, CCMSLIBoooo5466o97, CCMSLIBoooo5463854, CCMSLIBoooo5467778, CCMSLIBoooo5467780, CCMSLIBoooo5467779, CCMSLIBoooo5467777. The symplocolide derivative annotations have the following accession number: CCMSLIBoooo5467803, CCMSLIBoooo54678o, CCMSLIBoooo546780o. The job results and parameters for the classical molecular networking analysis of the ion trap can be accessed at the following address: https:/gnps.ucsd.edu/ProteoSAFe/status.jsp?task=1ade71d59c3941degdabo88oe7fd4fe6. The job results and parameters for the Networking Annotation Propagation analysis can be accessed at the following address https://gnps.ucsd.edu/ProteoSAFe/status.jsp?task=1c385eof685d45988d8240eb38aoiffo. The job results and parameters for the MolNetEnhancer analysis can be accessed at the following https:/gnps.ucsd.edu/ProteoSAFe/status.jsp?task=5a782437fc13449d9e62264fbcf61d29. For the q-ToF data, the jobs results and parameters for the OpenMS-GNPS workflow and feature-based molecular networking can be accessed at the following address: https://gnps.ucsd.edu/ProteoSAFe/status.jsp?task=3e402ac88ae144328b5d727176db4a4c, https://gnps.ucsd.edu/ProteoSAFe/status.jsp?task=26o8fof $48 f_{764}$ b57877c7b82ba4e595b, respectively.

\section{LCMSMS Analysis and Molecular Networking Generation}

The crude extract and fractions A - I were dissolved in $\mathrm{MeOH}$ and passed through a Bond Elut- $\mathrm{C} 18 \mathrm{OH}$ cartridge (Agilent Technologies, USA) that was prewashed with $3 \mathrm{~mL}$ of $\mathrm{CH}_{3} \mathrm{CN}$. Subsequently, the Bond Elut- $\mathrm{C}_{1} 8 \mathrm{OH}$ cartridge was washed with 3 $\mathrm{mL}$ of $\mathrm{CH}_{3} \mathrm{CN}$, the solvent dried under $\mathrm{N}_{2}$, and the residue dissolved in $\mathrm{MeOH}$. A o.ozo mL aliquot of each sample was injected and analyzed via LC - MS/MS on a ThermoFinnigan Surveyor Autosampler-Plus/LC-MS/MS/PDA-Plus system coupled to a Thermo Finnigan LCQ Advantage Max mass spectrometer with a gradient of $30-100 \% \mathrm{CH}_{3} \mathrm{CN}$ in water with o.1\% formic acid. The MS/MS spectra of the crude extract and nine fractions were used to generate a molecular network following previously described methodology and visualized using Cytoscape (www.cytoscape.org). Algorithms assumed a cosine threshold set at 0.7 and nodes were color-coded according to the fractions from VLC and HPLC isolation.

\section{High-resolution LC-MS/MS Analysis}

Samples were resuspended in $100 \%$ methanol. LC-MS/MS analysis was performed in an UltiMate 3000 UPLC system (Thermo Scientific) using a Kinetex $1.7 \mathrm{~mm} \mathrm{C18}$ reversed phase UHPLC column (50 X 2.1 mm) and Maxis Impact HD Q-TOF mass spectrometer (Bruker Daltonics) equipped with ESI source. The column was equilibrated with $5 \%$ solvent B (LC-MS grade acetonitrile, $0.1 \%$ formic acid) for $1 \mathrm{~min}$, followed by a linear gradient from $5 \%$ B to $100 \%$ B in 8 min, held at $100 \%$ B for 2 min. Then, $100 \%-5 \%$ B in $0.5 \mathrm{~min}$ and maintained at $5 \% \mathrm{~B}$ for $1 \mathrm{~min}$ at a flow rate of $0.5 \mathrm{~mL} / \mathrm{min}$ throughout the $12.5 \mathrm{~min}$ total run. MS spectra were acquired in positive ion mode in the range of $50-2000 \mathrm{~m} / \mathrm{z}$. A mixture of $10 \mathrm{mg} / \mathrm{mL}$ of each sulfamethazine, sulfamethizole, sulfachloropyridazine, sulfadimethoxine, amitriptyline, and coumarin was run before starting the run and after every 48 injections for quality control. An external calibration with ESI-Low Concentration Tuning Mix (m/z 118.086255; 322.048121; 622.028960; 922.009798; 1221.990637; 1521.971475; 1821.952313) (Agilent technologies) was performed prior to data collection. An internal calibrant Hexakis $\left({ }_{1} \mathrm{H}, 1 \mathrm{H}, 2 \mathrm{H}\right.$-perfluoroethoxy)phosphazene (CAS 186817-57-2, m/z 622.0290) was used throughout the runs. The capillary voltage of $4200 \mathrm{~V}$, nebulizer gas pressure (nitrogen) of 2 bar, ion source temperature of $200{ }^{\circ} \mathrm{C}$, dry gas flow of $9 \mathrm{~L} / \mathrm{min}$ source temperature, spectral rate of $3 \mathrm{~Hz}$ for MS1 and $10 \mathrm{~Hz}$ for MS2 was used. The parameters for acquisition of MS/MS fragmentation were set as it follows: the 5 most intense ions per MS1 were selected, MS/MS active exclusion parameter was enabled, set to 5 and to release after $30 \mathrm{~s}$, precursor ion was reconsidered for MS/MS if current intensity/previous intensity ratio $>\mathbf{2}$. Advanced stepping function was used to fragment ions according to the following table: 


\begin{tabular}{llll}
\hline Time & Collision RF & Transfer Time & Collision \\
\hline 0 & 450.0 & 70.0 & 125 \\
25 & 550.0 & 75.0 & 100 \\
50 & 800.0 & 90.0 & 100 \\
75 & 1100.0 & 95.0 & 75 \\
\hline
\end{tabular}

CID energies for MS/MS data acquisition were used according to the following table:

\begin{tabular}{ccccc}
\hline Type & Mass & Width & Collision & Charge State \\
\hline Base & 100.00 & 4.00 & 25.00 & 1 \\
Base & 100.00 & 4.00 & 20.00 & 2 \\
Base & 300.00 & 5.00 & 30.00 & 1 \\
Base & 300.00 & 5.00 & 25.00 & 2 \\
Base & 500.00 & 6.00 & 35.00 & 1 \\
Base & 500.00 & 6.00 & 30.00 & 2 \\
Base & 1000.00 & 7.00 & 45.00 & 1 \\
Base & 1000.00 & 7.00 & 40.00 & 2 \\
Base & 1500.00 & 8.00 & 50.00 & 1 \\
Base & 1500.00 & 8.00 & 50.00 & 2 \\
Base & 2000.00 & 10.00 & 60.00 & 1 \\
Base & 2000.00 & 10.00 & 60.00 & 2 \\
\hline
\end{tabular}

The mass of internal calibrant was excluded from the MS/MS list using a mass range of $m / z$ 621.5-623.0.

\section{Cytotoxicity assay}

Cytotoxicity to NCI-H46o human lung carcinoma cells was measured as cell viability using the 3-(4,5-dimethylthiazol-2-yl)-2,5diphenyltetrazolium bromide (MTT) reduction method. ${ }^{13}$ Cells were cultured in Roswell Park Memorial Institute (RPMI) 1640 medium containing L -glutamine (Mediatech, Manassas, VA) and supplemented with $1 \mathrm{nM}$ sodium py-ruvate, $100 \mu \mathrm{g} / \mathrm{mL}$ streptomycin, 100 units penicillin, $0.15 \%$ sodium bicarbonate, and $10 \%$ qualified fetal bovine serum $\left(\mathrm{FBS}, \mathrm{Gib}-\mathrm{co}^{\circledast}\right)$ at $37^{\circ} \mathrm{C}, 5^{\circ} \% \mathrm{CO}$ 2 . Cells were seeded in 96-well plates at 6660 cells/well in $180 \mu \mathrm{L}$. After $24 \mathrm{~h}$, positive (doxorubicin) and negative (RPMI) controls as well as either pure compound or fractions and subfractions were added to the wells. After $48 \mathrm{~h}$, the medium was removed by aspiration and cell viability determined by MTT staining. Optical density values were measured on a SpectraMax M2 microplate reader (Molecular Devices) at 570 and $630 \mathrm{~nm}$ and percent of death was calculated relative to the negative controls. Symplocolide A was dissolved in DMSO at $4.1 \mathrm{mg} / \mathrm{mL}(3 \mathrm{mM})$. Each dose - response curve used $20 \mathrm{uL}$ of this DMSO stock that were diluted with 180 $\mu \mathrm{L}$ of RPMI-1640 medium (without fetal bovine serum) to $300 \mathrm{uM}$ followed by nine serial $25 \mu \mathrm{L}: 54 \mu \mathrm{L}$ (half-log dilution) dilutions with RPMI-1640. Subsequently, $20 \mu \mathrm{L} /$ well of all 10 mixtures were added to cells in duplicate, resulting in a final maximal DMSO concentration of $1 \%$. Dose - response graphs gave an $\mathrm{IC}_{50}$ value of $1.66 \pm 0.94 \mu \mathrm{M}$ for symplocolide A and were received using GraphPad Prism version 8.o.o for Windows, GraphPad Software, San Diego, California USA, www.graphpad.com based on nine concentrations tested in duplicates and on two plates."

\section{SMART 2.0 analysis}

\section{Training of SMART 2.0}

The SMART system is a deep network that takes HSQC spectra as 10oxıo images, and maps them to a cluster space where nearby molecules have nearby structures [1]. The version of SMART we used (which we identify as SMART 2.0) was trained on 22,435 spectra that were computed from JEOL database tables, with 2,999 spectra used for early stopping. The spectra were mapped into a 180 -dimensional embedding space using a SqueezeNet ${ }^{14}$ that was pre-trained on Imagenet. ${ }^{15}$ The training used an image captioning approach, with the embedding from SqueezeNet used to train a Universal Transformer network ${ }^{16}$ to produce the corresponding SMILES strings (also taken from JEOL) from the embedding of the NMR spectrum. To prevent overfitting, we introduced an auxiliary learning objective to predict the Morgan Fingerprint ${ }^{17}$ (FP) of the SMILES string. This fingerprint represents the SMILES as a binary vector. Specifically, we compute FP with radius of 2 and use a 2048 bit representation.

Hence the training signal was based on cross-entropy loss on the SMILES strings, and the 2048 FP vector, backpropagated to train the embedding. In order to further populate the cluster space to improve dereplication, we also used the network to map an additional approximately 27,642 spectra that were computed from randomly chosen compounds in the Marinelit ((http://pubs.rsc.org/marinlit/), NPATLAS (https://www.npatlas.org/joomla/), and NPASS (http://bidd2.nus.edu.sg/NPASS/) databases into the 180-D space using the ACD Labs predictor (https://www.acdlabs.com/products/adh/nmr/nmr_pred/) to create the HSQC spectra. 


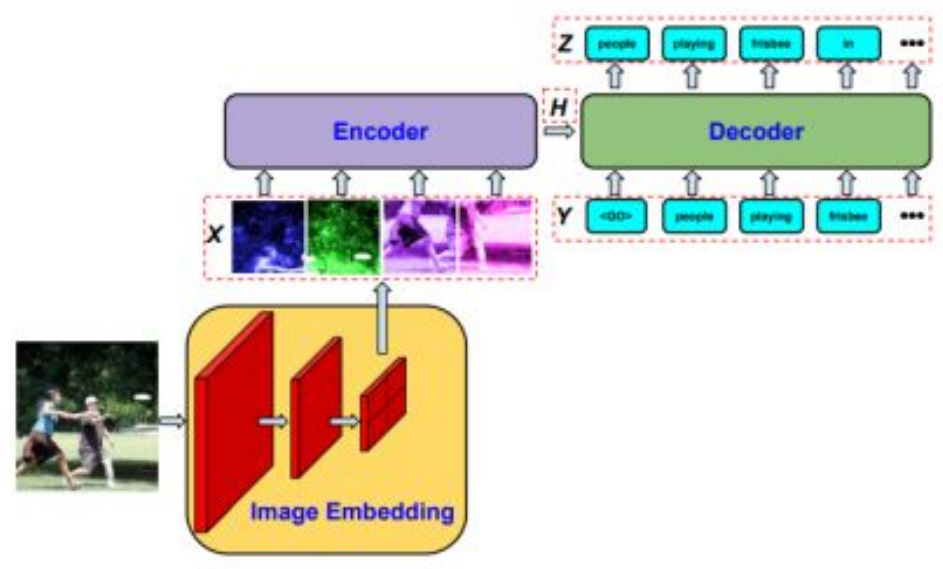

Model architecture

\section{Calculation of HSOC spectra}

Pereira et $\mathrm{al}^{18}$ pointed out that NP drug discovery process can only be exponentially improved with the inclusion of predicted spectral data using computational methods. In order to amplify the spectral data space, predicted spectra can be generated for known chemical spaces and for unknown chemical spaces exponentially amplified by automatic molecular structure generators. ACD/Spectrus Processor 2017.2.1 (File Version S7oS41, Build 99684, 21 Feb 2018) software was used for HSQC spectrum calculation of 27,642 spectra from randomly chosen compounds in the Marinelit ((http://pubs.rsc.org/marinlit/), NPATLAS (https://www.npatlas.org/joomla/), and NPASS (http://bidd2.nus.edu.sg/NPASS/) databases. All chemical structures were submitted by SMILES format and their HSQC spectrums were calculated by using corrected weighted average experimental algorithm. The calculation parameters were set as follows; Spectrometer frequency was 6oo $\mathrm{MHz}$ (fixed), spectrum size was 100 by 100 , spectrum bounds was single-dependent, line width was $3 \mathrm{~Hz}$ and solvent was $\mathrm{CDCl}_{3}$ as default and changed in specific investigations of solvent influence on SMART results (Figure S2). Peak lists were exported to text format and converted to CSV format for SMART application.

\section{How to use SMART 2.0}

The current version of SMART 2.0 as of $11 / 25 / 2019$ is trained on 2 D NMR spectra of 53,076 natural products and can be accessed via https://smart.ucsd.edu/classic. You can find detailed instructions on how to use SMART under https://mwang87.github.io/SMART_NMR_Documentation/. 
-... Z A $\mid$ Points: $53076 \mid$ Dimension: 180

(2)

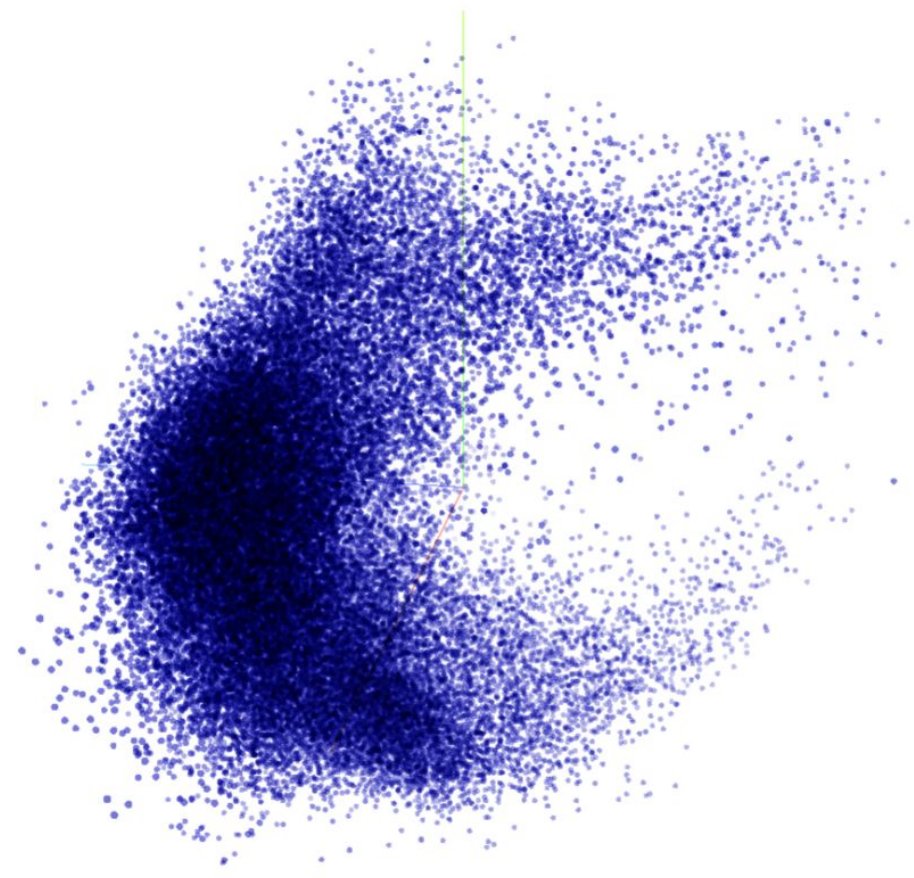

PCA analysis of HSQC spectra from 53,076 natural products. 


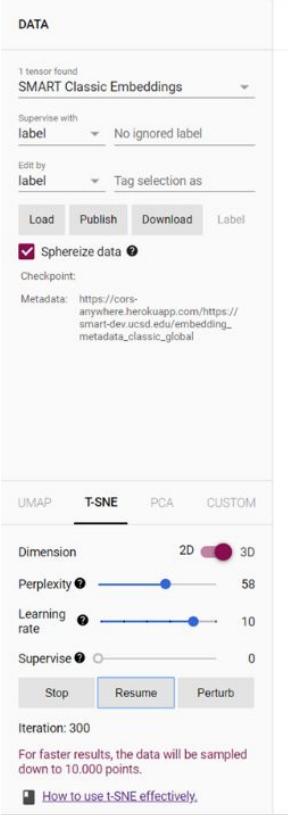

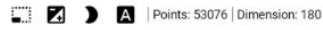

(2)
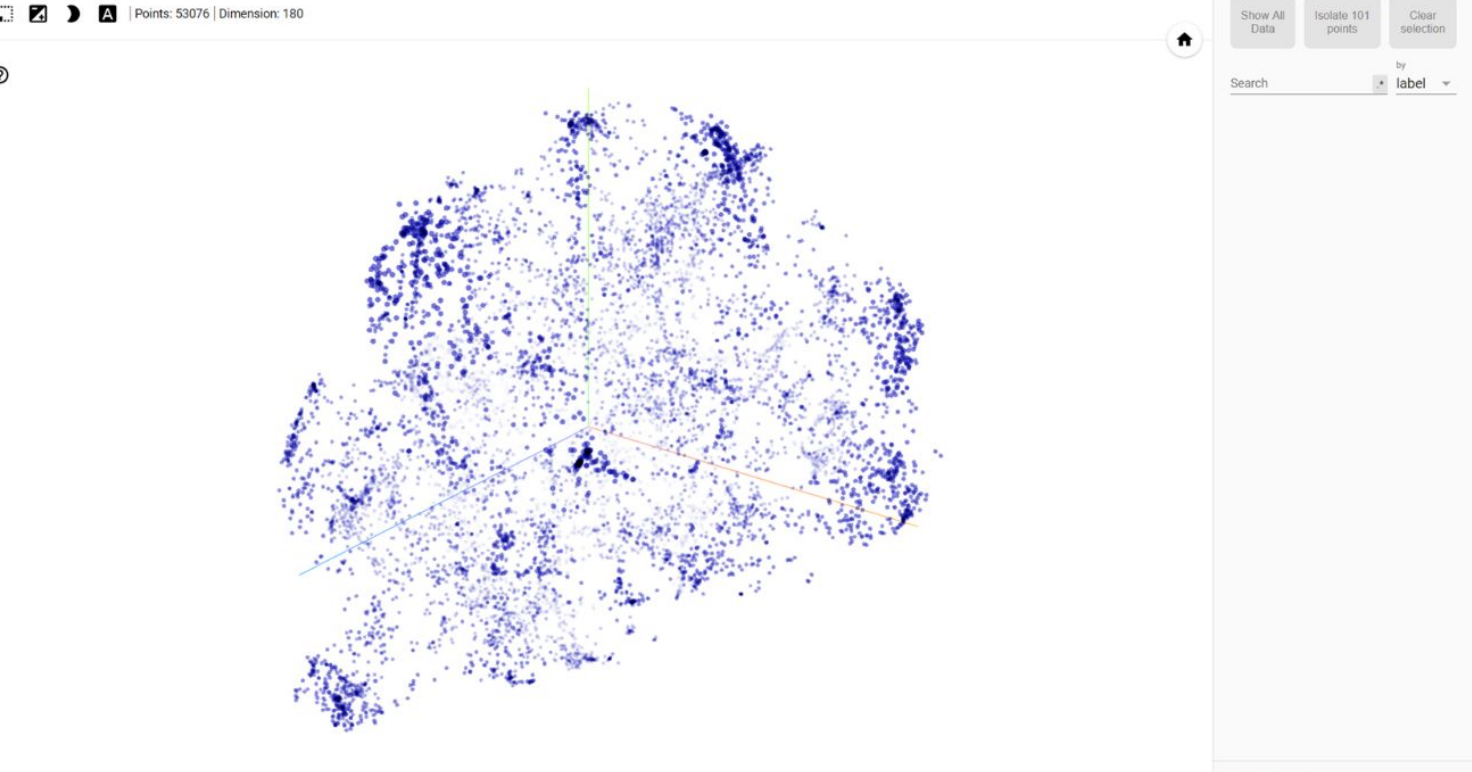

DATA
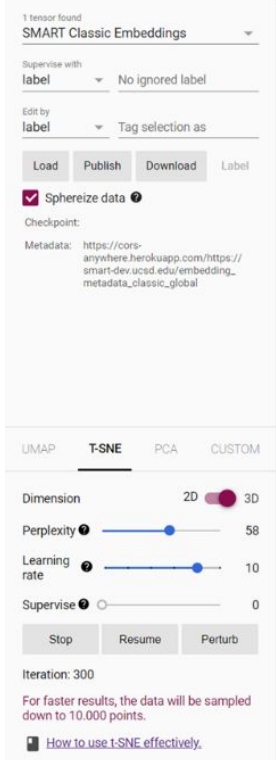

I D A $\mid$ Points: $53076 \mid$ Dimension $180 \mid$ Selected 101 points

(2)

t-SNE embedding of HSQC spectra from 53,076 natural products sampled down to 10,0oo nodes after 300 iterations (perplexity=58, learning rate=10). In the lower panel swinholide A and its 100 closest neighbors in the SMART DB are highlighted.

\section{User's Guide for SMART 2.0 Analysis}

Welcome to use the SMART 2.o to test your compound(s) @ SMART 2.o.

- The current version of SMART 2.0 as of 11/25/2019 consists of 2D NMR spectra from 53,076 natural products.

- $\quad$ Before you start, please unblock any pop-up blockers in your web browser for smart.ucsd.edu website.

- One SMART 2.0 analysis should take < 20 seconds (if still 'analyzing' double check your input).

- You can run multiple (up to 10) analyses at once.

- If your results are dissatisfying please try to process your data again manually (go back to How to process a raw HSQC spectrum to a NMR table and then delete noise and duplicate annotations, add peaks missed by auto-peak picking etc.) 


\section{Input Data Formatting}

Please prepare your NMR peak lists of each compound using Excel or preferably notepad/wordpad and save the NMR table of each compound as a comma-separated value (.csv) file. If you are using Microsoft Excel, this can be done by click "save as" and then choose the two file types. Please always place $1 \mathrm{H}$ data in the first column and their corresponding $13 \mathrm{C}$ data in the second column. The first row will be left for strings " $1 \mathrm{H}$ " and " $13 \mathrm{C}$ " as table head. Please strictly keep $1 \mathrm{H}$ shifts in 2 decimals and $13 \mathrm{C}$ shifts in a single decimal only. You can name the .csv file with any name that your operating system accepts.

$\begin{array}{ll}1 \mathrm{H} & 13 \mathrm{C} \\ 3.00 & 29.0 \\ 3.40 & 29.0 \\ 5.00 & 57.6 \\ 5.60 & 59.4 \\ 1.80 & 19.2 \\ 5.17 & 57.4 \\ 7.49 & 129.0 \\ 7.51 & 130.5 \\ 7.47 & 131.4\end{array}$

In the NMR table files, wherever there are diastereotopic protons on a methylene carbon (i.e., $\mathrm{CH} 2$ with two distinct proton shifts), please add a separate entry for both the carbon and proton:

$\begin{array}{ll}1 \mathrm{H} & 13 \mathrm{C} \\ 3.12 & 43.2 \\ 3.40 & 43.2\end{array}$

Supported Formats at SMART

SMART supports CSV formats for analysis. You can check your csv file by opening it with text editors such as wordpad or notepad. Your table should appear like this:

${ }_{1} \mathrm{H}, 13 \mathrm{C}$

$1.09,14.3$

$2.21,22.2$

$3.41,56.9$

$7.21,128.6$

$7 \cdot 29,123 \cdot 4$

\section{Submit CSV file for SMART Analysis}

After the .csv file is ready, simply upload it to the first window by drag\&drop (analysis automatically starts) or copy and paste the table and click analyze. You can submit multiple jobs at one time. Important: You have to allow pop-ups for https://smart.ucsd.edu/classic. If your analysis is still running after 20 seconds, pop-up blockers are the most likely reason why your analysis failed. The result will show up in the next webpage as images with chemical structures, compound names, similarity scores, and molecular weights. The Top 100 hits are ranked by similarity scores. You can download the result by clicking the "download results" icon and open it with Excel.

\section{Troubleshooting}

\section{Overview}

This section addresses some common issues with the analysis workflows at SMART. If you run into any of these common issues, hopefully this page will give you actionable steps to address them. If this page cannot help you, please refer to the forum to help answer your questions. 


\section{SMART Analysis}

Failed Job

1. If your analysis is still running after 20 seconds, pop-up blockers are the most likely reason why your analysis failed. You have to allow pop-ups for https://smart.ucsd.edu/classic.

2. The file format input is incorrect. Please make sure it is a supported file format for SMART (see Input Data Formatting) Downloaded Results file cannot be opened

Yes you can :) You can open the downloaded results with any text editor (Excel, Notepad, Wordpad). Just make a right-click on the file, click on 'open with' and then choose one of the former mentioned programs.

\section{Results Incorrect}

The SMART is still at its early stage of development. It will become more and more accurate the more spectra you contribute to the Moliverse (see Contribute to SMART). - If your results show strange suggestions and all of the have a cosine score of 1.0 you probably have switched the columns. Please be sure that your first column has proton shifts $(\mathrm{iH})$ and your second column has carbon shifts $\left({ }_{13} \mathrm{C}\right)$, where each ${ }_{1} \mathrm{H}-13 \mathrm{C}$ pair is separated by a comma.

Visualize your result(s) in the $3 \mathrm{D}$ cluster space (Moliverse)

Please click the "Embed in the Moliverse" bar in the upper right side of the result page. Wait a while for the Embedding Projector to launch. To find your query molecule, please type "query" in the search bar in the upper right side of the Embedding Projector Webpage. Naturally, your query compound as well as its 100 closest analogues will light up in unique colors (decreasing cosine similarity will be displayed by purple-red-orange-yellow).

- To better observe the clustering effect, please click UMAP or T-SNE tabs in the lower left window. The clusters will organize themselves in real-time, clustering more similar compounds in close proximity and more dissimilar compounds apart from each other.

- Further you can access and enjoy the whole 'Moliverse' under https://smart.ucsd.edu/classic (click on 'Explore Moliverse')

Visualize the $3 \mathrm{D}$ cluster space (Moliverse) with a VR headset

Please click here to watch the VR movie. The VR software will be delivered soon!

Contact

If you have any questions about how to use SMART please first ask the community at the SMART forum located here: forum. We will respond you as soon as we can.

Please find an updated version of the documentation at: https://mwang87.github.io/SMART_NMR_Documentation/

\section{Instructions for submitting NMR data to the Moliverse (the training set for SMART)}

Please read all of the instructions below before you fill in the data collection files.

\section{Definitions}

Folder $=$ a folder on your computer for organizing files; File $=$ an excel file in.$x l s x$ or .csv format; Sheet $=$ a sheet within an excel file; Training database $=$ the database used to train the AI; Test dataset $=$ the inquiry dataset awaiting the AI to generate structural hypothesis

\section{Submitting structures to the Moliverse library}

Thank you for contributing to the SMART training database (AKA the Moliverse Library). For this submission, we ask you to fill out some information in the metadata file (only one metadata file for all compounds being submitted), and a second file with the tabulated NMR data as described below (the NMR data for each compound in its own file).

Connection between the two files will be by a 6 digit code number. The starting letter/number combination for your submitted compounds will be assigned by the SMART operators, and then you should increment up for each ensuing compound by a value of 1 (e.g. AAAoor is the assigned code, the next compound in your series is AAAooz, and so forth). Although compound replicates need to be avoided, please don't worry as the SMART can cope with some replicates. You can continue adding more entries on the pre-existing metadata file with the already given code pattern. 
In the Metadata file and the NMR Tables, only the items with an orange colored background are required; the green fields are desirable and will help us with developing more accurate and informative future versions of SMART. If for any compound, a specific data entry is not available, please leave it as blank.

As noted above, in the Metadata file, only the SMILES entry is mandatory. Only when the isomeric SMILES is not available for a compound, canonical SMILES can be entered instead. The SMILES can be found on the PubChem website for majority of published compounds. If you cannot fine the SMILES on PubChem, the $3 \mathrm{D}$ structure in ChemDraw can be converted to SMILES by the program.

\section{Convert Structure to SMILES}

To convert a ChemDraw structure to SMILES, you must: 1) Select the structure using the selection tool; 2) From the Edit menu, point to Copy As, and then choose SMILES; 3) Paste the string in the target cell of the excel metadata file sheet.

\section{Minimum information for NMR tables}

For compiling the NMR tables, please follow the examples in "NMR Tables" file in this same folder as the format for your data. NOTE: proton shifts should be rounded to exactly 2 decimal digits, and ${ }_{13} \mathrm{C}$ shifts should be rounded to exactly a single decimal digit. For the NMR tables, the atom numbering is not needed, but just be certain that the proton shift matches the carbon shift in every row of the table.

In the NMR table files, wherever there are diastereotopic protons on a methylene carbon (i.e., $\mathrm{CH} 2$ with two distinct proton shifts), please add a separate entry for both the carbon and proton:

\begin{tabular}{lll}
\multicolumn{2}{c}{ Column A } & Column B \\
${ }_{13} \mathrm{C}$ & $1 \mathrm{H}$ & Carbon type (optional) \\
43.2 & 3.12 & $\mathrm{CH} 2$ \\
43.2 & 3.40 & $\mathrm{CH} 2$
\end{tabular}

In the NMR table files, please put ${ }_{13} \mathrm{C}$ shifts in Column A of the sheet and proton shifts in Column B of the sheet, as shown above. If you have any questions, please contact Chen Zhang chzo23@ucsd.edu or Raphael Reher (rreher@ucsd.edu).

\section{Mixture Analysis}

Peaks were picked automatically with the auto-peak picking function of MestreNova at a high threshold. The threshold was then reduced and additional weak peaks were added. Noise, artifacts and two bond correlations were removed. Peaks picked on individual lines in multiplets were combined to a single peak. 

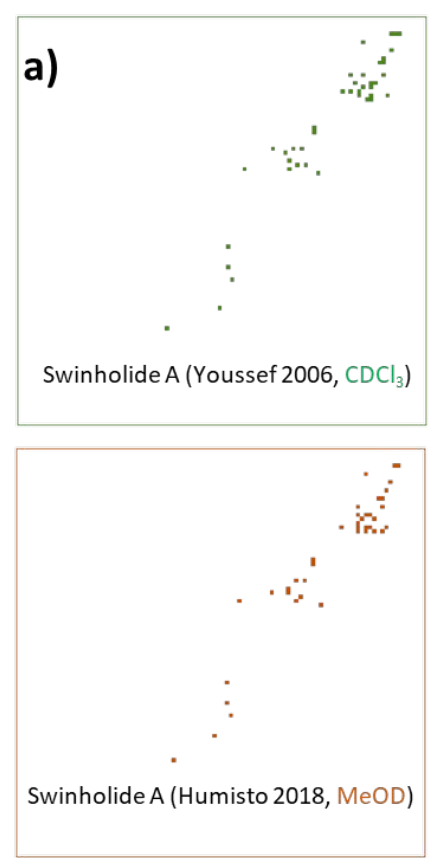

c)

\begin{tabular}{|l|r|}
\hline Name & Score \\
\hline swinholide A & 0.992 \\
\hline swinholide I & 0.977 \\
\hline hurghadolide A & 0.969 \\
\hline ankaraholide A & 0.933 \\
\hline halichoblelide B & 0.927 \\
\hline Cytovaricin B & 0.913 \\
\hline swinholide A(Calcd_CDCI3) & 0.912 \\
\hline hurghadolide A(Calcd_CDCI3) & 0.905 \\
\hline neomaclafungin D & 0.903 \\
\hline ushikulide A & 0.9 \\
\hline swinholide K(Calcd_CDCl3) & 0.898 \\
\hline isoswinholide B(Calcd_CDCl3) & 0.895 \\
\hline neaumycin & 0.893 \\
\hline neomaclafungin B & 0.893 \\
\hline neomaclafungin C & 0.887 \\
\hline neomaclafungin H & 0.884 \\
\hline azalomycin F5a & 0.88 \\
\hline azalomycin F4a 2-ethylpentyl & 0.873 \\
\hline 6-Hydroxyscytophycin B(Calcc & 0.873 \\
\hline luminaolide & 0.87 \\
\hline
\end{tabular}

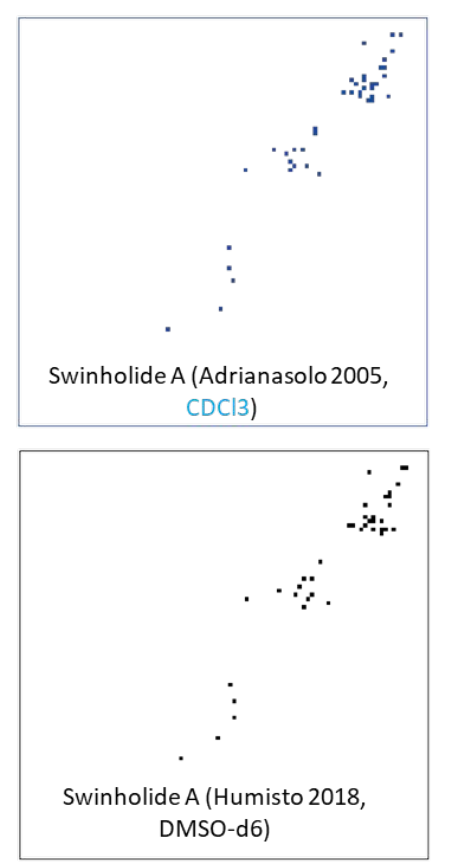

b)

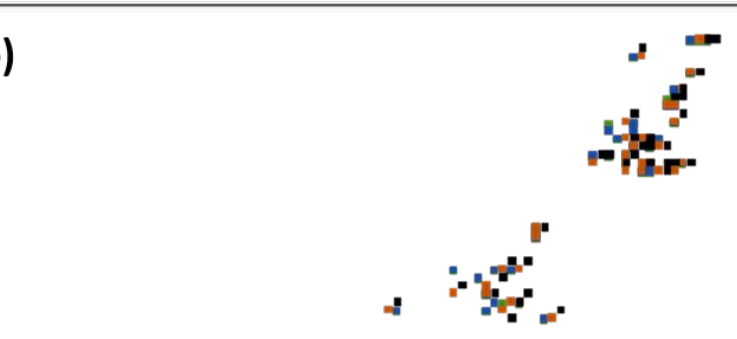

\begin{tabular}{|l|r|}
\hline Name & Score \\
\hline swinholide I & 0.98 \\
\hline hurghadolide A & 0.967 \\
\hline swinholide A & 0.965 \\
\hline hurghadolide A(Calcd_CDCl3) & 0.933 \\
\hline ankaraholide A & 0.922 \\
\hline swinholide A(Calcd_CDCl3) & 0.921 \\
\hline swinholide K(Calcd_CDCl3) & 0.92 \\
\hline azalomycin F5a & 0.898 \\
\hline neomaclafungin D & 0.898 \\
\hline isoswinholide B(Calcd_CDCl3) & 0.897 \\
\hline halichoblelide B & 0.891 \\
\hline neomaclafungin B & 0.889 \\
\hline azalomycin F4a 2-ethylpentyl est & 0.888 \\
\hline azalomycin F5a 2-ethylpentyl est & 0.881 \\
\hline azalomycin F4a & 0.879 \\
\hline neaumycin & 0.877 \\
\hline Cytovaricin B & 0.875 \\
\hline ushikulide A & 0.875 \\
\hline neomaclafungin H & 0.869 \\
\hline neomaclafungin F & 0.863 \\
\hline
\end{tabular}

\begin{tabular}{|l|r|}
\hline Name & Score \\
\hline swinholide I & 0.939 \\
\hline swinholide A(Calcd_CDCl3 & 0.932 \\
\hline swinholide A & 0.93 \\
\hline hurghadolide A & 0.926 \\
\hline ushikulide A & 0.9 \\
\hline isoswinholide B(Calcd_CDC & 0.895 \\
\hline neomaclafungin D & 0.89 \\
\hline halichoblelide B & 0.89 \\
\hline swinholide K(Calcd_CDCl3) & 0.884 \\
\hline hurghadolide A(Calcd_CDCl & 0.88 \\
\hline neomaclafungin B & 0.88 \\
\hline ankaraholide A & 0.874 \\
\hline azalomycin F5a & 0.867 \\
\hline Cytovaricin B & 0.859 \\
\hline azalomycin F4a 2-ethylpen & 0.855 \\
\hline ushikulide B & 0.855 \\
\hline neomaclafungin H & 0.854 \\
\hline neomaclafungin F & 0.851 \\
\hline azalomycin F4a & 0.851 \\
\hline neomaclafungin E & 0.849 \\
\hline
\end{tabular}

Figure S1: Influence of different researchers and NMR solvents on SMART 2.0 results. a) Single and b) overlayed experimental HSQC spectra of swinholide A recorded in $\mathrm{CDCl}_{3}$ by Youssef et $\mathrm{al}^{19}$ (green, upper left) and Andrianasolo et al ${ }^{7}$ (blue, upper right), in MeOD (marron, lower left) and in DMSO-d6 (black, lower right) by Humisto et al. ${ }^{6} \mathrm{c}$ ) Top2o results of SMART 2.0 analysis ordered by $180 \mathrm{D}$ cosine similarity score. 

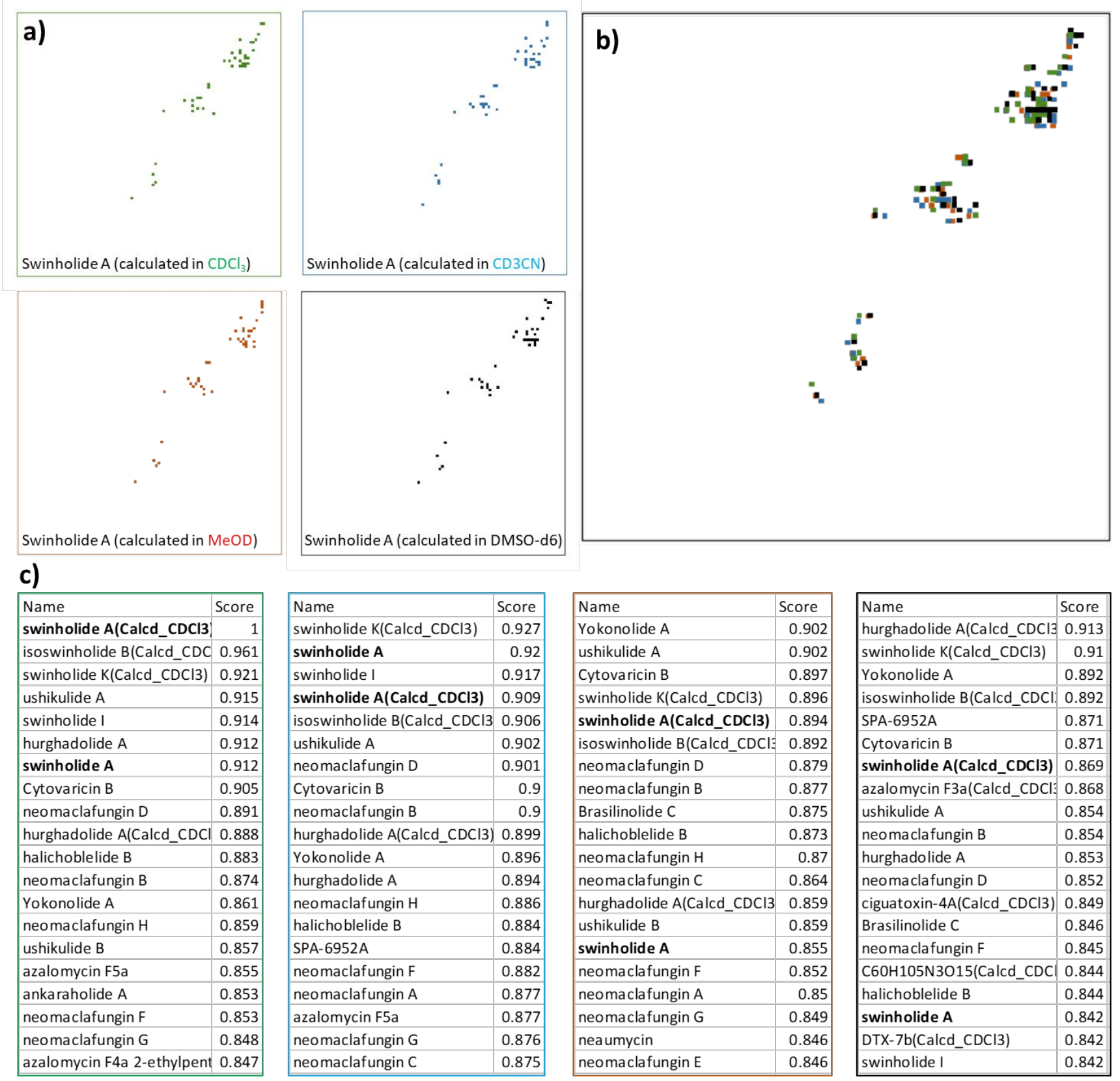

\begin{tabular}{|l|r|}
\hline Name & Score \\
\hline swinholide K(Calcd_CDCl3) & 0.927 \\
\hline swinholide A & 0.92 \\
\hline swinholide I & 0.917 \\
\hline swinholide A(Calcd_CDCl3) & 0.909 \\
\hline isoswinholide B(Calcd_CDCl3 & 0.906 \\
\hline ushikulide A & 0.902 \\
\hline neomaclafungin D & 0.901 \\
\hline Cytovaricin B & 0.9 \\
\hline neomaclafungin B & 0.9 \\
\hline hurghadolide A(Calcd_CDCI3) & 0.899 \\
\hline Yokonolide A & 0.896 \\
\hline hurghadolide A & 0.894 \\
\hline neomaclafungin H & 0.886 \\
\hline halichoblelide B & 0.884 \\
\hline SPA-6952A & 0.884 \\
\hline neomaclafungin F & 0.882 \\
\hline neomaclafungin A & 0.877 \\
\hline azalomycin F5a & 0.877 \\
\hline neomaclafungin G & 0.876 \\
\hline neomaclafungin C & 0.875 \\
\hline \hline
\end{tabular}

\begin{tabular}{|l|r|}
\hline Name & Score \\
\hline Yokonolide A & 0.902 \\
\hline ushikulide A & 0.902 \\
\hline Cytovaricin B & 0.897 \\
\hline swinholide K(Calcd_CDCl3) & 0.896 \\
\hline swinholide A(Calcd_CDCI3) & 0.894 \\
\hline isoswinholide B(Calcd_CDCl & 0.892 \\
\hline neomaclafungin D & 0.879 \\
\hline neomaclafungin B & 0.877 \\
\hline Brasilinolide C & 0.875 \\
\hline halichoblelide B & 0.873 \\
\hline neomaclafungin H & 0.87 \\
\hline neomaclafungin C & 0.864 \\
\hline hurghadolide A(Calcd_CDCl3 & 0.859 \\
\hline ushikulide B & 0.859 \\
\hline swinholide A & 0.855 \\
\hline neomaclafungin F & 0.852 \\
\hline neomaclafungin A & 0.85 \\
\hline neomaclafungin G & 0.849 \\
\hline neaumycin & 0.846 \\
\hline neomaclafungin E & 0.846 \\
\hline
\end{tabular}

\begin{tabular}{|l|r|}
\hline Name & Score \\
\hline hurghadolide A(Calcd_CDCl3 & 0.913 \\
\hline swinholide K(Calcd_CDCl3) & 0.91 \\
\hline Yokonolide A & 0.892 \\
\hline isoswinholide B(Calcd_CDCl: & 0.892 \\
\hline SPA-6952A & 0.871 \\
\hline Cytovaricin B & 0.871 \\
\hline swinholide A(Calcd_CDCl3) & 0.869 \\
\hline azalomycin F3a(Calcd_CDCl: & 0.868 \\
\hline ushikulide A & 0.854 \\
\hline neomaclafungin B & 0.854 \\
\hline hurghadolide A & 0.853 \\
\hline neomaclafungin D & 0.852 \\
\hline ciguatoxin-4A(Calcd_CDCl3) & 0.849 \\
\hline Brasilinolide C & 0.846 \\
\hline neomaclafungin F & 0.845 \\
\hline C60H105N3O15(Calcd_CDCl & 0.844 \\
\hline halichoblelide B & 0.844 \\
\hline swinholide A & 0.842 \\
\hline DTX-7b(Calcd_CDCl3) & 0.842 \\
\hline swinholide I & 0.842 \\
\hline
\end{tabular}

Figure S2: Influence of NMR solvents for predicted (calculated with ACD/Labs predictor) HSQC spectra on SMART 2.o results. a) Single and $\mathrm{b}$ ) overlayed predicted $\mathrm{HSQC}$ spectra of swinholide A calculated for $\mathrm{CDCl}_{3}$ (green, upper left), $\mathrm{CD}_{3} \mathrm{CN}$ (blue, upper right), ) MeOD (marron, lower left) and DMSO-d6 (black, lower right) c) Top2o results of SMART 2.0 analysis ordered by $180 \mathrm{oD}$ cosine similarity score. 


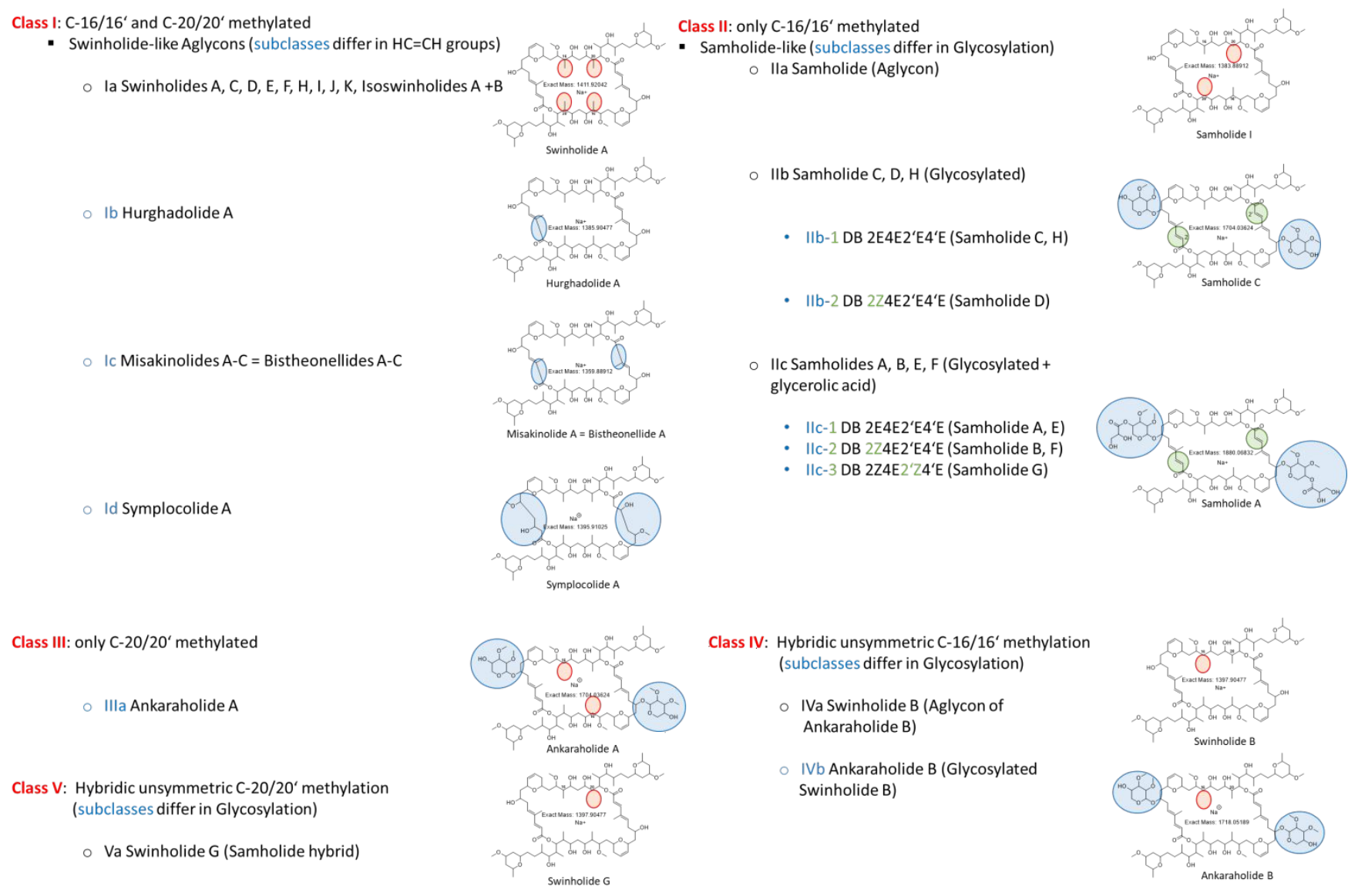

Scheme S1. New chemically/biosynthetically sound classification scheme of swinholide-like (40-44-membered) dilactone macrolides into five different classes (C-16/C-16' and C-20/C-20' methylation pattern) and 8 subclasses (lacking DB, different DB sterochemisrty, glycosylation). 

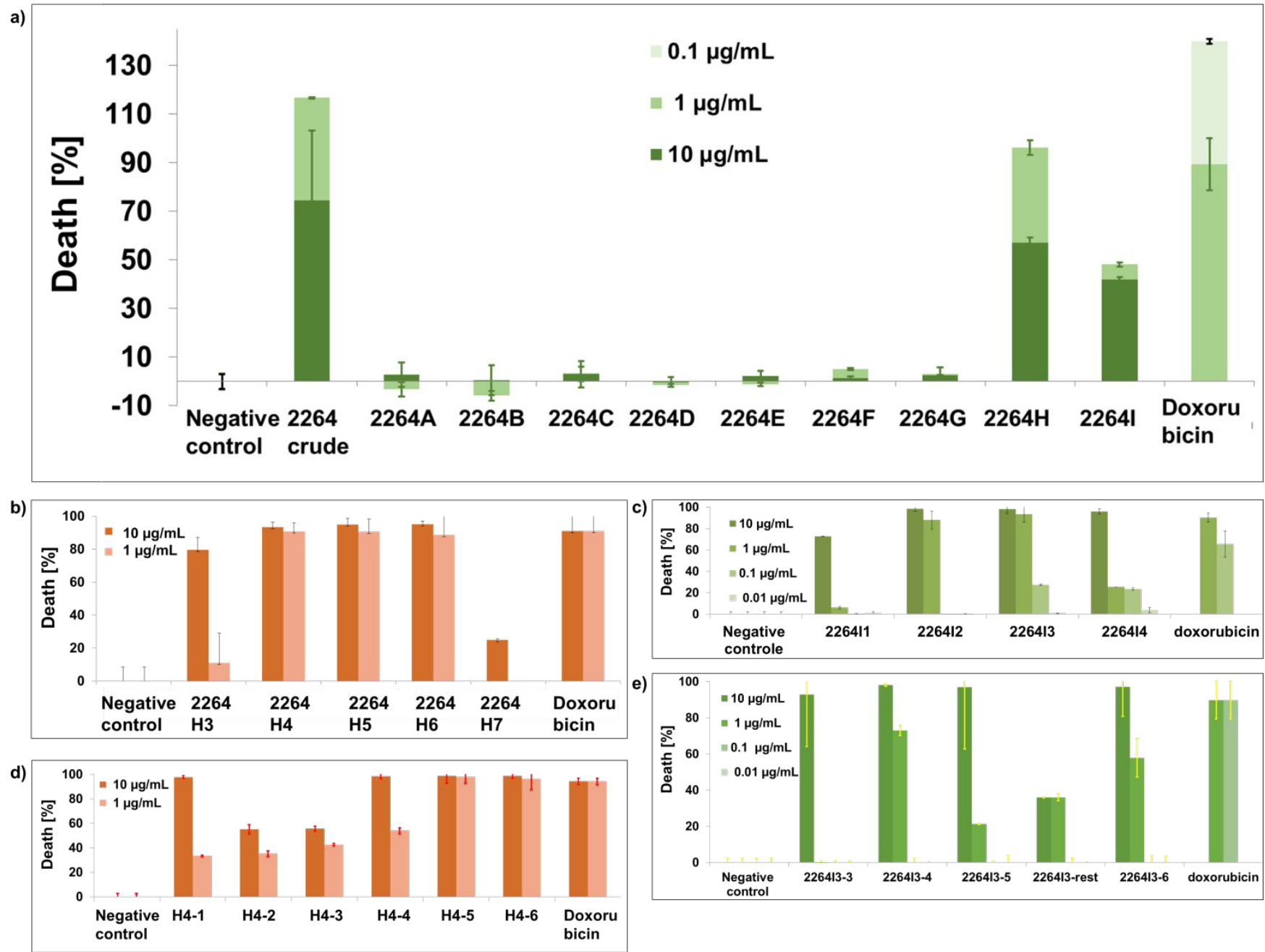

Figure S3: Cytotoxicity assay results against NCI H-46o lung cancer cells of a) 2264 crude (2264 = collection code), nine vacuum liquid chromatography (VLC) fractions (A-I) and further purification stages of the most active VLC fractions $\mathrm{H}+\mathrm{I}$ from the investigated Symploca species; b) and c) activity of VLC sub-fractions from H or I, respectively, were purified via C-18 solid phase extraction with an acetonitrile/water gradient of decreasing polarity; active fractions (d) $\mathrm{H}_{4}$ (symplocolide-containing) and I3, (e), respectively (samholide-containing) were further purified by HPLC (for details on the purification process see supporting information page $\left.\mathrm{S}_{3}\right)$. Negative control is media with $1 \%$ DMSO alone, positive control is doxorubicin $(1 \mu \mathrm{g} / \mathrm{mL} \mathrm{and} \mathrm{o.1} \mu \mathrm{g} / \mathrm{mL})$ in $1 \%$ DMSO. Percent cell death values are mean values of duplicate measurements for each concentration. The error bars represent range of these two measurements. 
a)
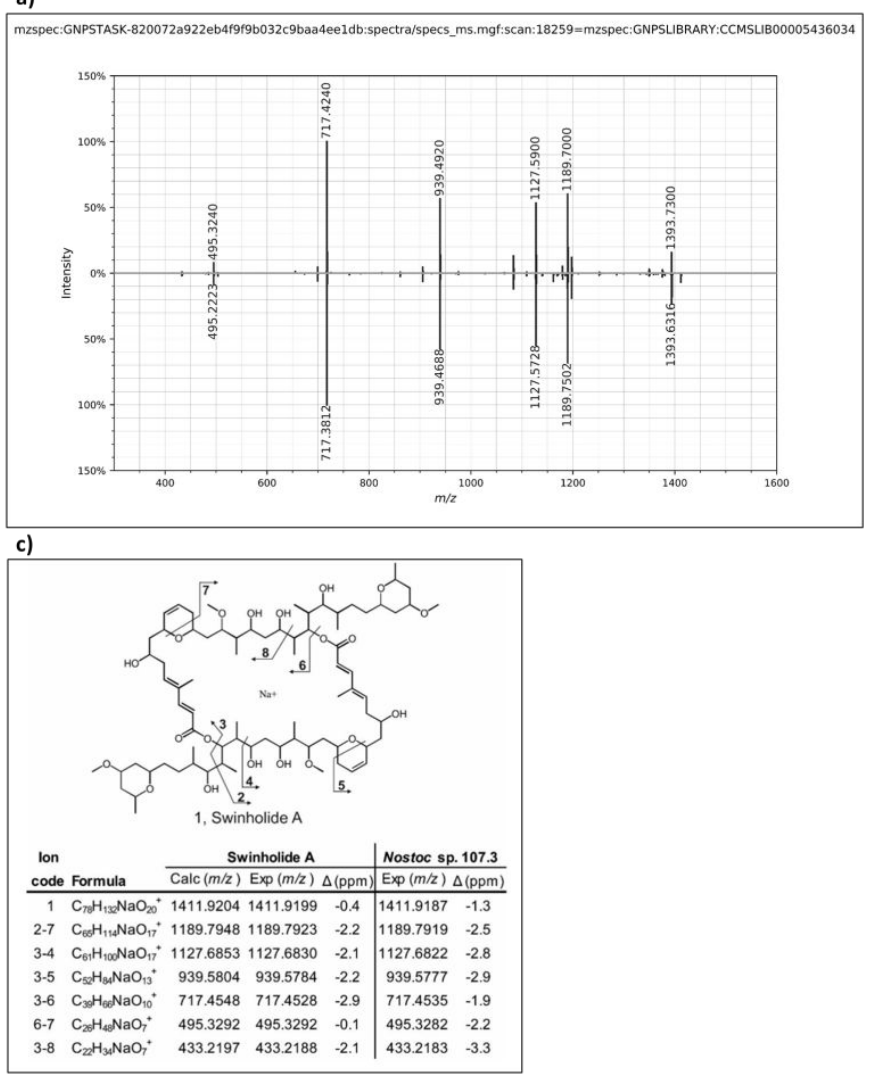

b)

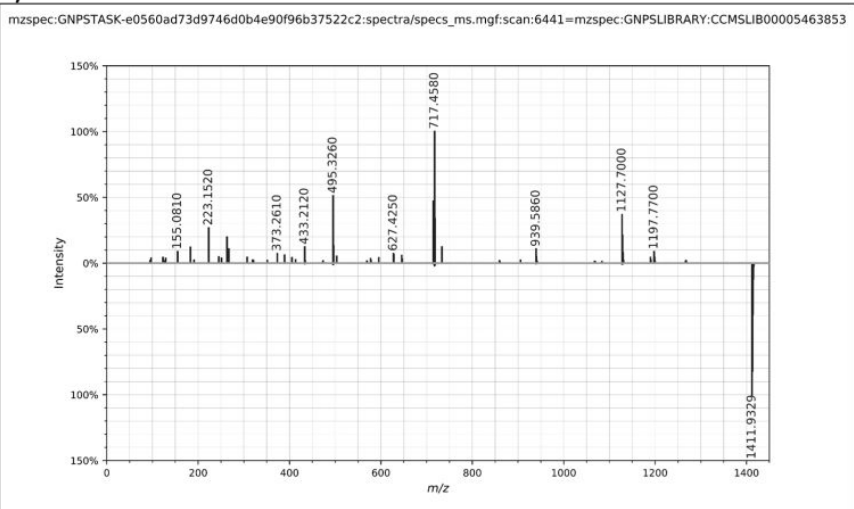

d)

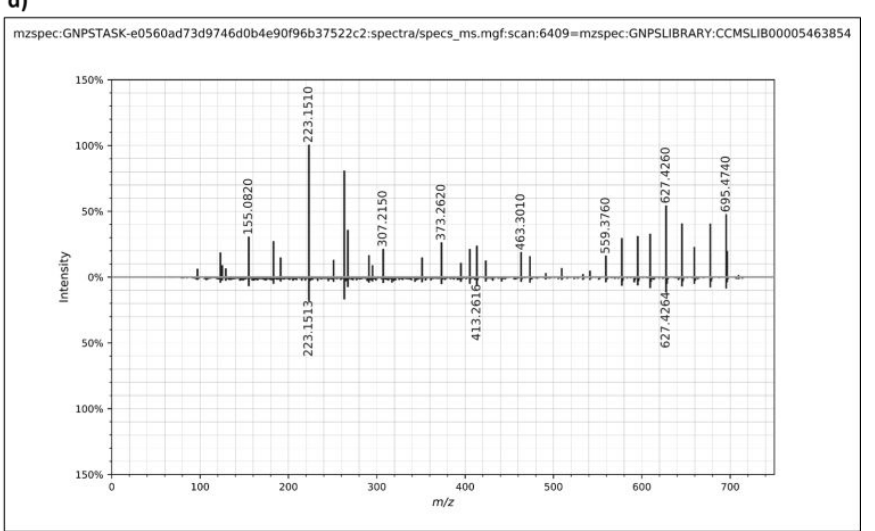

Figure S4: (a) Mirror plot of ESI iontrap MS2 spectrum and GNPS library spectrum [M+Na $]^{+}$of compound 4 (swinholide A) and (b) mirror plot of high-resolution ESI q-ToF MS2 spectrum of $4[\mathrm{M}+\mathrm{Na}]^{+}$from this study and GNPS library spectrum $[\mathrm{M}+\mathrm{Na}]^{+}$of 4. (c) Fragmentation scheme of $\mathbf{4}$ from Humisto et al. d) Mirror plot of high-resolution ESI q-ToF MS² spectrum (top) and GNPS library spectrum $[\mathrm{M}+\mathrm{H}]^{+}$of $\mathbf{4}$ (bottom). $\mathrm{m} / \mathrm{z}$ range was set to fingerprint region o-75o Da. 


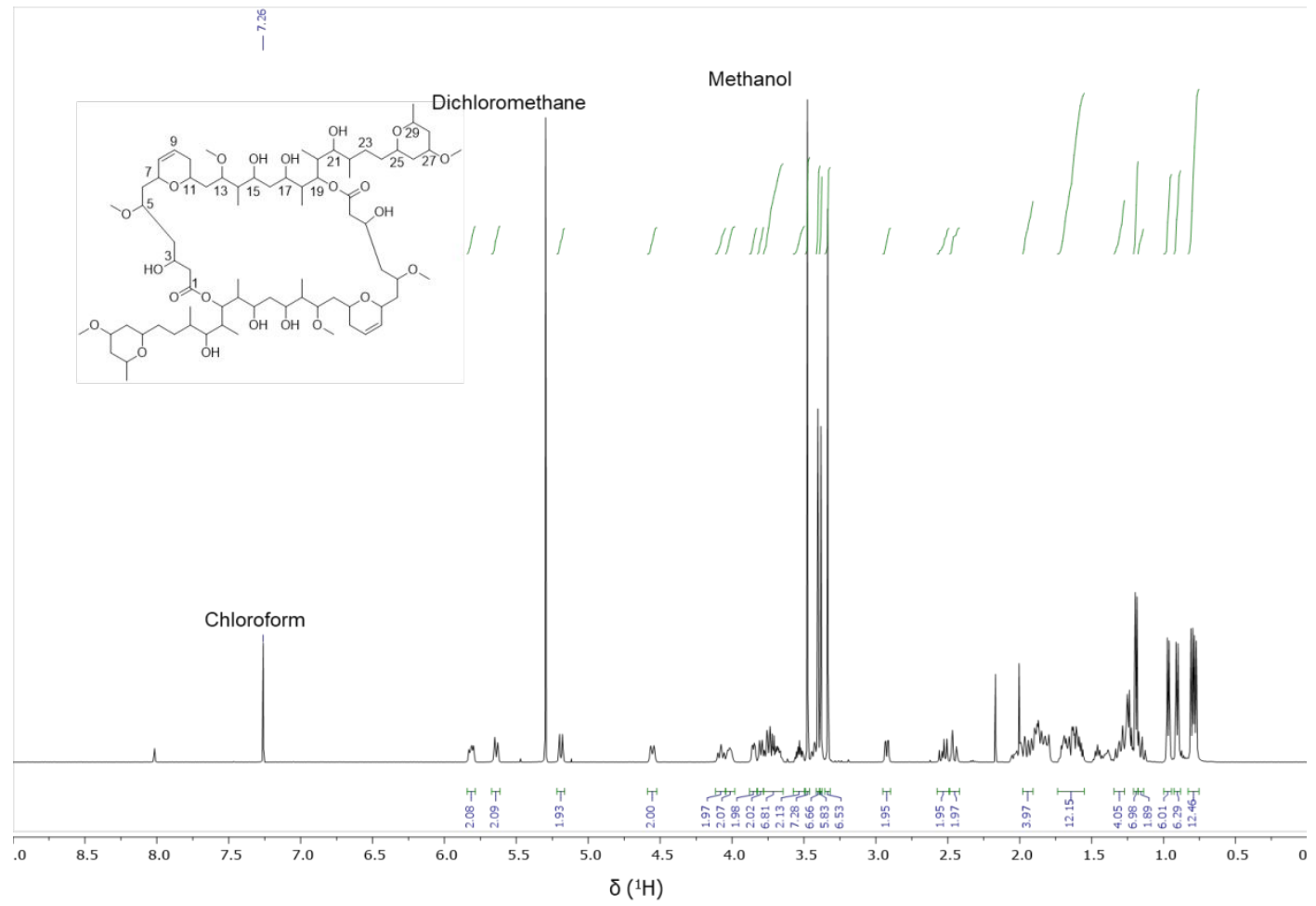

Figure $\mathrm{S}_{5}:{ }^{1} \mathrm{H}$ NMR spectrum (50o $\mathrm{MHz}, \mathrm{CDCl}_{3}$ ) of compound $\mathbf{1}$ (symplocolide $\left.\mathrm{A}\right)$.

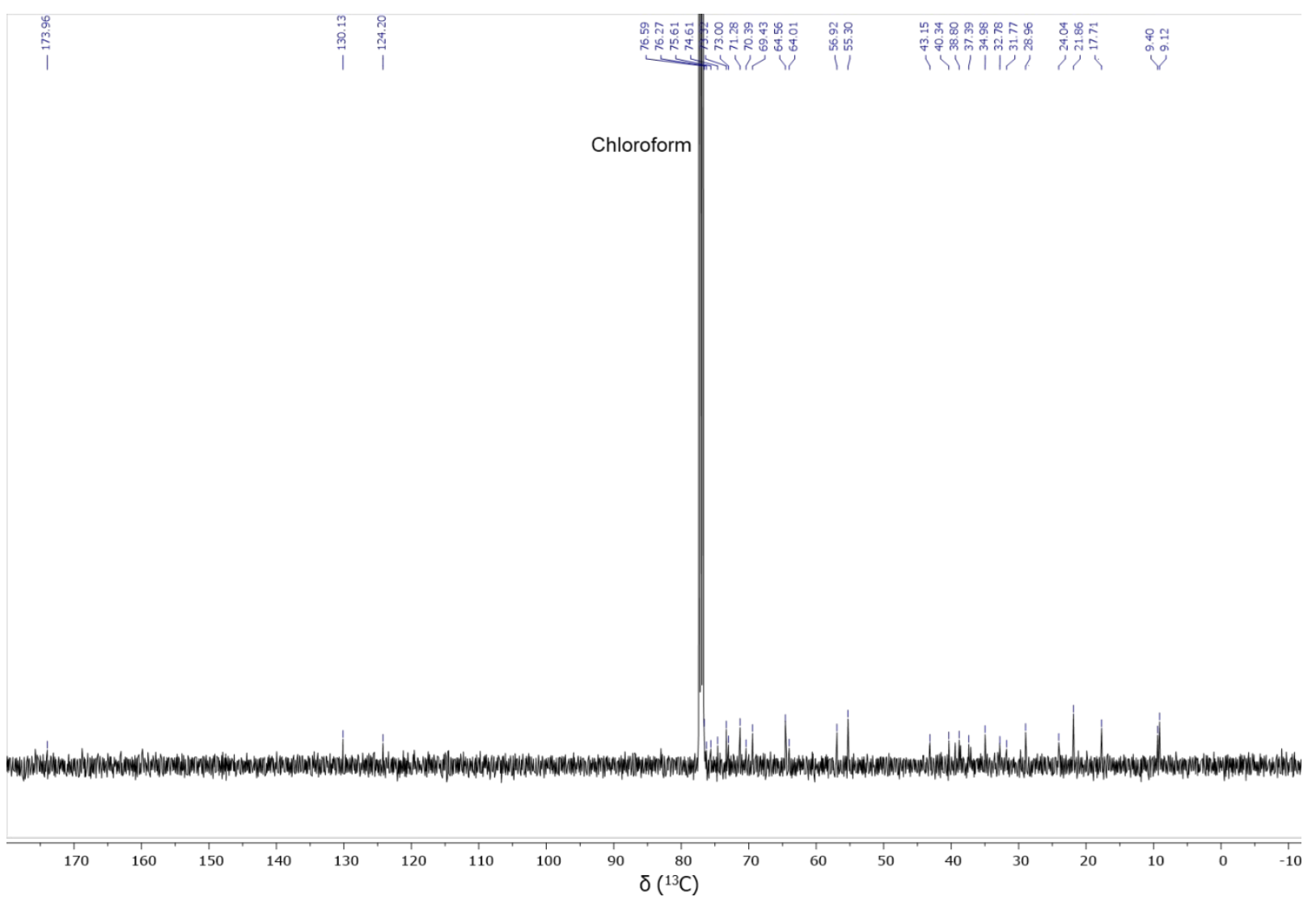

Figure S6: ${ }^{13} \mathrm{C}$ NMR spectrum (125 $\mathrm{MHz}^{\mathrm{CDCl}}{ }_{3}$ ) of compound $\mathbf{1}$ (symplocolide A). 


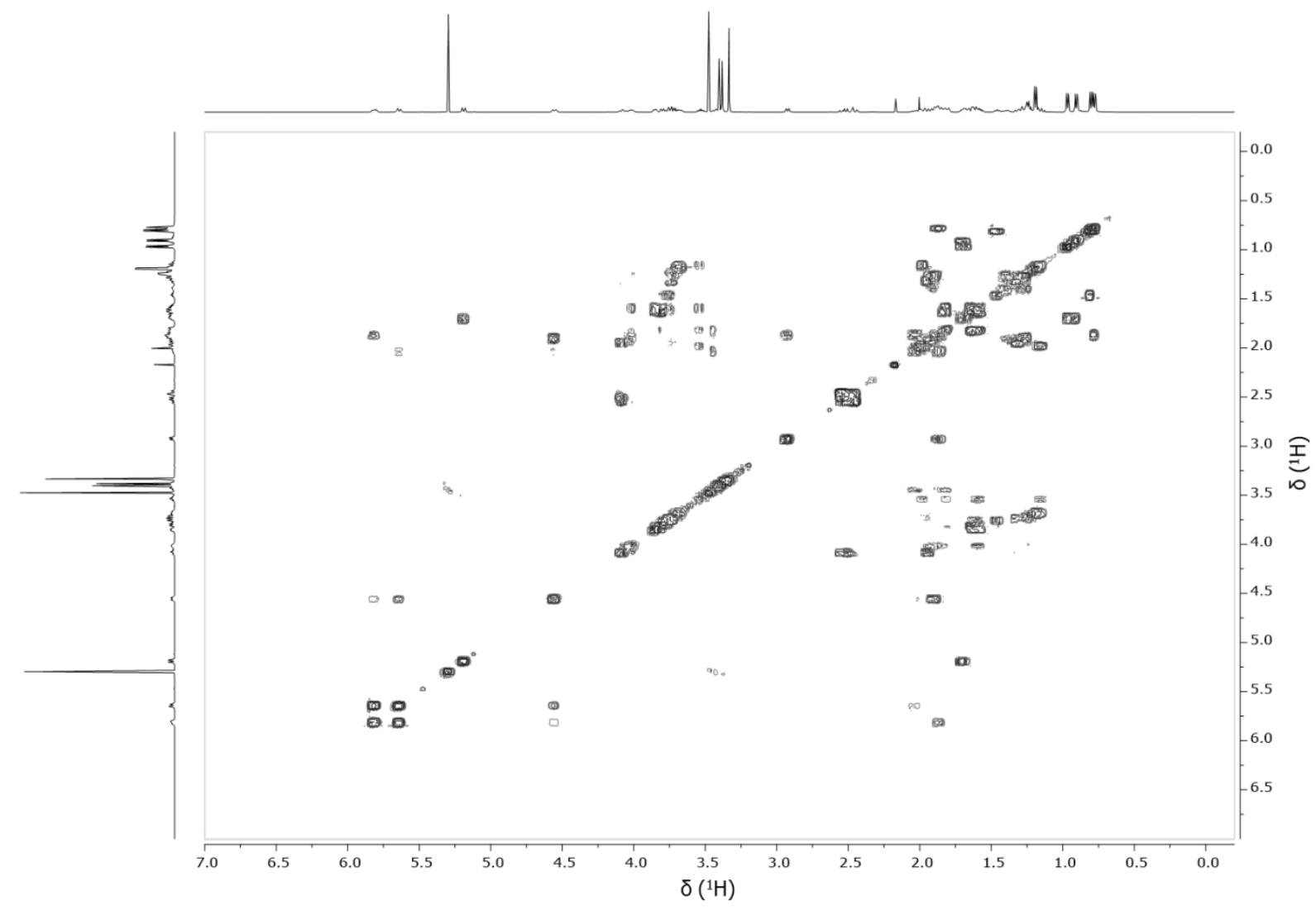

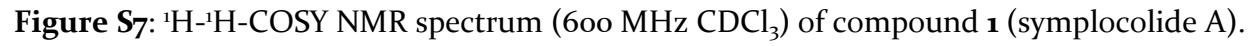

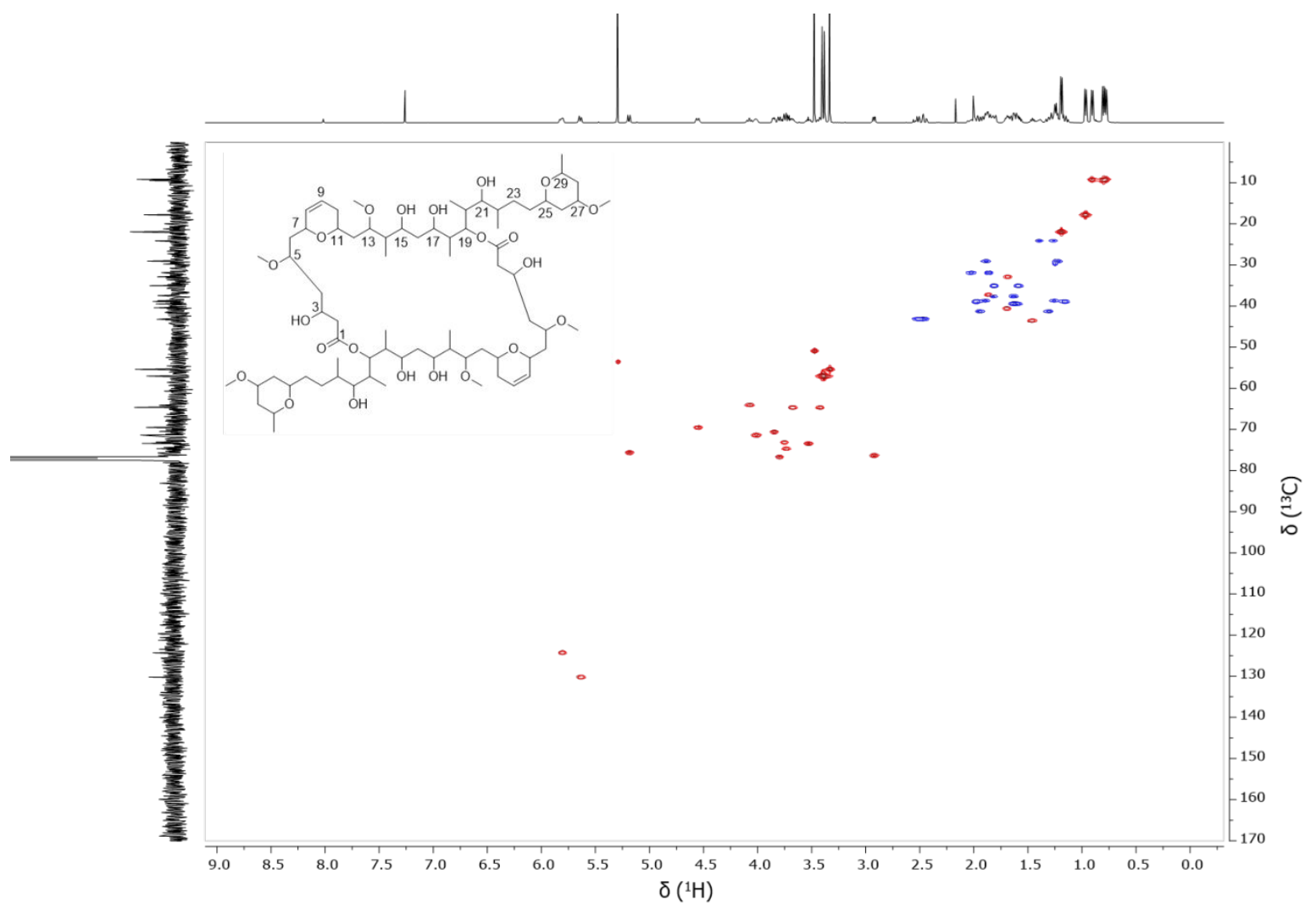

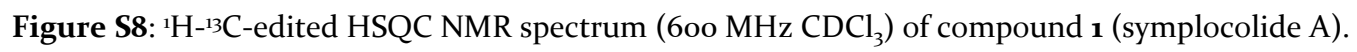




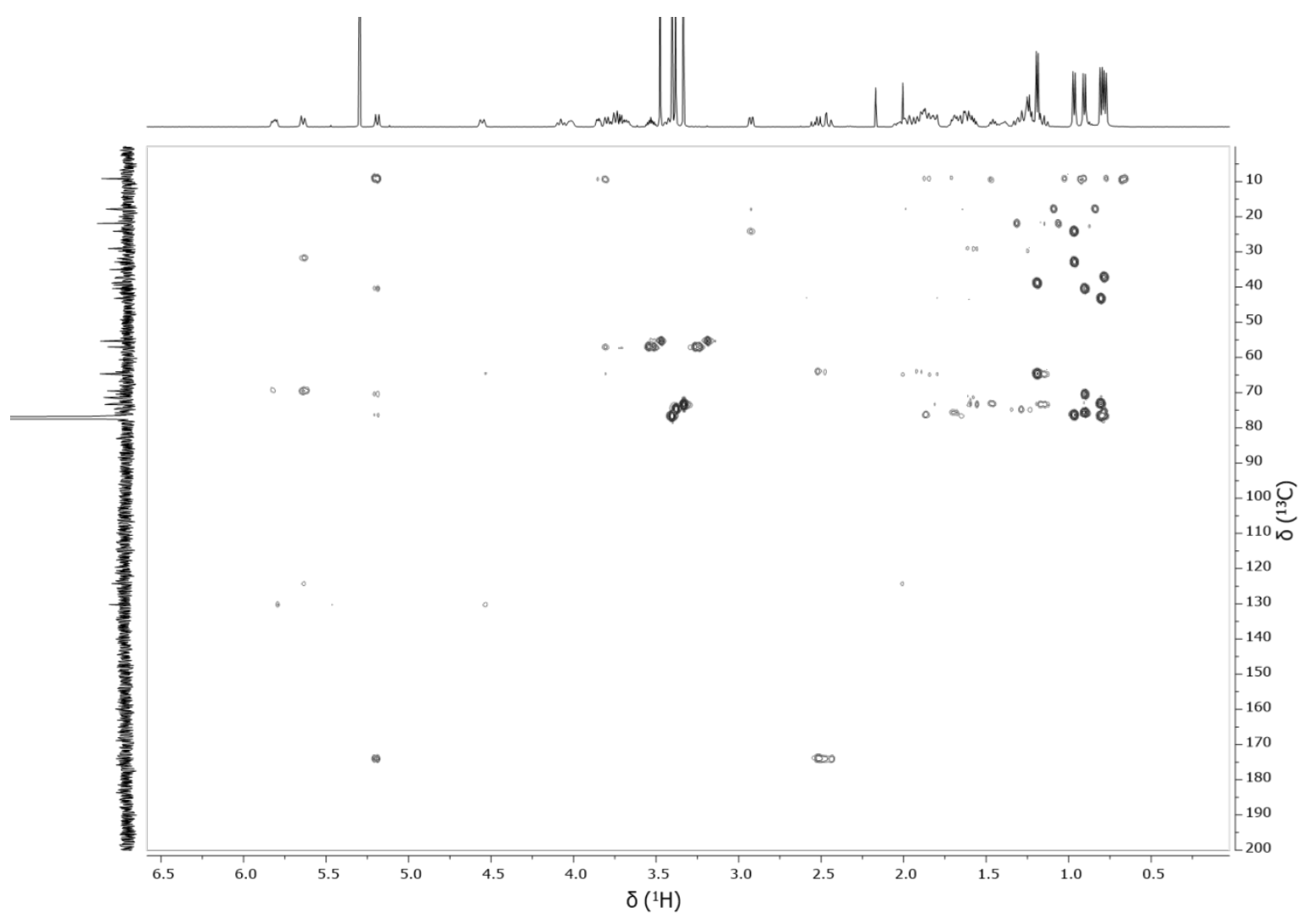

Figure S9: ${ }^{1} \mathrm{H}-{ }^{13} \mathrm{C}-\mathrm{HMBC}$ NMR spectrum (6oo $\left.\mathrm{MHz} \mathrm{CDCl}_{3}\right)$ of compound $\mathbf{1}$ (symplocolide A).

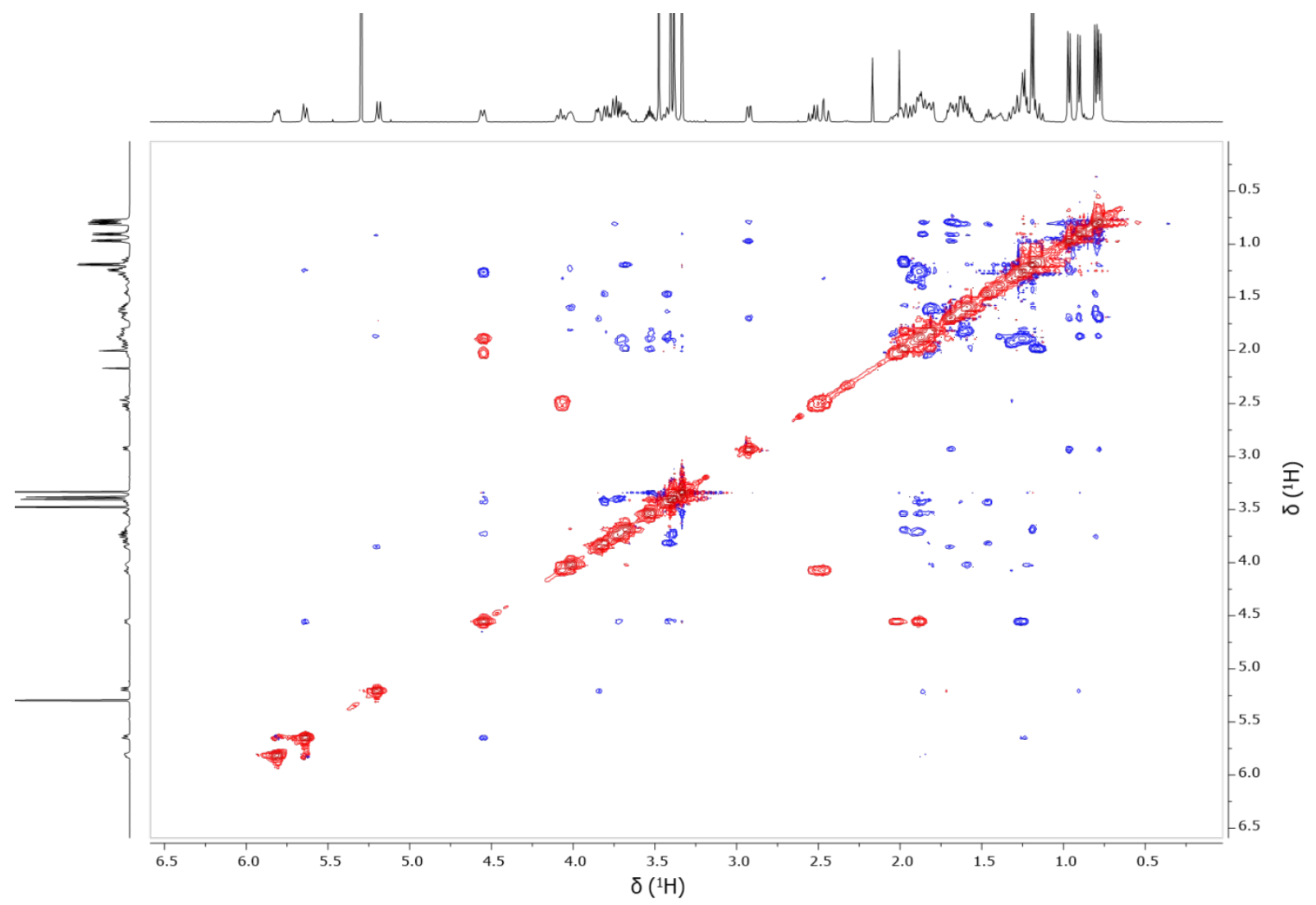

Figure S1o: ${ }^{1} \mathrm{H}-1 \mathrm{H}-\mathrm{ROESY}$ NMR spectrum (6oo $\mathrm{MHz} \mathrm{CDCl}_{3}$ ) of compound $\mathbf{1}$ (symplocolide A; red correlations are TOCSY peaks). 
Table S1: NMR assignment for compound 1 (symplocolide A) in $\mathrm{CDCl}_{3}$ (50o $\mathrm{MHz}$ )

\begin{tabular}{|c|c|c|c|c|c|}
\hline No $C / H^{[b]}$ & $\delta_{C^{[a]}}$, mult & $\delta_{\mathrm{H}}^{[\mathrm{a}]}($ mult,$J$ in $\mathrm{Hz})$ & ${ }^{1} \mathrm{H}-{ }^{1} \mathrm{H}-\mathrm{COSY}$ & ${ }^{1} \mathrm{H}-{ }^{13} \mathrm{C}-\mathrm{HMBC}$ & ${ }^{1} \mathrm{H}-{ }^{1} \mathrm{H}-\mathrm{ROESY}$ \\
\hline $1,1^{\prime}$ & 174.o, C & & & $2,19^{\prime}$ & \\
\hline $2,2^{\prime}$ & 43.1, $\mathrm{CH}_{2}$ & $2.52 \mathrm{~m}, 2.45 \mathrm{dd}(2.5,16.4)$ & 3 & 1,3 & $3^{[c]}, 4$ \\
\hline $3,3^{\prime}$ & $64.1, \mathrm{CH}$ & 4.08 t (10.6) & 2,4 & 2,4 & $2^{[c]}, 4$ \\
\hline $4,4^{\prime}$ & $41.25, \mathrm{CH}_{2}$ & $1.94 \mathrm{~m}, 1.31 \mathrm{~m}$ & 3,5 & & 2,3 \\
\hline $5,5^{\prime}$ & $74.68, \mathrm{CH}$ & $3.74 \mathrm{~m}$ & 4,6 & 4, 5' -OMe & 5-OMe, 7 \\
\hline 5,5 -OMe & 57.1, $\mathrm{CH}_{3}$ & $3 \cdot 38 \mathrm{~s}$ & & 5 & 5,4 \\
\hline $6,6^{\prime}$ & $39 \cdot 5, \mathrm{CH}_{2}$ & $1.62 \mathrm{~m}, 1.57 \mathrm{~m}$ & & 11 & 4 (weak) \\
\hline $7,7^{\prime}$ & $69.5, \mathrm{CH}$ & $4.55 \mathrm{~d}(10.9)$ & $8,9,10$ & $8,9,11$ & $5,8,10^{[c]}, 11$ \\
\hline $8,8^{\prime}$ & 130.2, $\mathrm{CH}$ & $5.64 \mathrm{dt}(10.1)$ & $7,9,10$ & $7,9,10$ & $7,9,10,5$ \\
\hline $9,9^{\prime}$ & $124.26, \mathrm{CH}$ & $5.81 \mathrm{~m}$ & $7,8,10$ & 7,8 & 8,10 \\
\hline $10,10^{\prime}$ & $31.9 \mathrm{CH}_{2}$ & $2.02 \mathrm{~m}, 1.87 \mathrm{~m}$ & $7,8,9,11$ & $8,9,11$ & $7^{[c]}, 9$ (weak) \\
\hline $11,11^{\prime}$ & $64 \cdot 7, \mathrm{CH}$ & $3.44 \mathrm{~m}$ & 10 & $7,10,12$ & $7,10,13,14$ \\
\hline $12,12^{\prime}$ & $37 \cdot 6, \mathrm{CH}_{2}$ & $1.82 \mathrm{~m}, 1.63 \mathrm{~m}$ & 11,13 & 11,13 & 11 \\
\hline $13,13^{\prime}$ & 76.66, $\mathrm{CH}$ & $3.80 \mathrm{dt}(9.5,2.1)$ & 12,14 & 11, 13-OMe, 14-Me & 13-OMe, 14 \\
\hline 13, 13' -OMe & 57.0, $\mathrm{CH}_{3}$ & $3.40 \mathrm{~s}$ & & 13 & 12,13 \\
\hline $14,14^{\prime}$ & $43 \cdot 5, \mathrm{CH}$ & $1.46 \mathrm{~m}$ & 14-Me, 15 & 14-Me, 15 & $11,13,14-\mathrm{Me}$ \\
\hline 14, $14^{\prime}-\mathrm{Me}$ & $9 \cdot 5, \mathrm{CH}_{3}$ & o.8o d (7.0) & 14 & 14 & 14,15 \\
\hline $15,15^{\prime}$ & $73.12, \mathrm{CH}$ & $3.75 \mathrm{~m}$ & 14,16 & 14,16 & 13-OMe, 14-Мe, 16 \\
\hline $16,16^{\prime}$ & $39.44, \mathrm{CH}_{2}$ & $1.62 \mathrm{~m}$ & 15,17 & $14,15,17$ & 17 (weak) \\
\hline $17,17^{\prime}$ & $70.6, \mathrm{CH}$ & 3.85 dd $(8.0,8.0)$ & 16 & 16 (weak), 18-Me & 16 (weak), 18, 19 \\
\hline $18,18^{\prime}$ & $40.5, \mathrm{CH}$ & $1.70 \mathrm{~m}$ & $17,18-\mathrm{Me}, 19$ & 18-Me, 19 & 17,19 \\
\hline 18, $18^{\prime}-\mathrm{Me}$ & $9 \cdot 3, \mathrm{CH}_{3}$ & $0.91 \mathrm{~d}(7.0)$ & 18 & $17,18,19$ & $18,19,20$ \\
\hline $19,19^{\prime}$ & $75.6, \mathrm{CH}$ & $5.19 \mathrm{~d}(10.2)$ & 18,20 & $1,17,18,18-\mathrm{Me}, 21$ & $17,18,18-\mathrm{Me}, 20$ \\
\hline $20,20^{\prime}$ & $37.2, \mathrm{CH}$ & $1.87 \mathrm{~m}$ & 20-Me, 21 & 19 (weak), 20-Me & 19, 20-Me \\
\hline $20,20^{\prime}-\mathrm{Me}$ & $9.2, \mathrm{CH}_{3}$ & $0.78 \mathrm{~d}(6.9)$ & 20 & $18,20,21$ & $20,21,22$ \\
\hline $21,21^{\prime}$ & $76.3, \mathrm{CH}$ & $2.92 \mathrm{dd}(9.4,2.4)$ & 20,22 & $19,20,22,22-\mathrm{Me}$ & 20-Me, 22, 22-Me \\
\hline $22,22^{\prime}$ & $32.9, \mathrm{CH}$ & $1.69 \mathrm{~m}$ & 22-Me & 21 (weak), 22-Me & 21, 22-Me \\
\hline 22, 22' -Me & $17.8, \mathrm{CH}_{3}$ & $0.97 \mathrm{~d}(6.8)$ & 22 & 21 & 21,22 \\
\hline $23,23^{\prime}$ & 24.1, $\mathrm{CH}_{2}$ & $1.39 \mathrm{~m}, 1.27 \mathrm{~m}$ & 21,24 & 21, 22-Me & 24 \\
\hline $24,24^{\prime}$ & 29.1, $\mathrm{CH}_{2}$ & $1.90 \mathrm{~m}, 1.23 \mathrm{~m}$ & 23,25 & & 23,25 \\
\hline $25,25^{\prime}$ & $71.4, \mathrm{CH}$ & $4.01 \mathrm{~m}$ & 24,26 & 27-Ome, 24, 26 & 24, 26, 29 (weak) \\
\hline 26,26 & $35 \cdot 4, \mathrm{CH}_{2}$ & $1.82 \mathrm{~m}, 1.59 \mathrm{~m}$ & 25,27 & 27 & 25 \\
\hline $27,27^{\prime}$ & $73.4, \mathrm{CH}$ & $3.53 \mathrm{~m}$ & 28,30 & & \\
\hline 27, $27^{\prime}-\mathrm{OMe}$ & $55 \cdot 3, \mathrm{CH}_{3}$ & $3.33 \mathrm{~s}$ & & 27 & 27 (weak) \\
\hline $28,28^{\prime}$ & $38.9, \mathrm{CH}_{2}$ & $1.98 \mathrm{~m}, 1.16 \mathrm{~m}$ & 29,31 & 29-Me & \\
\hline $29,29^{\prime}$ & $64.6, \mathrm{CH}$ & $3.68 \mathrm{~m}$ & 28, 29-Me & 28, 29-Me & 28, 29-Me \\
\hline 29, 29' -Me & 21.9, $\mathrm{CH}_{3}$ & $1.20 \mathrm{~d}(6.2)$ & 28,29 & 28,29 & 29 \\
\hline
\end{tabular}

[a] Assignments are based on extensive 1D and 2D NMR measurements (HMBC, HSQC, COSY). See also Figures Si-6. [b] Numbers according to Figure $S_{1}$. [c] TOCSY cross peak. 


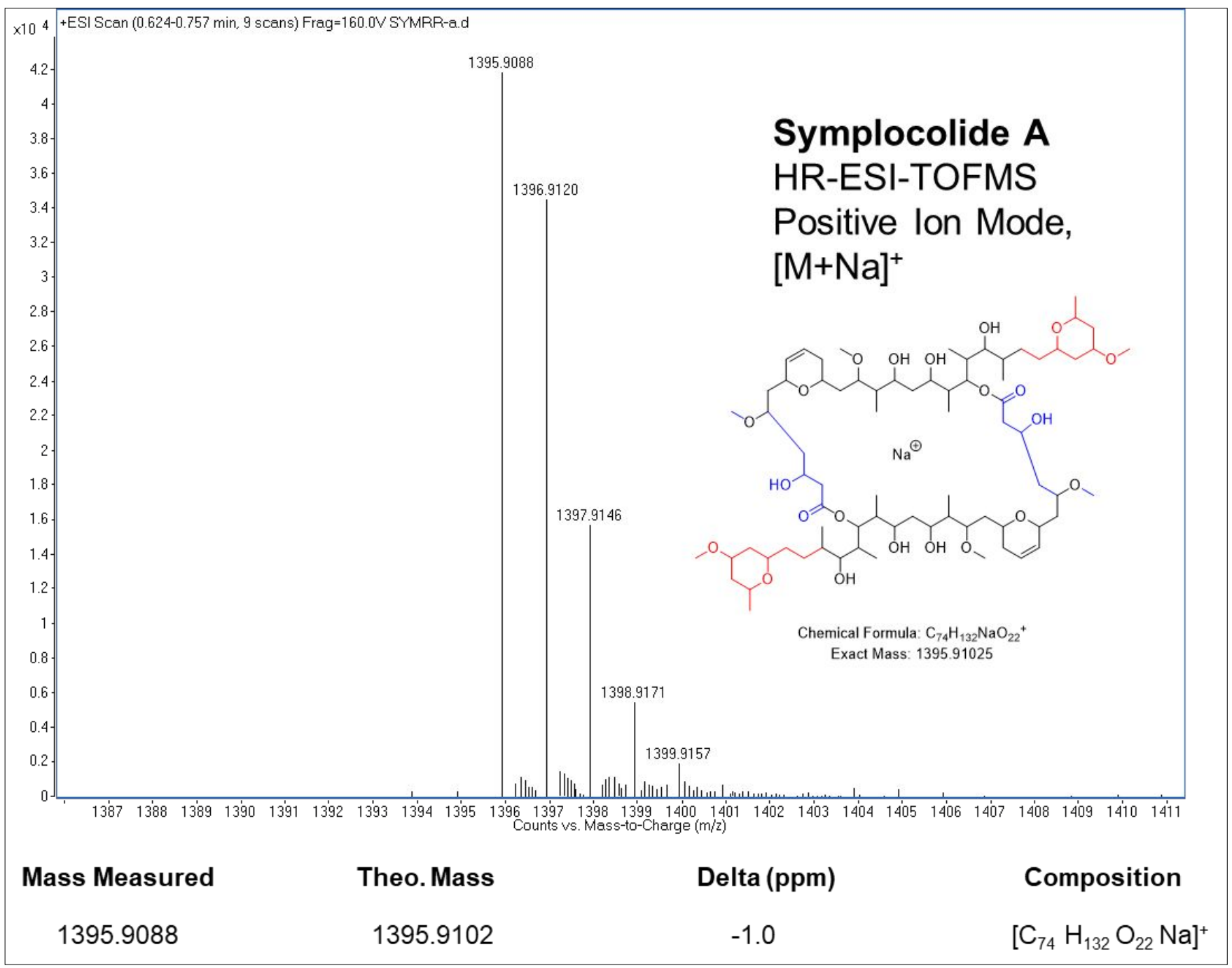

Figure S11. HR-ESI-ToF-MS measurements of compound 1 (symplocolide A).. For the sodium adduct we measured 1395.9088 (1395.9102 calculated) for yielding the sum formula $\mathrm{C}_{74} \mathrm{H}_{132} \mathrm{O}_{22} \mathrm{Na}^{+}(\Delta=-1.0 \mathrm{ppm})$.

Table S2. Table of ${ }^{13} \mathrm{C}$ NMR data comparing swinholide $\mathrm{A}^{7}$ (4), symplocolide A (1), and luminaolide $\mathrm{B}^{5}$ (3). Chemical shift differences $>$ 1ppm are highlighted in red.

\begin{tabular}{|c|c|c|c|c|c|c|}
\hline No. in 4 & $\begin{array}{l}\text { Compound } 4 \\
\left(\mathrm{CDCl}_{3}\right)\end{array}$ & $\begin{array}{l}\text { Compound } 1 \\
\left(\mathrm{CDCl}_{3}\right)\end{array}$ & $\begin{array}{c}\Delta \text { ppm } \\
\text { of } 1 \\
\text { and } 4\end{array}$ & No. in $1+3$ & $\begin{array}{l}\text { Compound } 3 \\
\left(\mathrm{CD}_{3} \mathrm{CN}\right)\end{array}$ & $\begin{array}{c}\Delta \text { ppm } \\
\text { of } 1 \text { and } 3\end{array}$ \\
\hline $1,1^{\prime}$ & 170.1 & 174.0 & 3.9 & $1,1^{\prime}$ & 174.7 & -0.7 \\
\hline $2,2^{\prime}$ & $113 \cdot 3$ & 43.1 & -- & $2,2^{\prime}$ & 43.6 & -0.5 \\
\hline $3,3^{\prime}$ & 153.3 & 64 & -- & $3,3^{\prime}$ & 65.4 & -1.4 \\
\hline $4,4^{\prime}$ & $134 \cdot 3$ & 41.25 & -- & $4,4^{\prime}$ & 43.2 & -1.95 \\
\hline $4,4^{\prime}-\mathrm{Me}$ & 12.3 & 74.68 & -- & $5,5^{\prime}$ & 75.2 & -0.51 \\
\hline $5,5^{\prime}$ & 142.3 & 57.1 & -- & 5, 5' -OMe & 56.9 & 0.2 \\
\hline $6,6^{\prime}$ & $37 \cdot 4$ & -- & -- & -- & -- & -- \\
\hline $7,7^{\prime}$ & 66.7 & -- & -- & -- & -- & -- \\
\hline
\end{tabular}




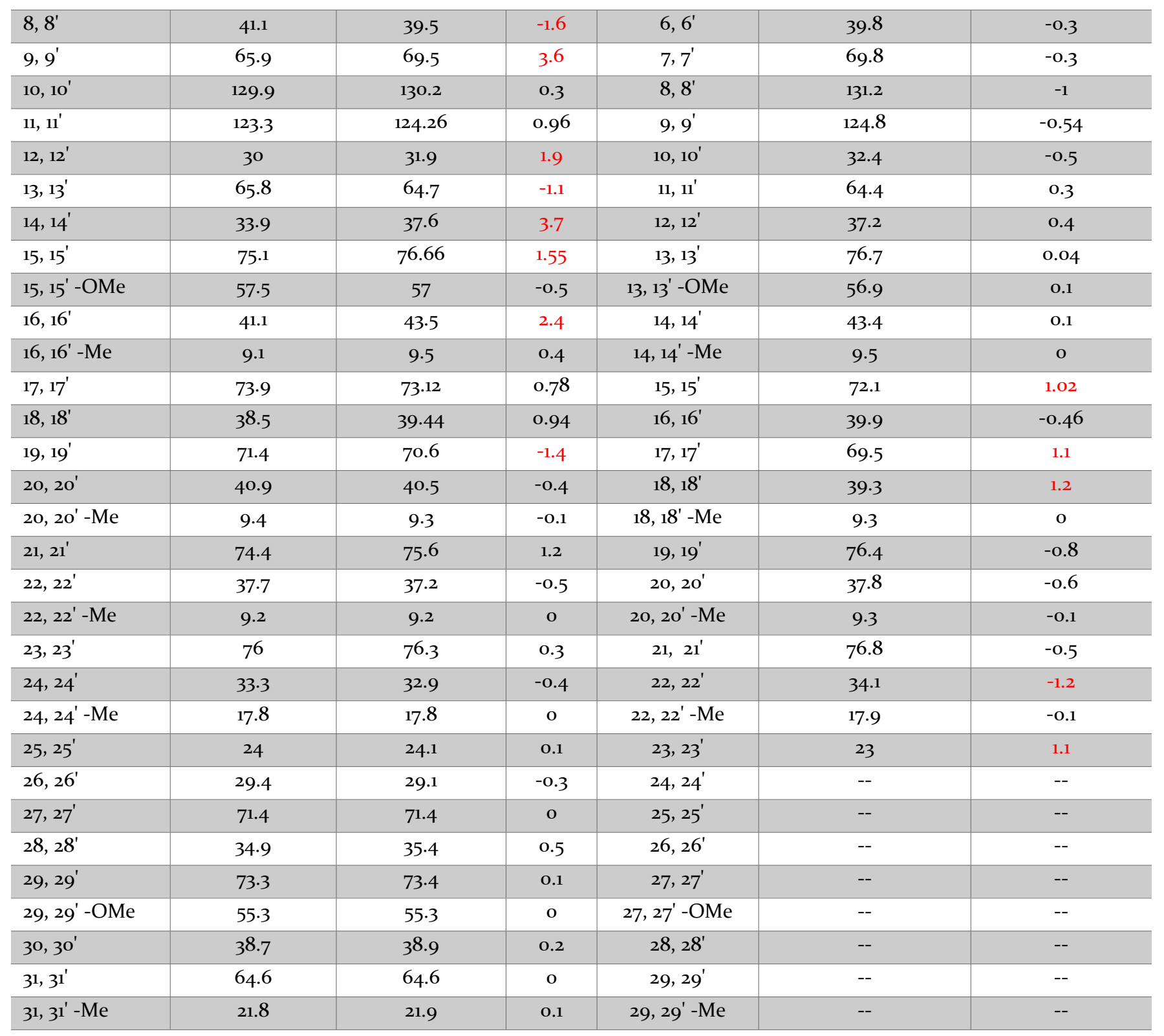


Table S3. Table of ${ }^{1} \mathrm{H}$ NMR data comparing swinholide $\mathrm{A}^{7}$ (4), symplocolide A (1), and luminaolide B5 (3).

\begin{tabular}{|c|c|c|c|c|}
\hline No. in 4 & Compound $4\left(\mathrm{CDCl}_{3}\right)$ & Compound $1\left(\mathrm{CDCl}_{3}\right)$ & No. in $1+3$ & Compound $_{3}\left(\mathrm{CD}_{3} \mathrm{CN}\right)$ \\
\hline $2,2^{\prime}$ & $5.79 \mathrm{~d}(15.8)$ & $2.53 \mathrm{~m}, 2.45 \mathrm{dd}(2.5,16.4)$ & $2,2^{\prime}$ & $2.4 \mathrm{~m}, 2.56 \mathrm{dd}(2.7,16.4)$ \\
\hline \multirow[t]{2}{*}{$3,3^{\prime}$} & $7.58 \mathrm{~d}(15.8)$ & $4.08 \mathrm{t}(10.6)$ & $3,3^{\prime}$ & $3.96 \mathrm{~m}$ \\
\hline & -- & $1.94 \mathrm{~m}, 1.31 \mathrm{~m}$ & $4,4^{\prime}$ & $1.77 \mathrm{~m}, 1.34 \mathrm{~m}$ \\
\hline 4, $4^{\prime}-\mathrm{Me}$ & $1.81 \mathrm{~s}$ & $3.74 \mathrm{~m}$ & $5,5^{\prime}$ & $3.58 \mathrm{~m}$ \\
\hline $5,5^{\prime}$ & $6.08 \mathrm{dd}(9.0,5.1)$ & $3.38 \mathrm{~s}$ & 5, 5'-OMe & $3.302 \mathrm{~s}$ \\
\hline $6,6^{\prime}$ & $\begin{array}{c}2.46 \text { ddd }(19.9,9.7,9.7) \\
2.17 \text { br d }(14.9)\end{array}$ & -- & -- & -- \\
\hline $7,7^{\prime}$ & $4.16 \mathrm{dd}(7.2,7.2)$ & -- & -- & -- \\
\hline $8,8^{\prime}$ & $1.60 \mathrm{~m}$ & $1.62 \mathrm{~m}, 1.57 \mathrm{~m}$ & $6,6^{\prime}$ & $1.85 \mathrm{~m}, 1.23 \mathrm{~m}$ \\
\hline $9,9^{\prime}$ & 4.52 br d (9.2) & $4.55 \mathrm{~d}(10.9)$ & $7,7^{\prime}$ & $4.39 \mathrm{~m}$ \\
\hline $10,10^{\prime}$ & $5.69 \mathrm{~d}(10.2)$ & $5.64 \mathrm{dt}(10.1)$ & $8,8^{\prime}$ & $5.66 \mathrm{~m}$ \\
\hline $11,11^{\prime}$ & $5.78 \mathrm{~m}$ & $5.81 \mathrm{~m}$ & $9,9^{\prime}$ & $5.80 \mathrm{~m}$ \\
\hline $12,12^{\prime}$ & $2.28 \mathrm{br} \mathrm{d}(17.2), 1.89 \mathrm{~m}$ & $2.02 \mathrm{~m}, 1.87 \mathrm{~m}$ & $10,10^{\prime}$ & $1.90 \mathrm{~m}$ \\
\hline $13,13^{\prime}$ & $3.90 \mathrm{~m}$ & $3.44 \mathrm{~m}$ & 11,11 & $3.49 \mathrm{~m}$ \\
\hline $14,14^{\prime}$ & $1.45 \mathrm{~m}, 2.15 \mathrm{~m}$ & $1.82 \mathrm{~m}, 1.63 \mathrm{~m}$ & $12,12^{\prime}$ & $1.73 \mathrm{~m}, 1.59 \mathrm{~m}$ \\
\hline $15,15^{\prime}$ & $4.01 \mathrm{~m}$ & $3.80 \mathrm{dt}(9.5,2.1))$ & $13,13^{\prime}$ & $3.89 \mathrm{~m}$ \\
\hline $15,15^{\prime}-\mathrm{OMe}$ & $3 \cdot 35, \mathrm{~s}$ & $3.40 \mathrm{~s}$ & 13, 13' -OMe & $3 \cdot 304 \mathrm{~s}$ \\
\hline $16,16^{\prime}$ & $1.68 \mathrm{~m}$ & $1.46 \mathrm{~m}$ & $14,14^{\prime}$ & $1.48 \mathrm{~m}$ \\
\hline $16,16 '-\mathrm{Me}$ & $0.81 \mathrm{~d}(6.9)$ & o.8o d (7.o) & $14,14^{\prime}-\mathrm{Me}$ & $0.77(d, 6.9)$ \\
\hline $17,17^{\prime}$ & 3.84 dd $(9.5,9.5)$ & $3.75 \mathrm{~m}$ & $15,15^{\prime}$ & $3.51 \mathrm{~m}$ \\
\hline $18,18^{\prime}$ & $1.63 \mathrm{~m}, 1.58 \mathrm{~m}$ & $1.62 \mathrm{~m}$ & $16,16^{\prime}$ & $1.77 \mathrm{~m}, 1.62 \mathrm{~m}$ \\
\hline $19,19^{\prime}$ & $4.01 \mathrm{~m}$ & 3.85 dd $(8.0,8.0)$ & $17,17^{\prime}$ & $3.80 \mathrm{~m}$ \\
\hline $20,20^{\prime}$ & $1.75 \mathrm{dq}(9.7,7.2)$ & $1.70 \mathrm{~m}$ & $18,18^{\prime}$ & $1.78 \mathrm{~m}$ \\
\hline 20, $20^{\prime}-\mathrm{Me}$ & $0.98 \mathrm{~d}(6.9)$ & $0.91 \mathrm{~d}(7.0)$ & 18,18 '-Me & $0.83(\mathrm{~d}, 6.9)$ \\
\hline $21,21^{\prime}$ & $5.35 \mathrm{~d}(10.8)$ & $5.19 \mathrm{~d}(10.2)$ & $19,19^{\prime}$ & $5.20(d, 10.0)$ \\
\hline $22,22^{\prime}$ & $1.93 \mathrm{~m}$ & $1.87 \mathrm{~m}$ & $20,20^{\prime}$ & $1.83 \mathrm{~m}$ \\
\hline $22,22^{\prime}-\mathrm{Me}$ & 0.83 d (6.9) & $0.78 \mathrm{~d}(6.9)$ & 20, 20' -Me & $0.81(\mathrm{~d}, 6.7)$ \\
\hline $23,23^{\prime}$ & $3.13 \mathrm{~d}(9.7)$ & $2.92 \mathrm{dd}(9.4,2.4)$ & $21,21^{\prime}$ & $2.98 \mathrm{~m}$ \\
\hline $24,24^{\prime}$ & $1.66 \mathrm{~m}$ & $1.69 \mathrm{~m}$ & $22,22^{\prime}$ & $1.62 \mathrm{~m}$ \\
\hline $24,24^{\prime}-\mathrm{Me}$ & $0.99 \mathrm{~d}(7.2)$ & $0.97 \mathrm{~d}(6.8)$ & 22, 22' -Me & $0.92(\mathrm{~d}, 6.9)$ \\
\hline $25,25^{\prime}$ & $1.38 \mathrm{~m}, 1.26 \mathrm{~m}$ & $1.39 \mathrm{~m}, 1.27 \mathrm{~m}$ & $23,23^{\prime}$ & $1.25 \mathrm{~m}$ \\
\hline 26,26 & $1.87 \mathrm{~m}, 1.25 \mathrm{~m}$ & $1.90 \mathrm{~m}, 1.23 \mathrm{~m}$ & $24,24^{\prime}$ & -- \\
\hline $27,27^{\prime}$ & $4.01 \mathrm{~m}$ & $4.01 \mathrm{~m}$ & $25,25^{\prime}$ & -- \\
\hline $28,28^{\prime}$ & $1.82 \mathrm{~m}, 1.59 \mathrm{~m}$ & $1.82 \mathrm{~m}, 1.59 \mathrm{~m}$ & $26,26^{\prime}$ & -- \\
\hline $29,29^{\prime}$ & $3.54 \mathrm{~m}$ & $3.53 \mathrm{~m}$ & $27,27^{\prime}$ & -- \\
\hline 29, 29' -OMe & $3 \cdot 33, \mathrm{~s}$ & $3.33 \mathrm{~s}$ & $27,27^{\prime}-\mathrm{OMe}$ & -- \\
\hline $30,30^{\prime}$ & $1.97 \mathrm{~m}, 1.17 \mathrm{~m}$ & $1.98 \mathrm{~m}, 1.16 \mathrm{~m}$ & $28,28^{\prime}$ & -- \\
\hline $31,31^{\prime}$ & $3.70 \mathrm{~m}$ & $3.68 \mathrm{~m}$ & $29,29^{\prime}$ & -- \\
\hline 31, 31' -Me & $1.20 \mathrm{~d}(5.9)$ & $1.20 \mathrm{~d}(6.2)$ & 29, 29' -Me & -- \\
\hline
\end{tabular}

aAndrianasolo et al7 bUeoka et al. 


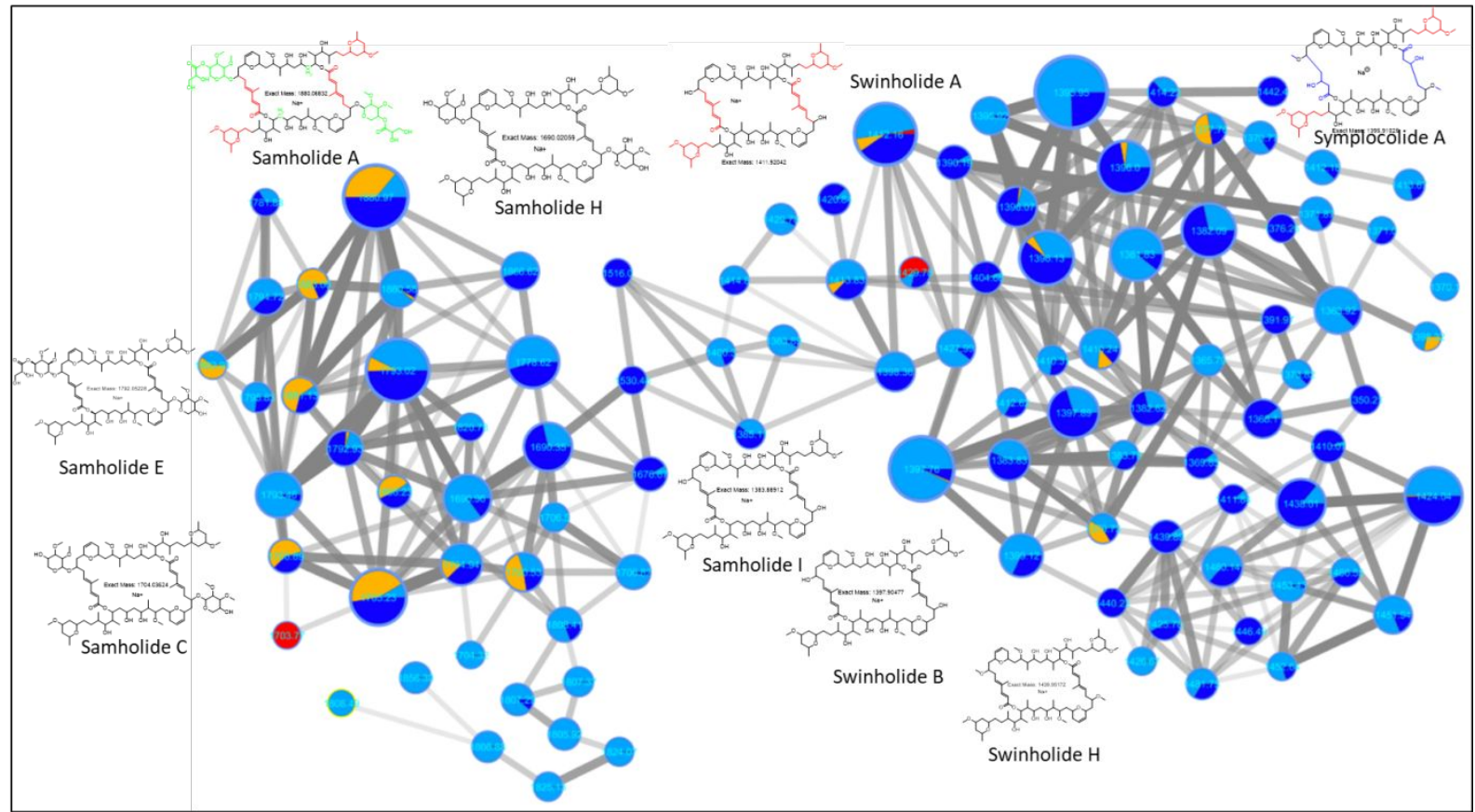

b)

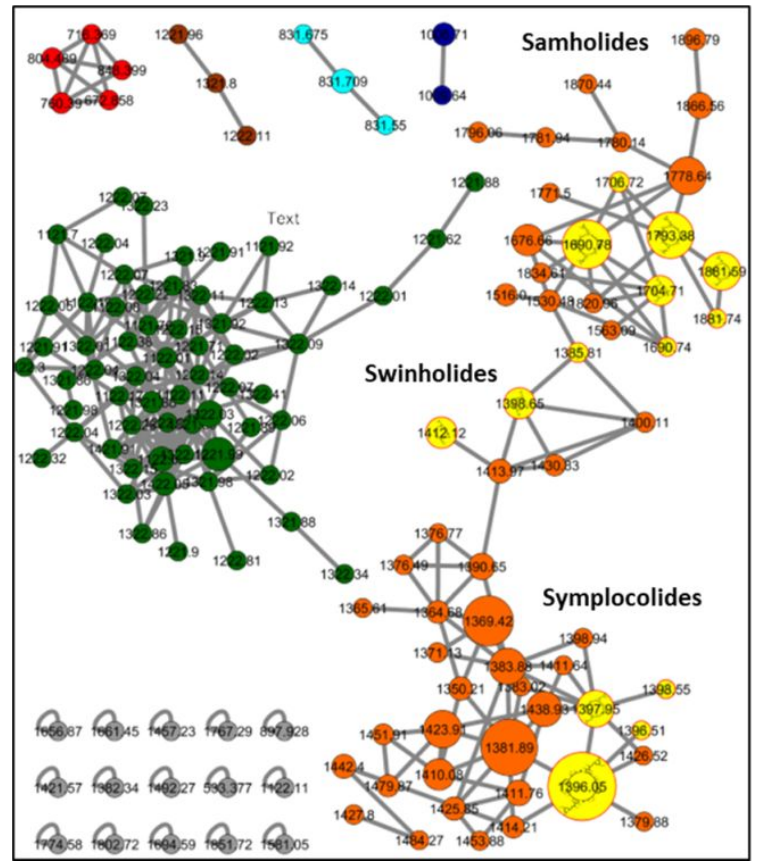

\begin{tabular}{|l|l|}
\hline Column & 규 CF_Dparent \\
Mapping Type & Discrete Mapping \\
Aminoglycosides & R:0 G:255 B:255 - \#00FFFF \\
Macrolides and analogues & R:255 G:102 B:0 - \#FF6600 \\
O-bromophenols & R:255 G:0 B:0 - \#FF0000 \\
Oligosaccharide sulfates & R:0 G:102 B:0 - \#006600 \\
Oligosaccharides & R:153 G:51 B:0 - \#993300 \\
Peptides & R:0 G:0 B:153 - \#000099 \\
no matches & R:153 G:153 B:153 - \#999... \\
\hline
\end{tabular}

Compound with GNPS Library ID

Figure S12: (a) MS²-based molecular networking of the cytotoxic fractions H + I reveals Swinholide A, B, H; Samholide A-I, Symplocolide A, as well as new analogs of all three classes. (b) MS²-based molecular networking enhanced with Networking Annotation Propagation and MolNetEnhancer (the colors represent different compound classes based on Classyfire, see in the box) of HPLC fractions of SPE fraction I2. GNPS library matches of swinholides, samholides, and symplocolides are labeled in the orange 'Macrolides and analogues' cluster. 

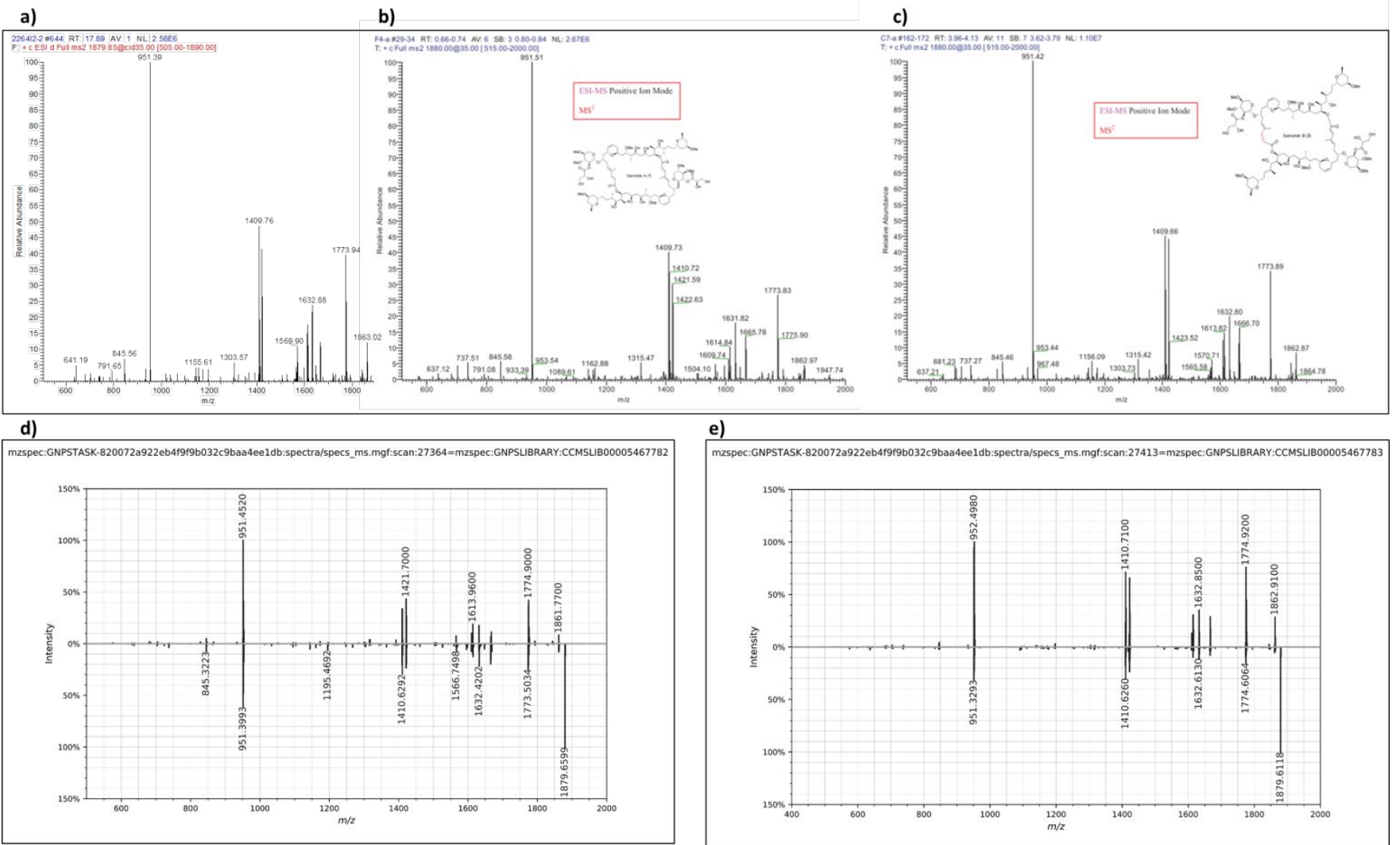

Figure S13. (a) ESI iontrap MS² spectrum of compounds 5 or 6 (samholide A or B) $[\mathrm{M}+\mathrm{Na}]^{+}$from this study and (b, c) reference ESI iontrap MS ${ }^{2}$ spectra $[\mathrm{M}+\mathrm{Na}]^{+}$of 5 (b) or $\mathbf{6}$ (c), respectively, from Tao et al. ${ }^{18} \mathrm{~d}$ ) Mirror plot of ESI iontrap MS 5 spectrum $[\mathrm{M}+\mathrm{Na}]^{+}$from this study and GNPS library spectrum $[\mathrm{M}+\mathrm{Na}]^{+}$. e) Mirror plot of ESI iontrap MS 6 spectrum $[\mathrm{M}+\mathrm{Na}]^{+}$from this study and GNPS library spectrum $[\mathrm{M}+\mathrm{Na}]^{+}$. 
mzspec:GNPSTASK-e41860bffa6f4a7fa3c347a96a698702:spectra/specs_ms.mgf:scan:202=mzspec:GNPSLIBRARY:CCMSLIB00005463857

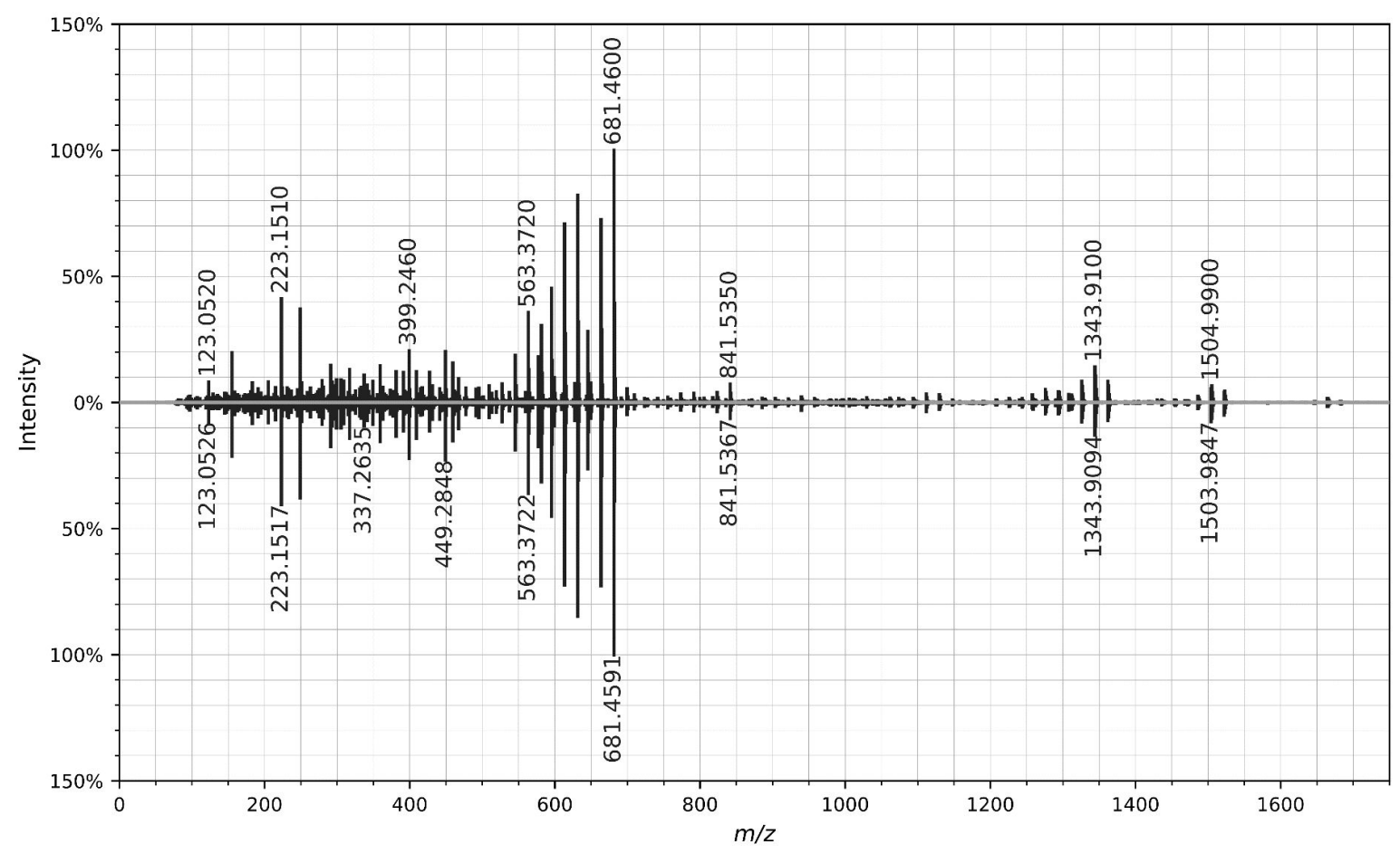

Figure S14: Mirror plot of high-resolution q-ToF MS² spectra $[\mathrm{M}+\mathrm{H}]^{+}$of compound $\mathbf{7}$ or 8 (samholide C or D) from this study and GNPS library spectrum $[\mathrm{M}+\mathrm{H}]^{+}$for $\mathbf{7} / \mathbf{8}$. 


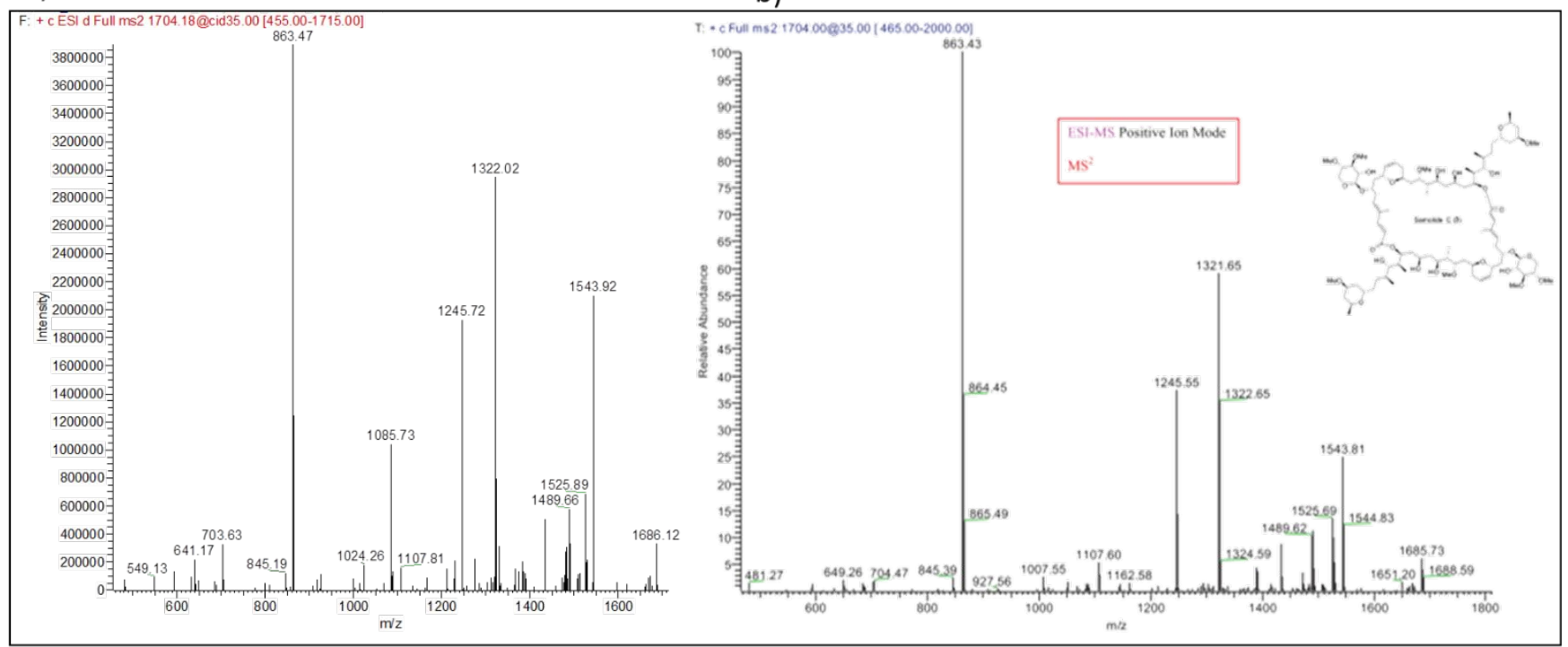

c)

mzspec:GNPSTASK-820072a922eb4f9f9b032c9baa4ee1db:spectra/specs_ms.mgf:scan:22822=mzspec:GNPSLIBRARY:CCMSLIB00005467784

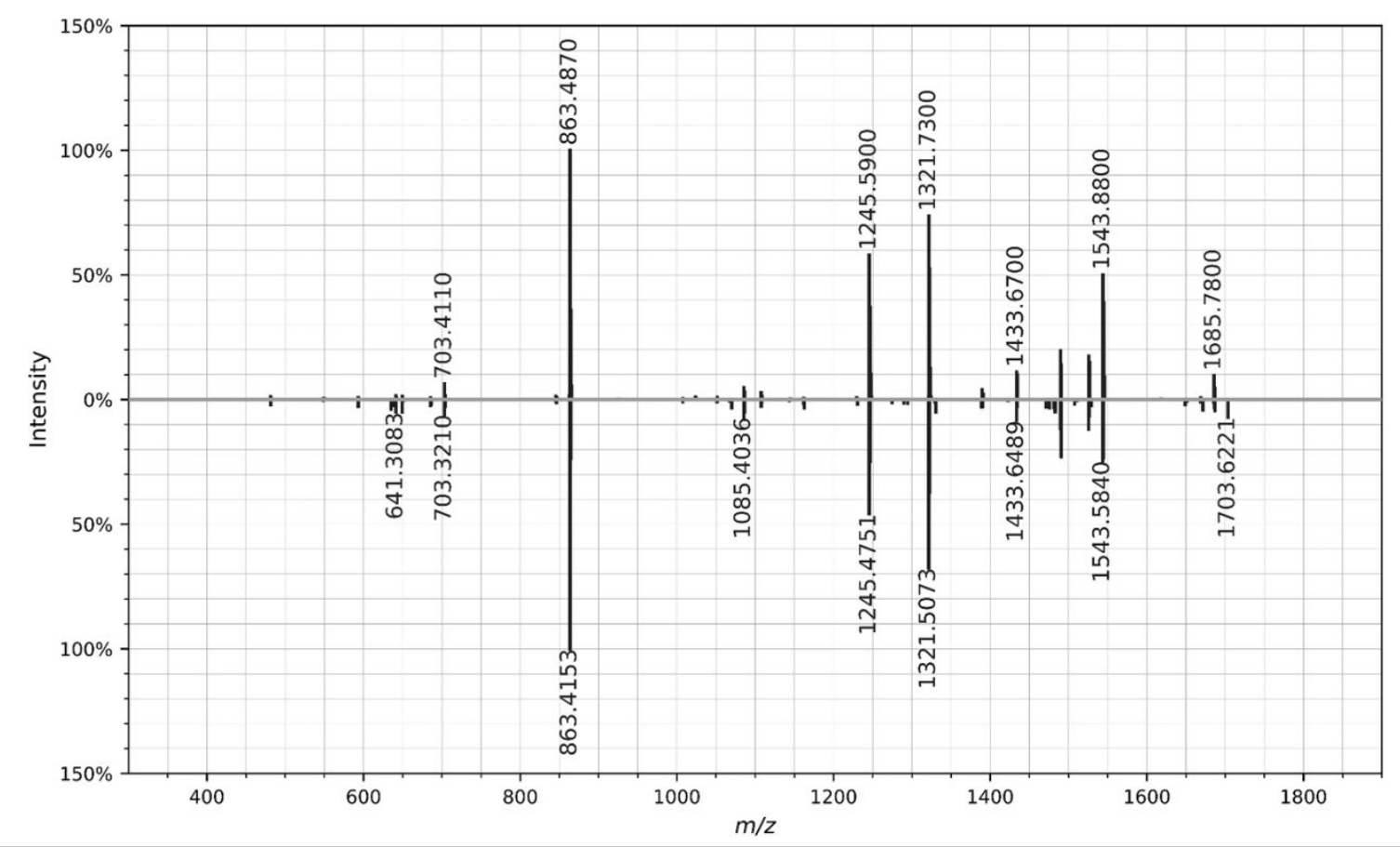

Figure S15. (a) ESI iontrap MS² spectrum of compound 7 (samholide C) $[\mathrm{M}+\mathrm{Na}]^{+}$from this study and (b) reference ESI iontrap $\mathrm{MS}^{2}$ spectrum $[\mathrm{M}+\mathrm{Na}]^{+}$from Tao et al. ${ }^{18} \mathrm{c}$ ) Mirror plot of ESI iontrap MS ${ }^{2}$ spectrum of 7 [M+Na] from this study and GNPS library spectrum $[\mathrm{M}+\mathrm{Na}]^{+}$of 7 . 


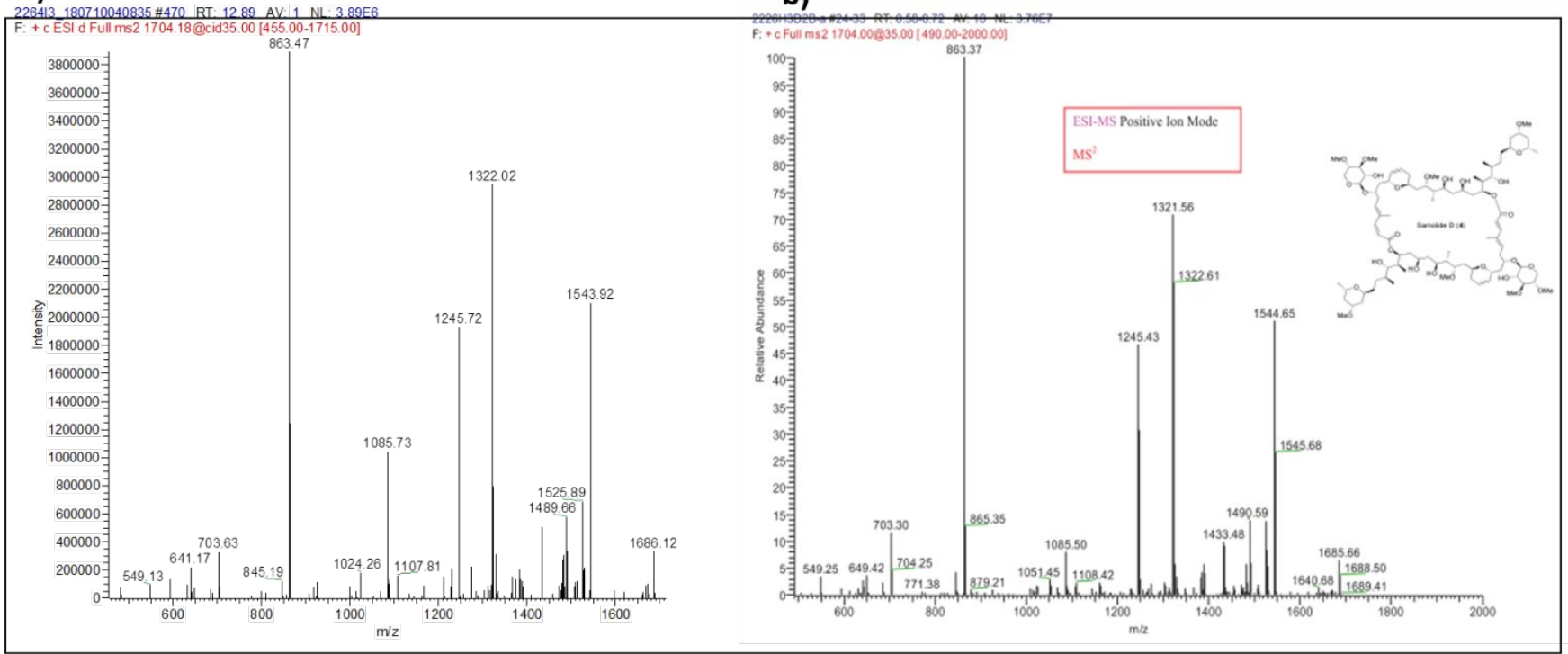

c)

mzspec:GNPSTASK-c4ef17ab0f1a40a1a97bbef42a898549:spectra/specs_ms.mgf:scan:8676=mzspec:GNPSLIBRARY:CCMSLIB00005467786

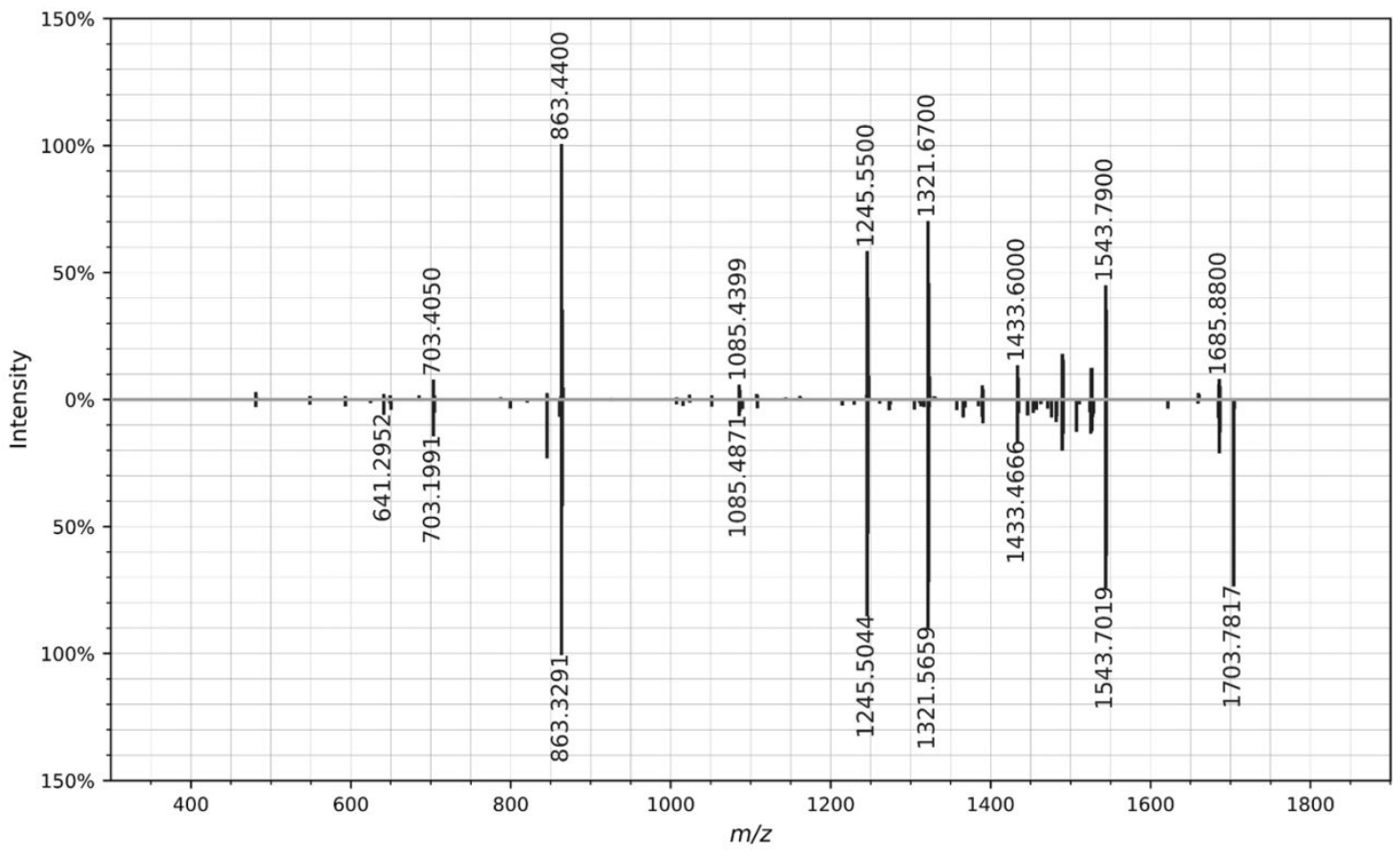

Figure S16. (a) ESI iontrap MS² spectrum of compound 8 (samholide D) $[\mathrm{M}+\mathrm{Na}]^{+}$from this study and (b) reference ESI iontrap $\mathrm{MS}^{2}$ spectrum $[\mathrm{M}+\mathrm{Na}]^{+}$from Tao et al. ${ }^{18} \mathrm{c}$ ) Mirror plot of ESI iontrap $\mathrm{MS}^{2}$ spectrum of $\mathbf{8}[\mathrm{M}+\mathrm{Na}]^{+}$from this study and GNPS library spectrum $[\mathrm{M}+\mathrm{Na}]^{+}$of 8. 


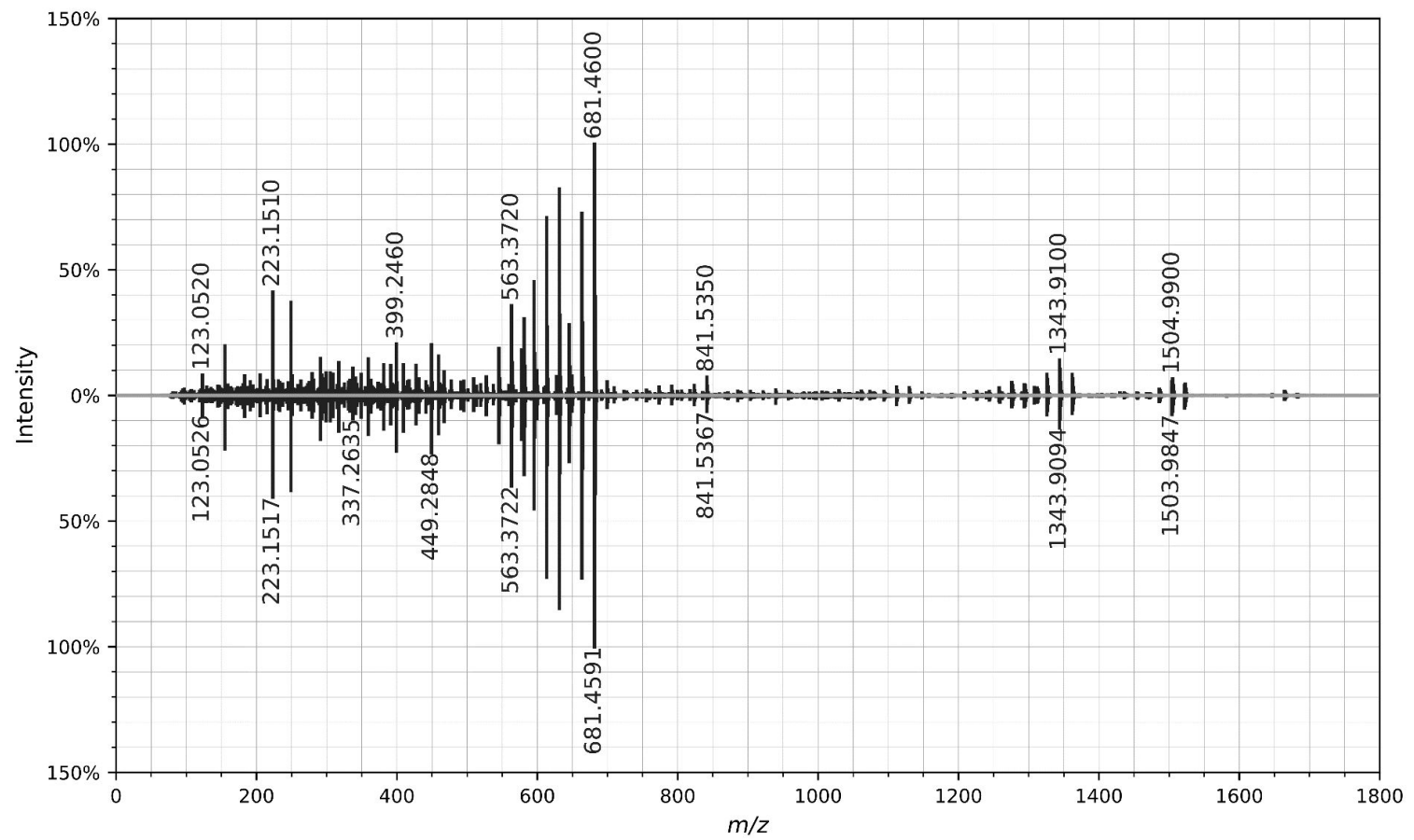

Figure S17. Mirror plot of high-resolution q-ToF MS² spectra $[\mathrm{M}+\mathrm{H}]^{+}$of samholide E, F or G from this study and GNPS library spectrum $[\mathrm{M}+\mathrm{H}]^{+}$of samholide $\mathrm{E} / \mathrm{F} / \mathrm{G}$. 
a)

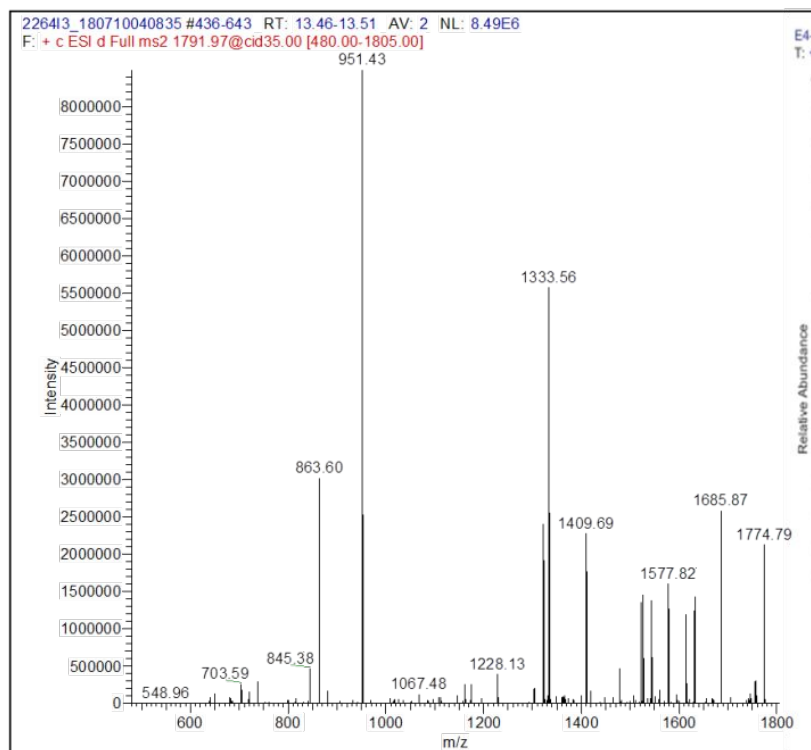

b)

E4-a \#89-97 RT:227-2.40 AV: 9 SB: 62.13 .223 NL: 3.31E6

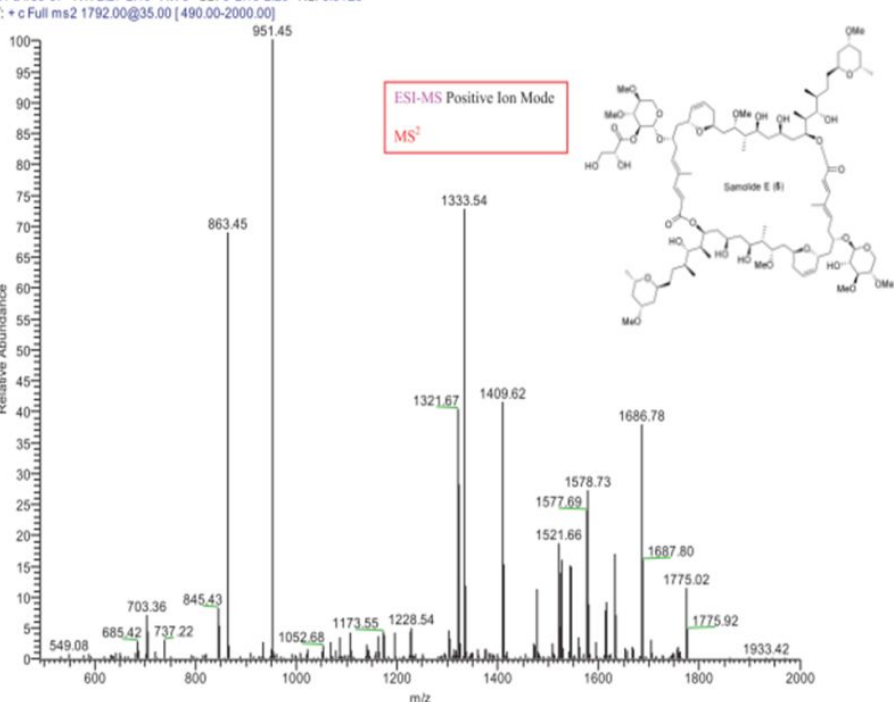

c)

mzspec:GNPSTASK-5b0a1d56a2ef4be3810259277a1ee772:spectra/specs_ms.mgf:scan:8199=mzSpec:GNPSLIBRARY:CCMSLIB00005467788

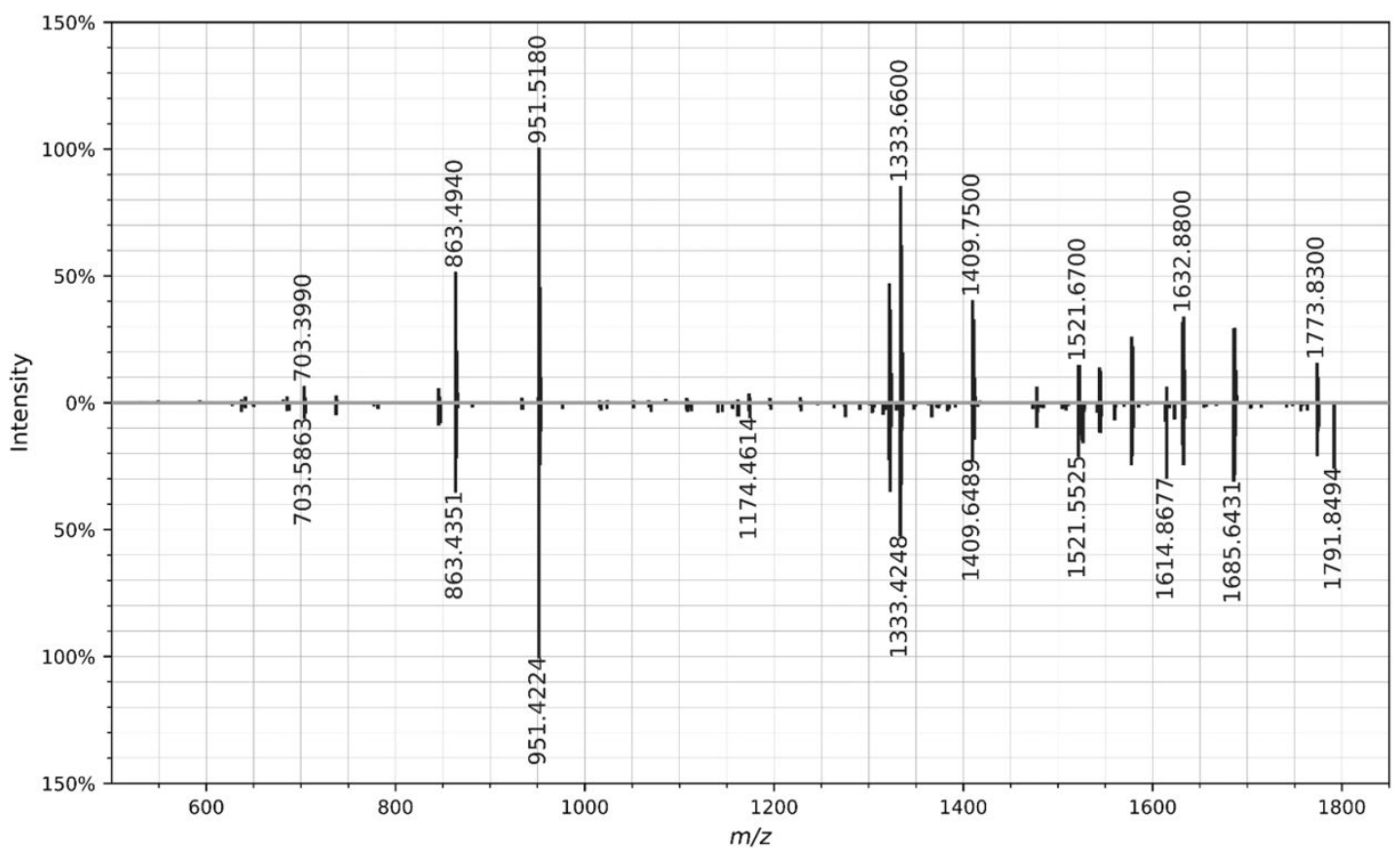

Figure S18.: (a) ESI iontrap $\mathrm{MS}^{2}$ spectrum of samholide $\mathrm{E}[\mathrm{M}+\mathrm{Na}]^{+}$from this study and (b) reference ESI iontrap $\mathrm{MS}^{2}$ spectrum $[\mathrm{M}+\mathrm{Na}]^{+}$from Tao et al. ${ }^{18} \mathrm{c}$ ) Mirror plot of ESI iontrap $\mathrm{MS}^{2}$ samholide E spectrum $[\mathrm{M}+\mathrm{Na}]^{+}$from this study and GNPS library spectrum $[\mathrm{M}+\mathrm{Na}]^{+}$for samholide $\mathrm{E}$ 
a)

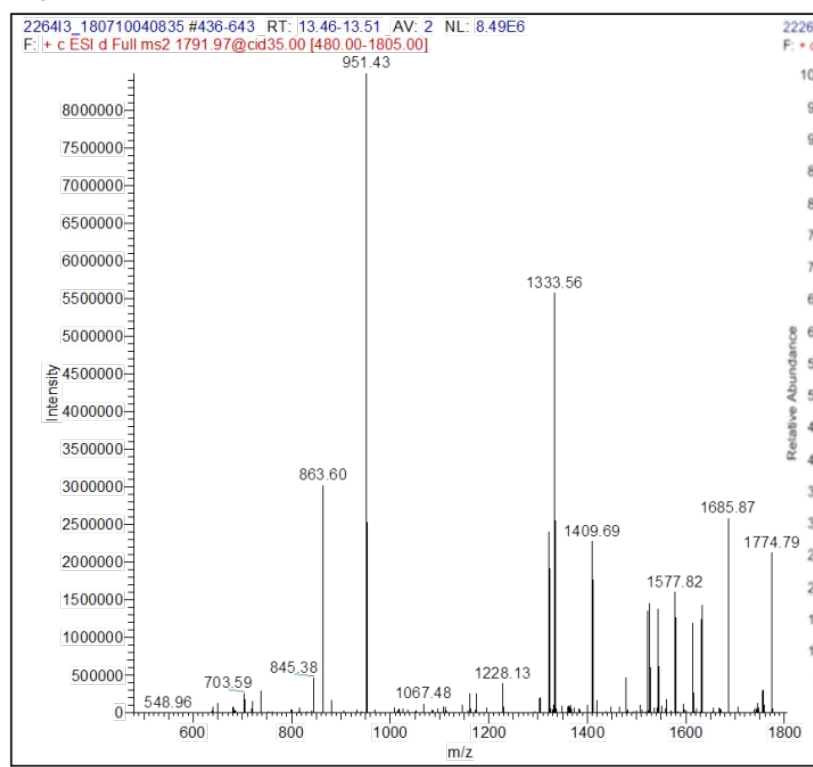

b)

126H3C7A1-a $125-28$ RT: 0.61-0.66 AV. 4 NL: 1.93E6

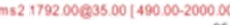

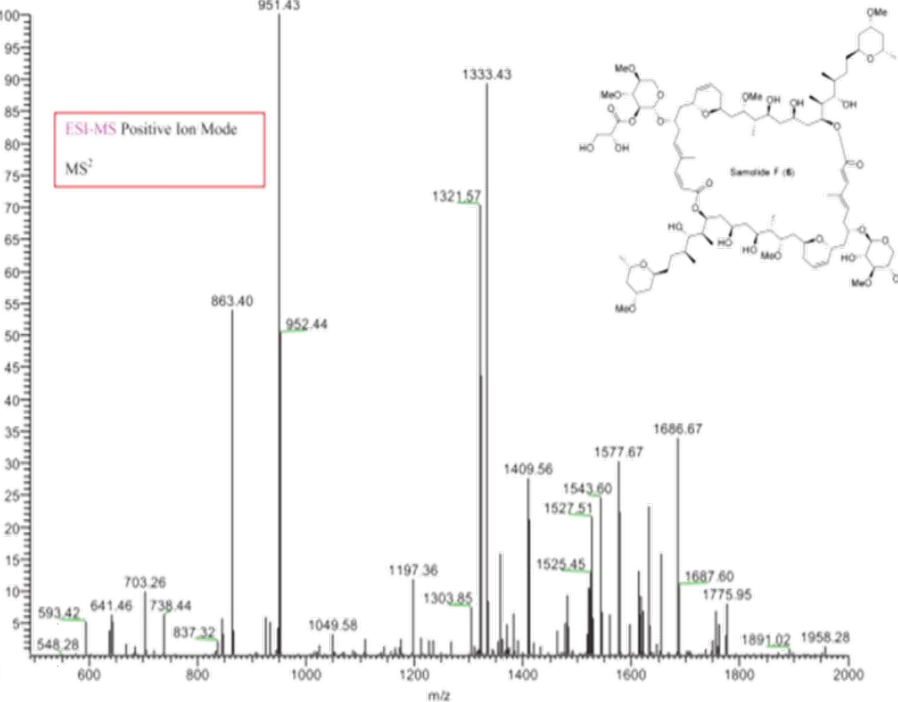

Figure S19. (a) Putative ESI iontrap $\mathrm{MS}^{2}$ spectrum of samholide F $[\mathrm{M}+\mathrm{Na}]^{+}$from this study and (b) reference ESI iontrap MS ${ }^{2}$ spectrum $[\mathrm{M}+\mathrm{Na}]^{+}$from Tao et al. ${ }^{18}$ 
a)

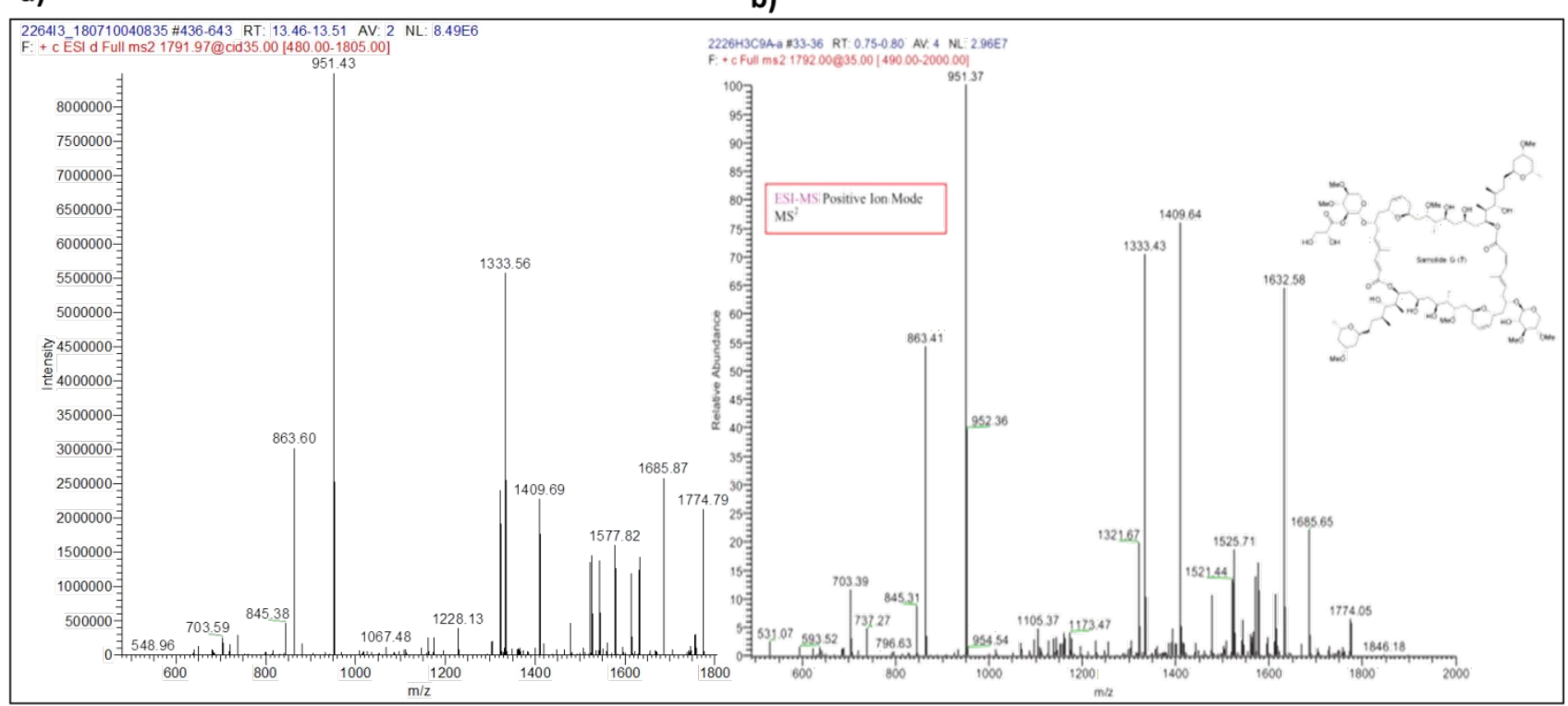

c)

mzspec:GNPSTASK-23a4eb9146c544c0bc5c0b1f323cb088:spectra/specs_ms.mgf:scan:9286=mzspec:GNPSLIBRARY:CCMSLIB00005467792

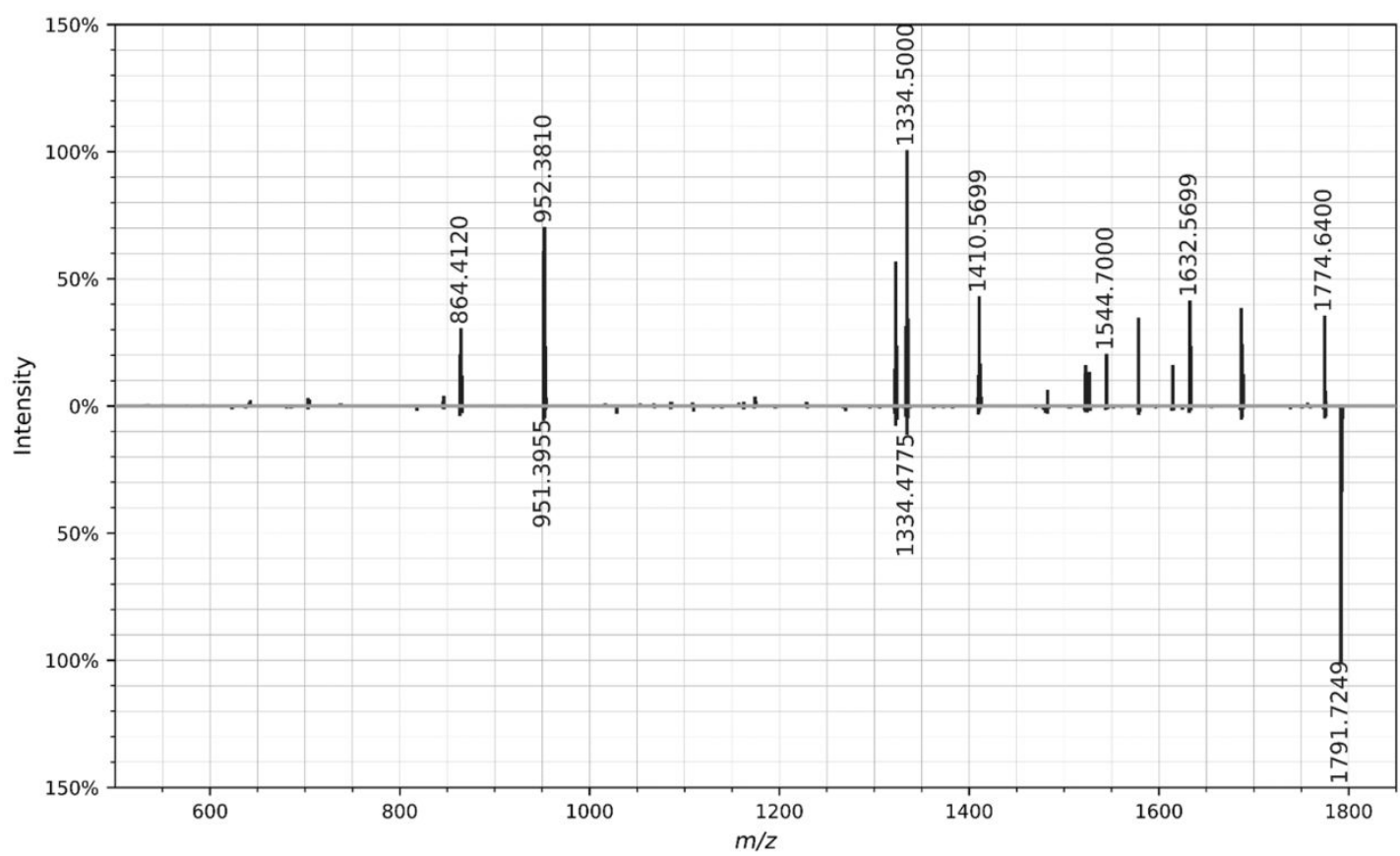

Figure S2o.: (a) ESI iontrap $\mathrm{MS}^{2}$ spectrum of samholide $\mathrm{G}[\mathrm{M}+\mathrm{Na}]^{+}$from this study and (b) reference ESI iontrap MS ${ }^{2}$ spectrum $[\mathrm{M}+\mathrm{Na}]^{+}$from Tao et $\mathrm{al}^{18} \mathrm{c}$ ) Mirror plot of ESI iontrap MS ${ }^{2}$ samholide G spectrum $[\mathrm{M}+\mathrm{Na}]^{+}$from this study and GNPS library spectrum $[\mathrm{M}+\mathrm{Na}]^{+}$of samholide $\mathrm{G}$. 
a)

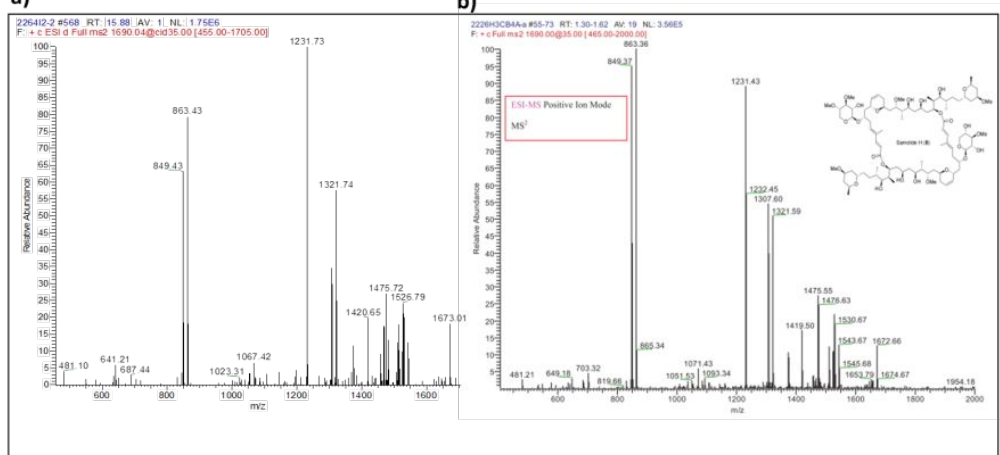

c)

mzspec:GNPSTASK-23a4eb9146c544c0bc5c0b1f323cb088:spectra/specs_ms.mgf:scan:7554=mzspec:GNPSLIBRARY:CCMSLIB00005436029

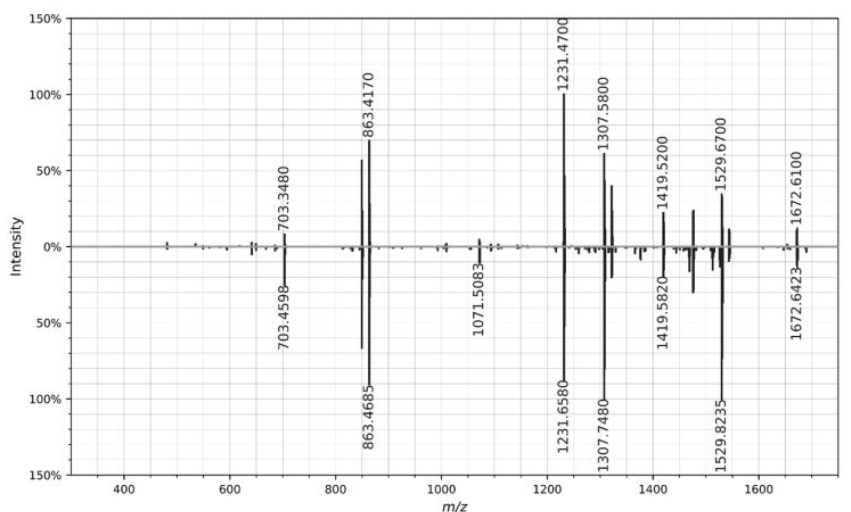

d)

mzspec:GNPSTASK-a512e66158494137a8c1cee91aced2bd:spectra/specs_ms.mgf:scan:119=mzspec:GNPSLIBRARY:CCMSLIB00005463859

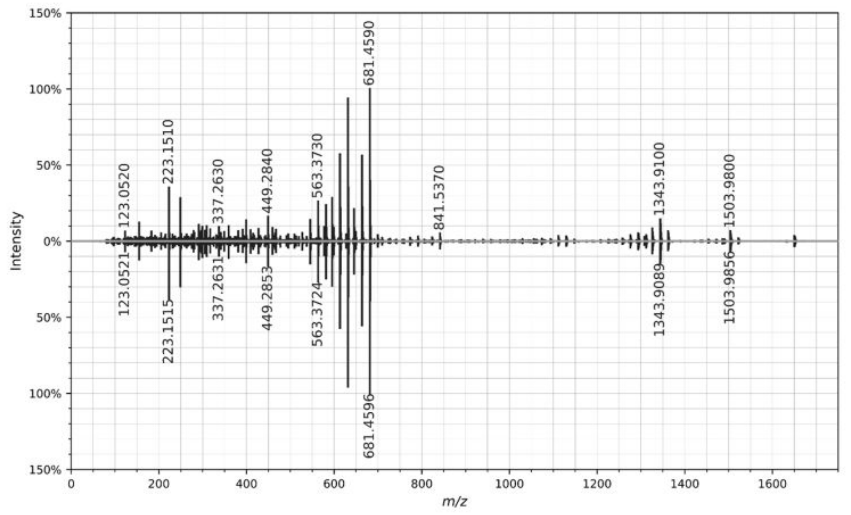

Figure S21. (a) ESI iontrap MS2 spectrum $[\mathrm{M}+\mathrm{Na}]^{+}$of samholide $\mathrm{H}$ from this study and (b) reference ESI iontrap $\mathrm{MS}^{2}$ spectrum $[\mathrm{M}+\mathrm{Na}]^{+}$from Tao et al. ${ }^{18} \mathrm{c}$ ) Mirror plot of ESI iontrap $\mathrm{MS}^{2}$ samholide $\mathrm{H}$ spectrum $[\mathrm{M}+\mathrm{Na}]^{+}$from this study and GNPS library spectrum $[\mathrm{M}+\mathrm{Na}]^{+}$. d) Mirror plot of high-resolution ESI q-ToF MS ${ }^{2}$ samholide $\mathrm{H}$ spectrum $[\mathrm{M}+\mathrm{Na}]^{+}$from this study and GNPS library spectrum $[\mathrm{M}+\mathrm{Na}]^{+}$samholide $\mathrm{H}$. 
a)

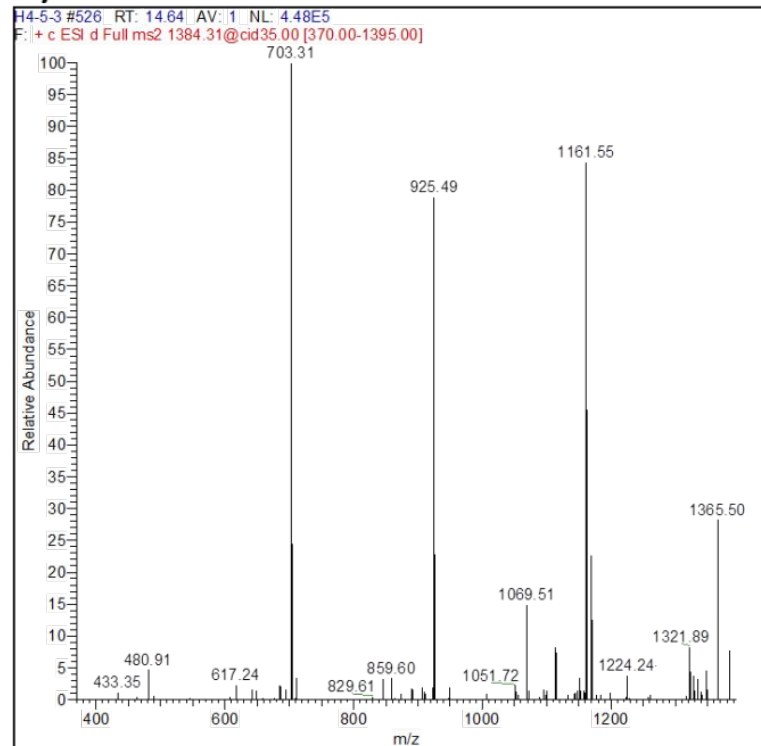

b)

2226H3C3-a $\# 31-34$ RT: 0.73-0.77 AV: 4 NL: $8.51 E 6$

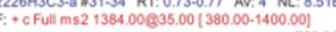

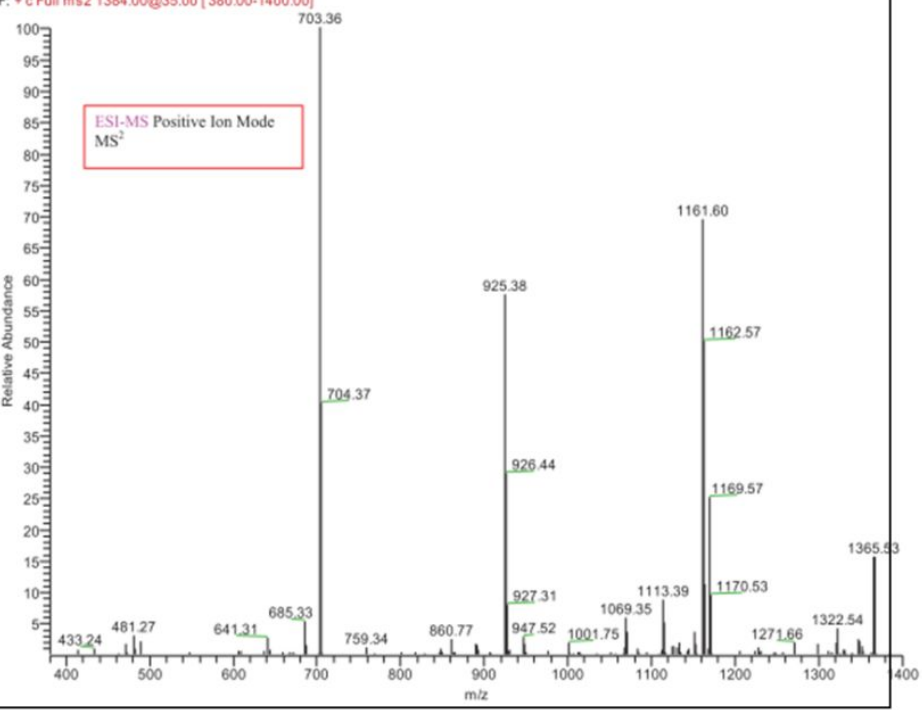

c)

mzspec:GNPSTASK-5b0a1d56a2ef4be3810259277a1ee772:spectra/specs_ms.mgf:scan:7256=mzspec:GNPSLIBRARY:CCMSLIB00005436030

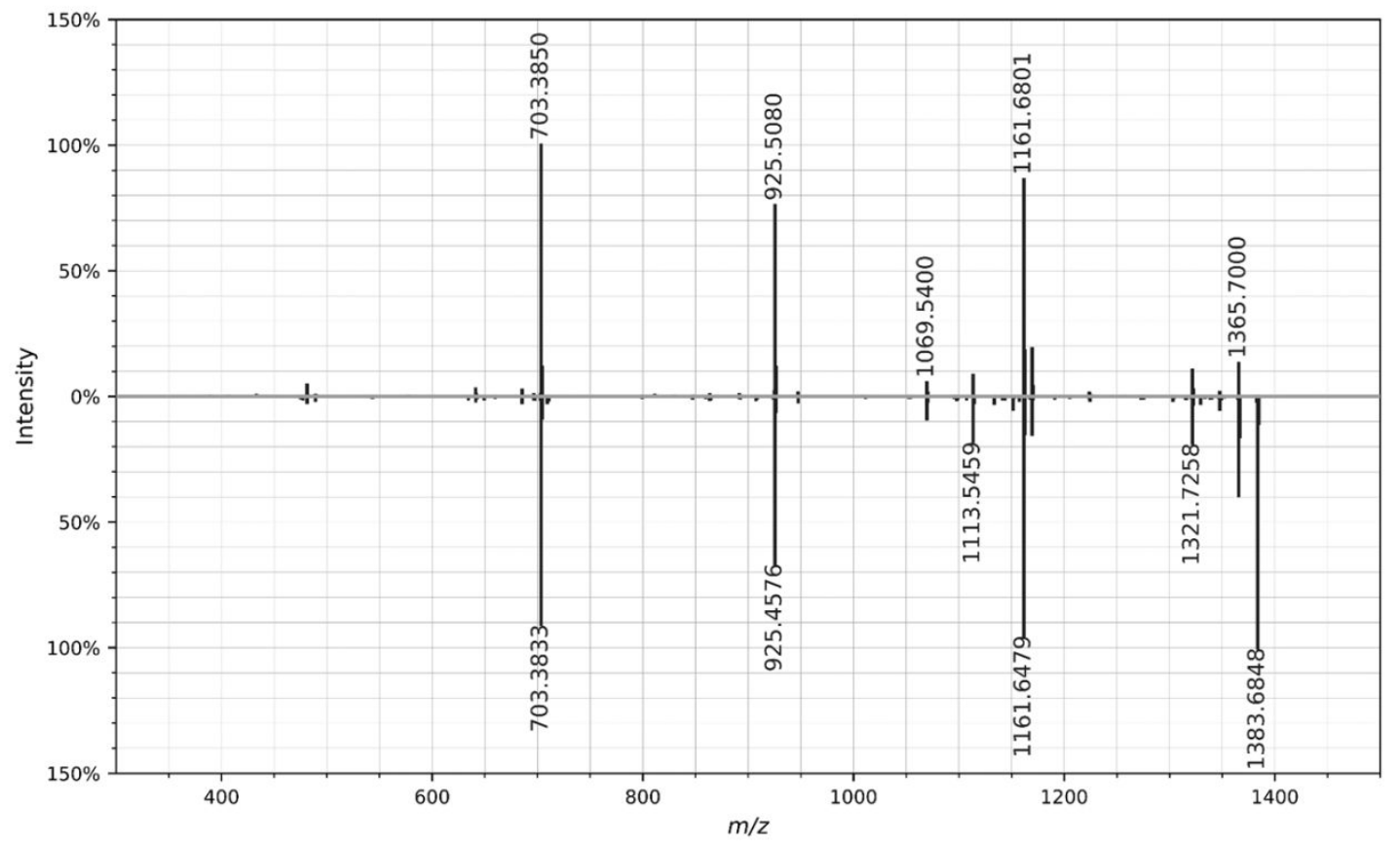

Figure S22. (a) ESI iontrap MS2 spectrum $[\mathrm{M}+\mathrm{Na}]^{+}$of samholide I from this study and (b) reference ESI iontrap MS2 spectrum $[\mathrm{M}+\mathrm{Na}]^{+}$from Tao et al..$^{18} \mathrm{c}$ ) Mirror plot of ESI iontrap $\mathrm{MS}^{2}$ samholide $\mathrm{H}$ spectrum $[\mathrm{M}+\mathrm{Na}]^{+}$from this study and GNPS library spectrum $[\mathrm{M}+\mathrm{Na}]^{+}$of samholide I. 
a)

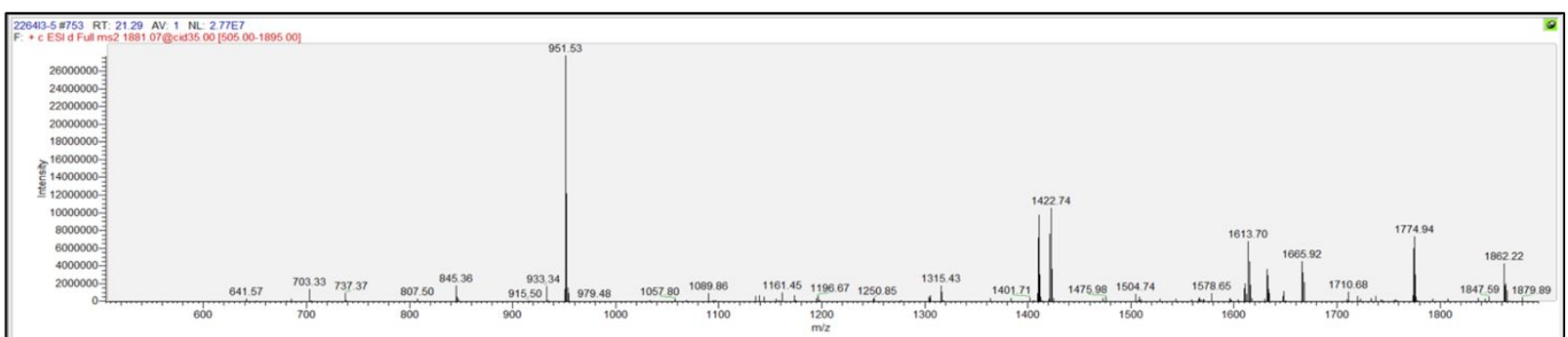

b)

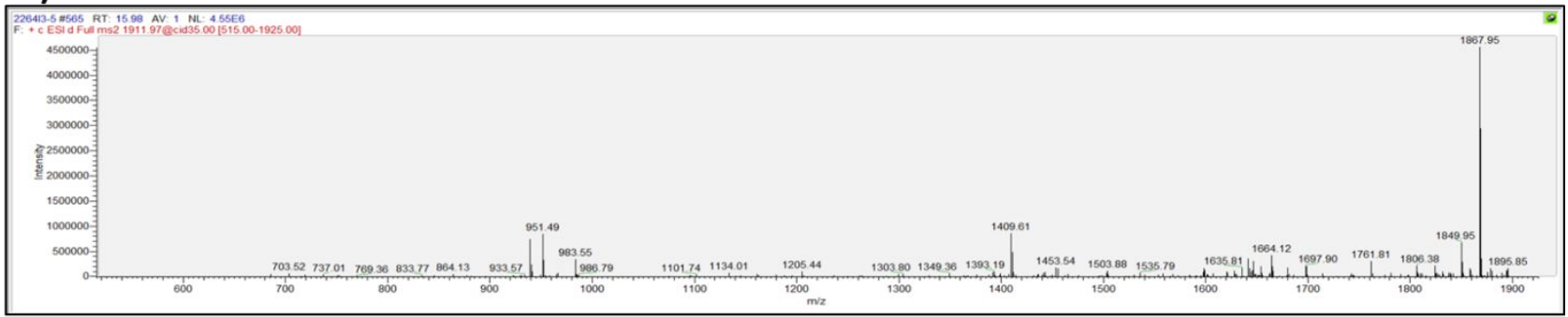

c)

mzspec:GNPSTASK-c4ef17ab0f1a40ala97bbef42a898549:spectra/specs_ms.mgf:scan:12181

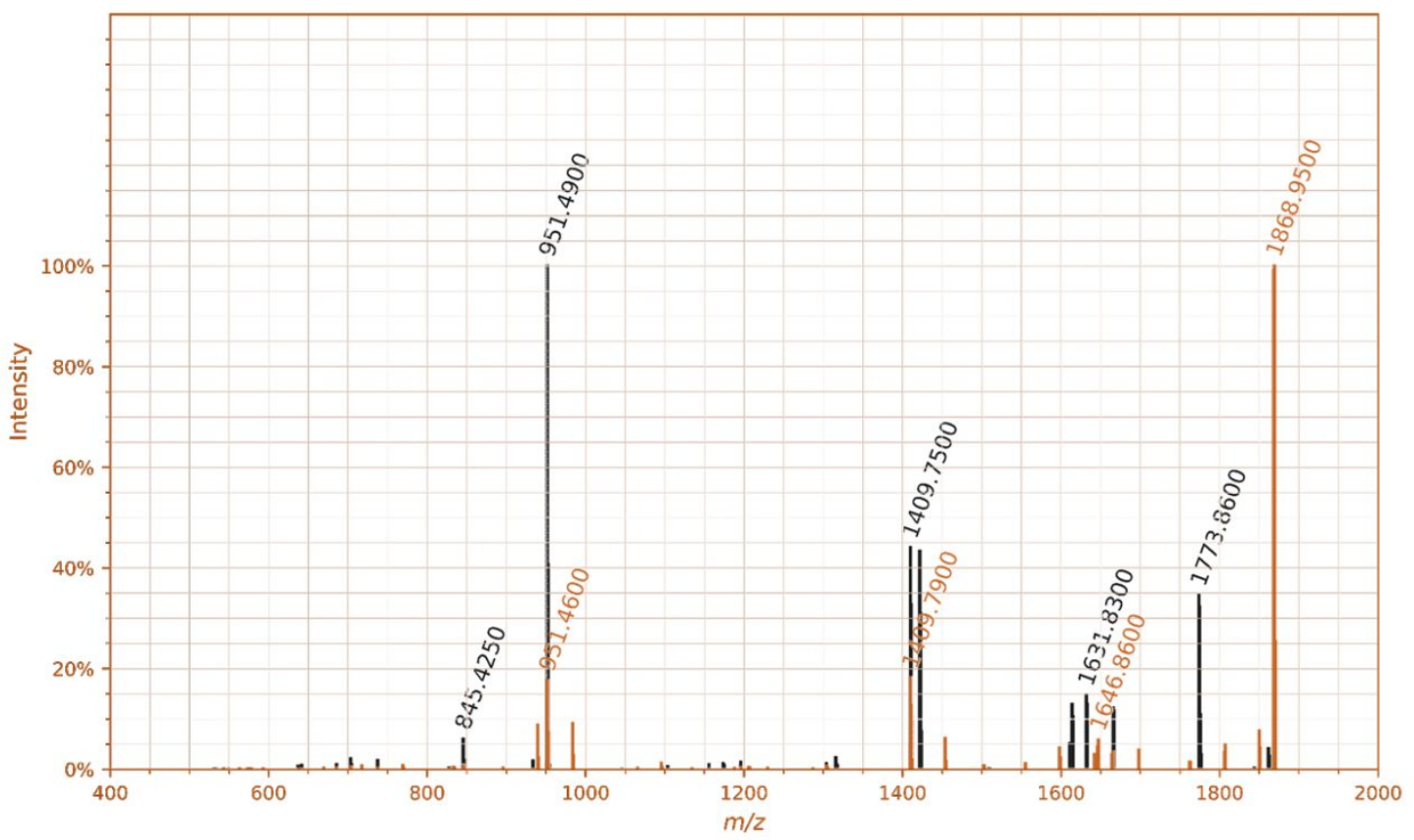

Figure S23: (a) ESI iontrap MS² spectra $[\mathrm{M}+\mathrm{Na}]^{+}$of samholide A/B (black) and (b) putatively new Samholide J (marron, $m / z=1911.9$ ). c) Overlay plot of a) + b) (same study different $\mathrm{MS}^{2}$ scans). 
a)

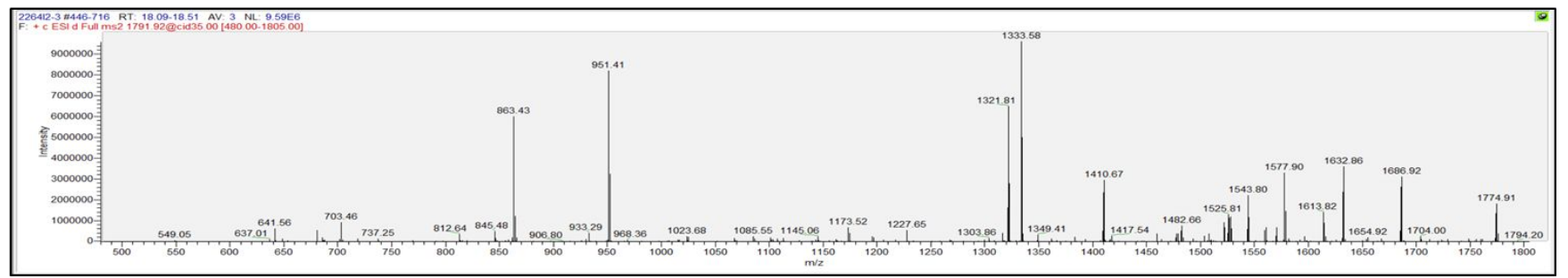

b)

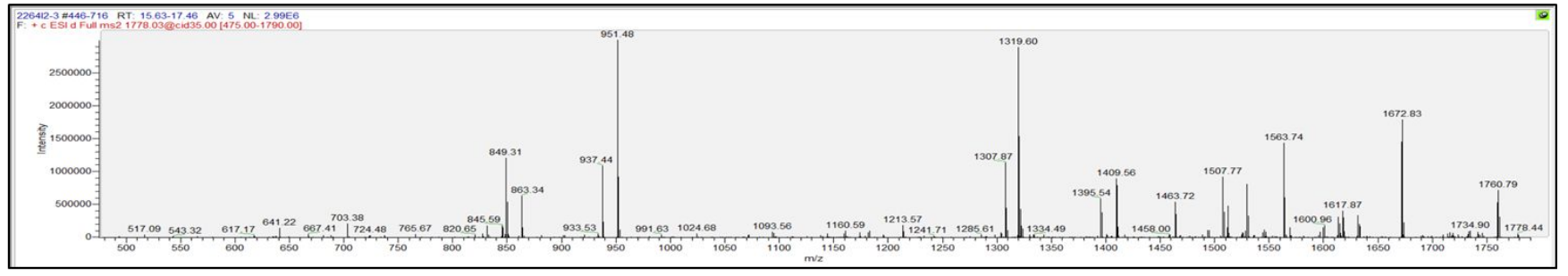

c)

mzspec:GNPSTASK-c4ef17ab0f1a40a1a97bbef42a898549:spectra/specs_ms.mgf:scan:10096

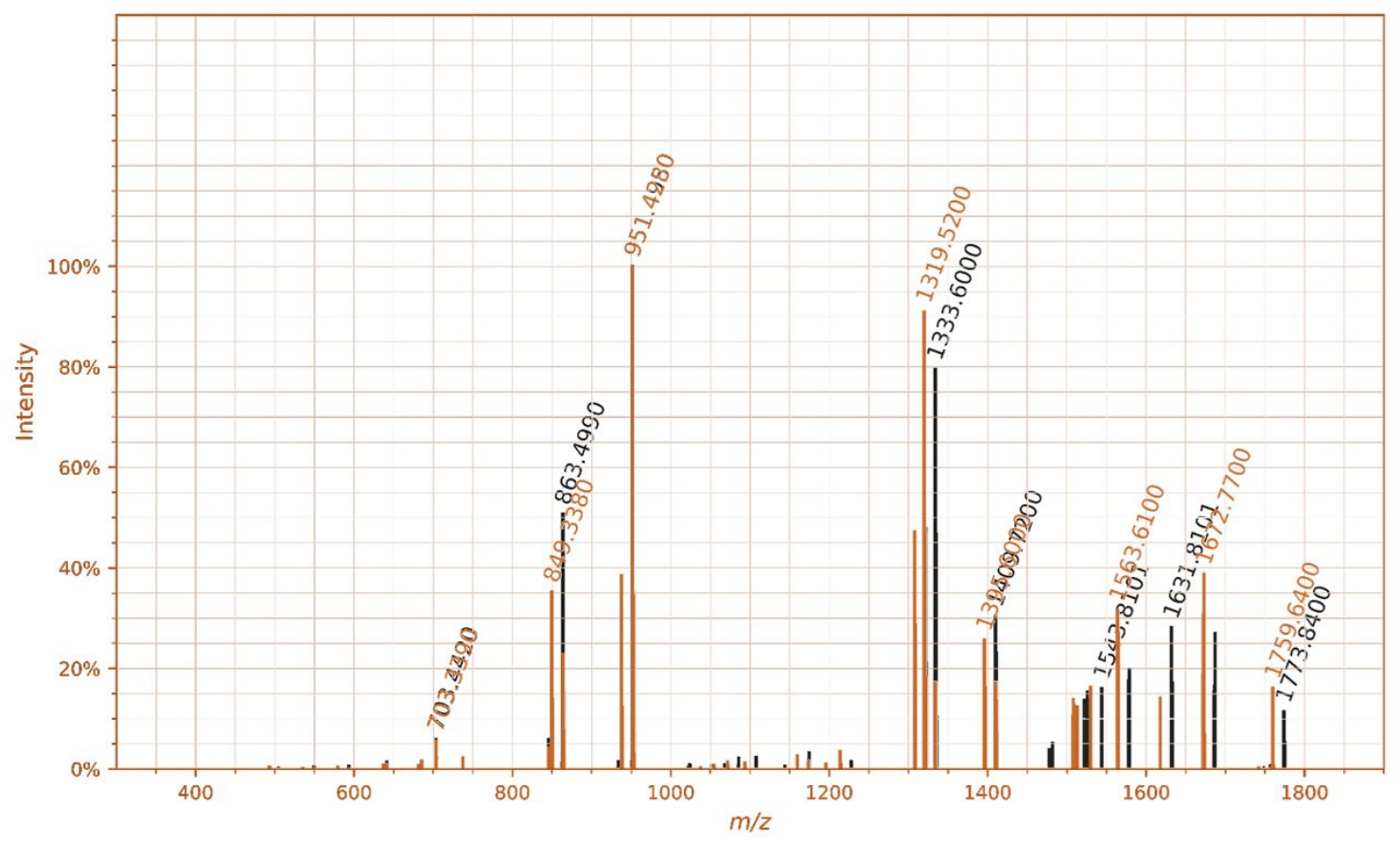

Figure S24. (a) ESI iontrap $\mathrm{MS}^{2}$ spectra $[\mathrm{M}+\mathrm{Na}]^{+}$of samholide E/F/G (black) and (b) putatively new Samholide K (marron, $m / z=1778.8$ ). (c) Overlay plot of a) + b) (same study different $\mathrm{MS}^{2}$ scans). 


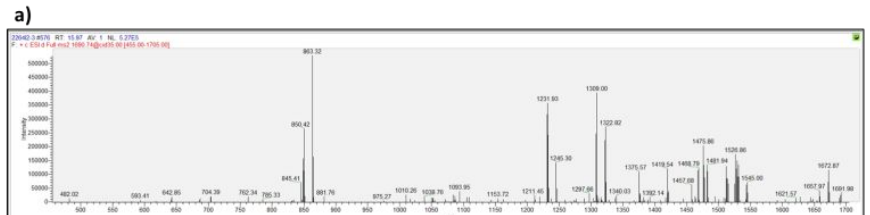

a2)
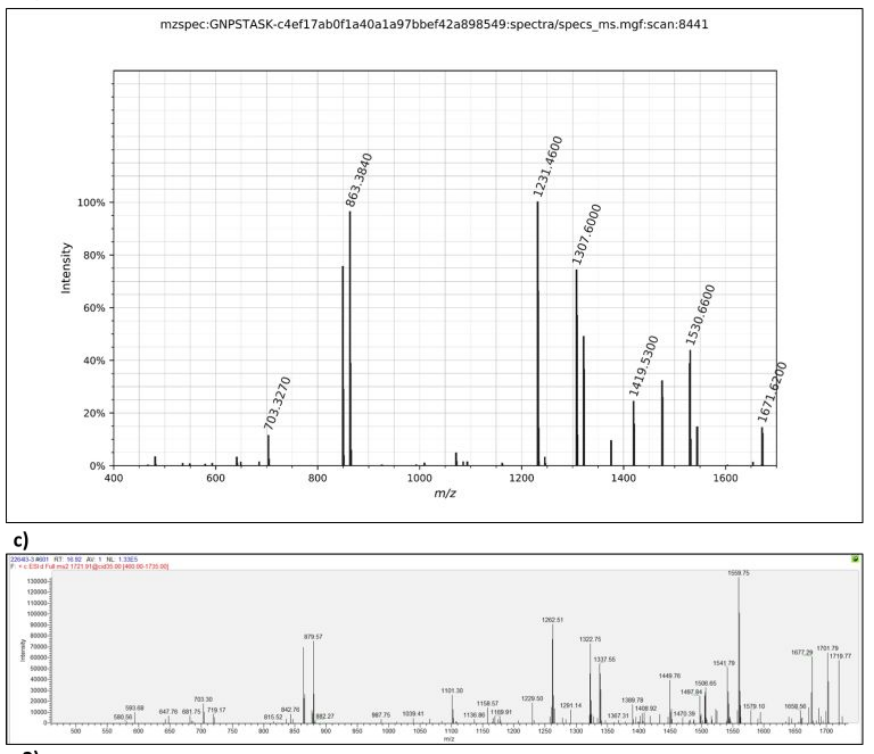

c2)

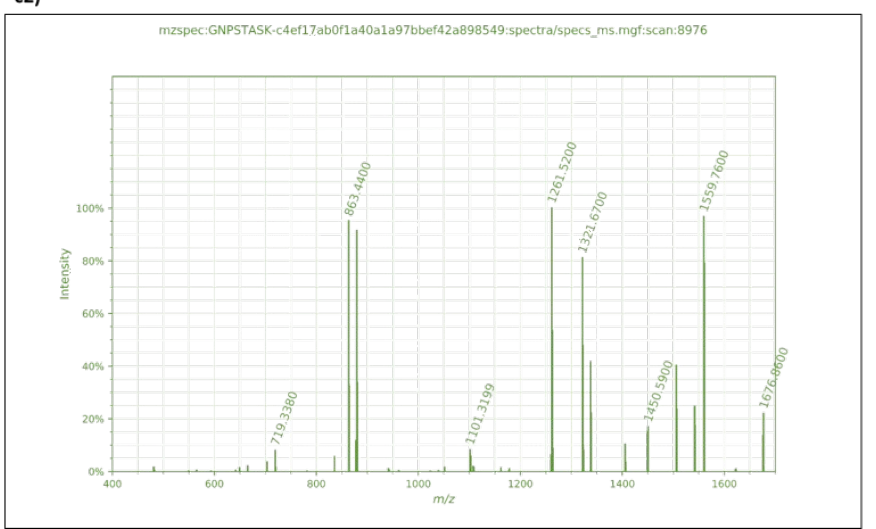

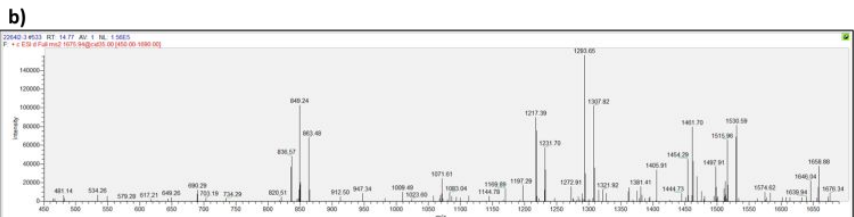

b2)
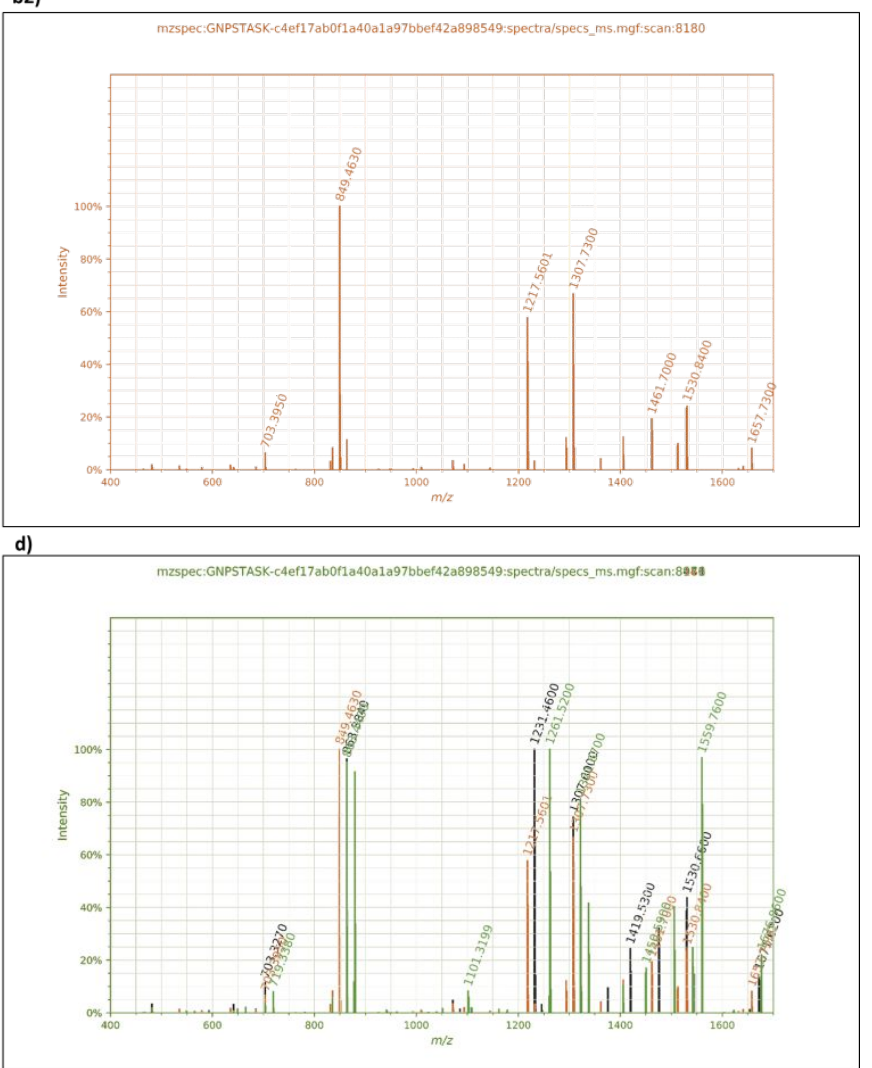

Figure S25. (a,az) ESI iontrap MS² spectra $[\mathrm{M}+\mathrm{Na}]^{+}$of samholide H (black, $m / z=1690.9$ ) and (b,b2) putatively new Samholide L (marron, $m / z=1676.9$ ), and (c) putatively new samholide $M$ (green, $m / z=1721.9$ ) (d) Overlay plot of a2), b2), and c2). 


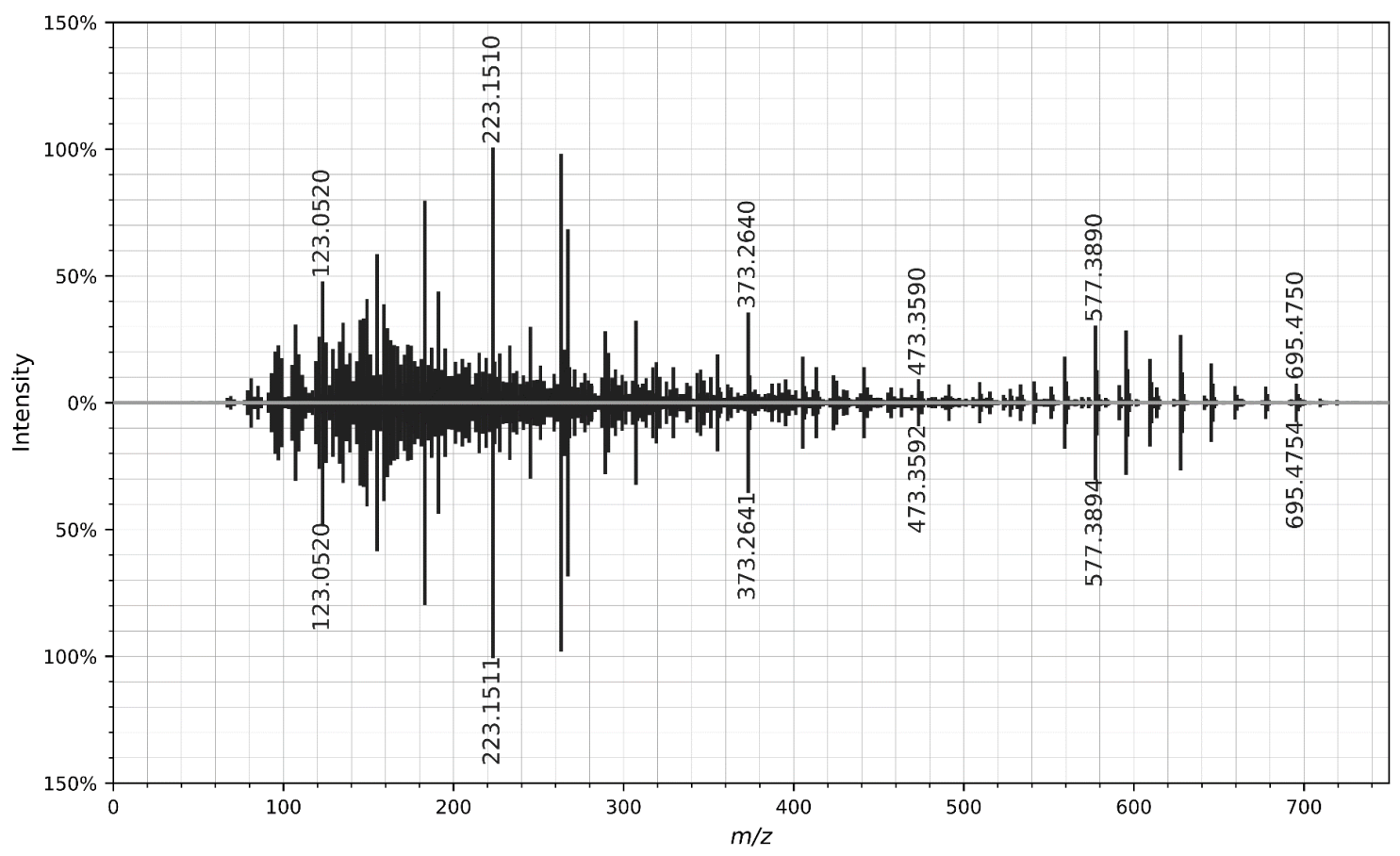

Figure S26. (a) Mirror plot of high-resolution ESI q-ToF MS² spectrum (top) and GNPS library spectrum [M+H]+ of putative swinholide $\mathrm{F}$ (bottom). $\mathrm{m} / \mathrm{z}$ range was set to fingerprint region o-75o Da.

mzspec:GNPSTASK-e41860bffa6f4a7fa3c347a96a698702:spectra/specs_ms.mgf:scan:352=mzspec:GNPSLIBRARY:CCMSLIB00005467780

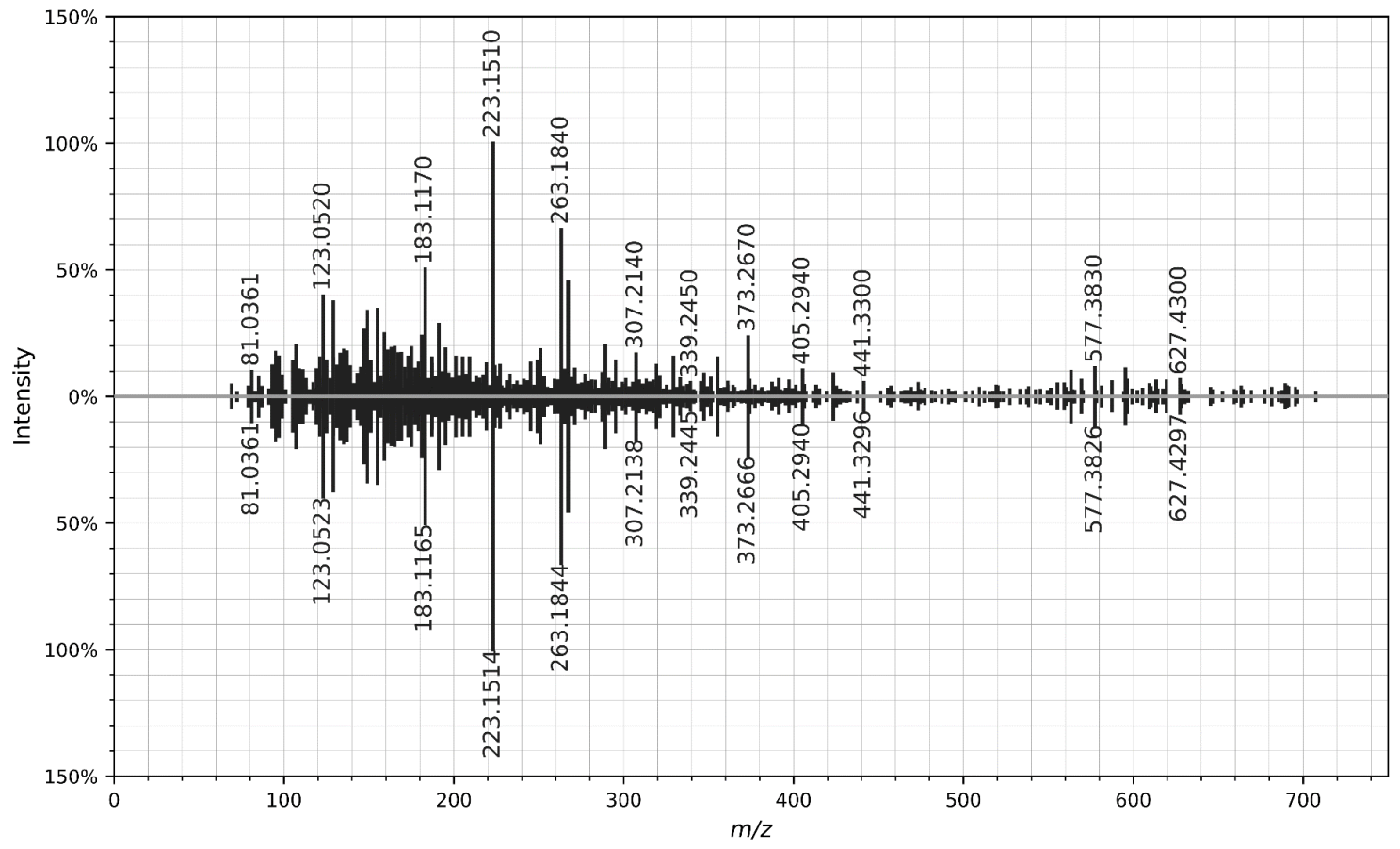

Figure S27. (a) Mirror plot of high-resolution ESI q-ToF MS² spectrum (top) and GNPS library spectrum [M+H] $]^{+}$of putative swinholide $\mathrm{G}$ (bottom). $\mathrm{m} / \mathrm{z}$ range was set to fingerprint region o-75o Da. 


\section{a)}

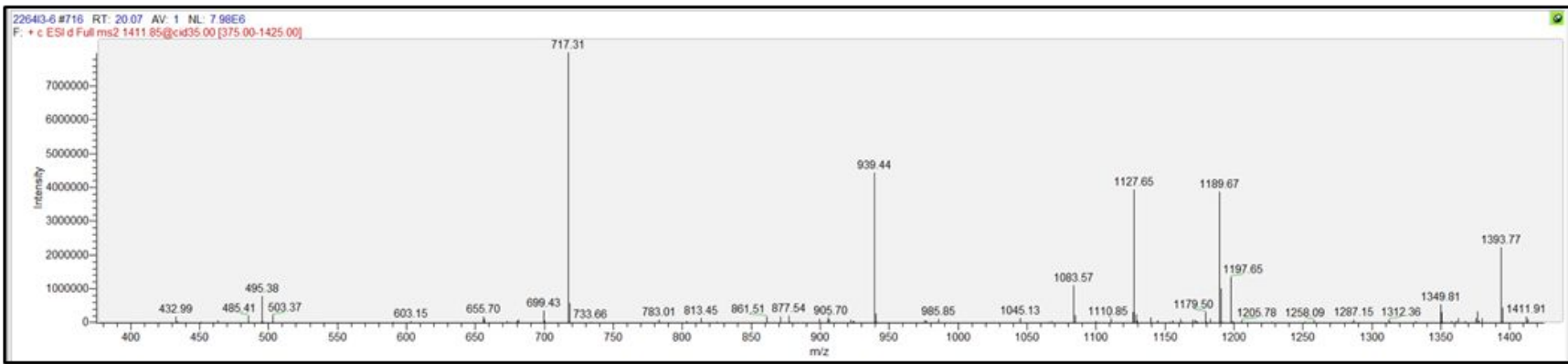

\section{b)}

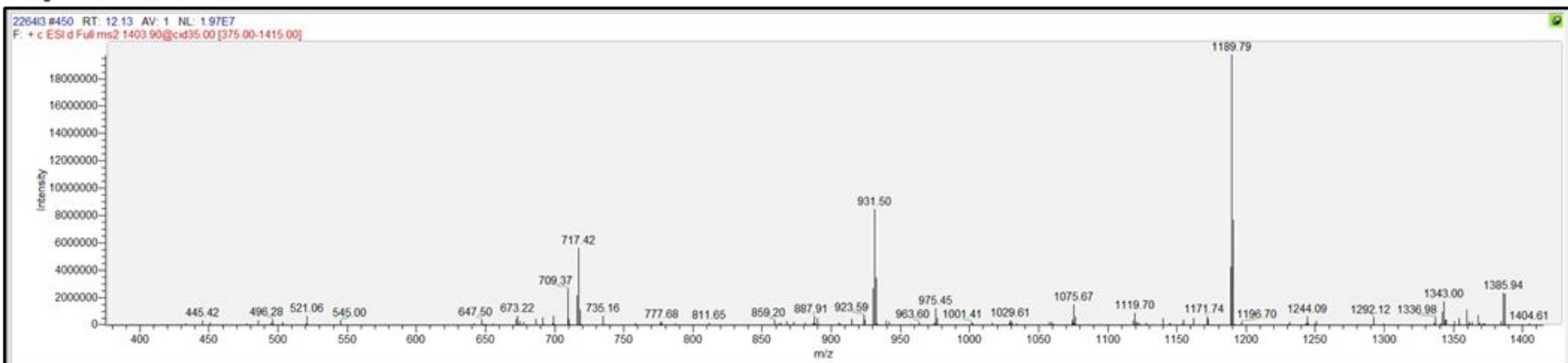

\section{c)}

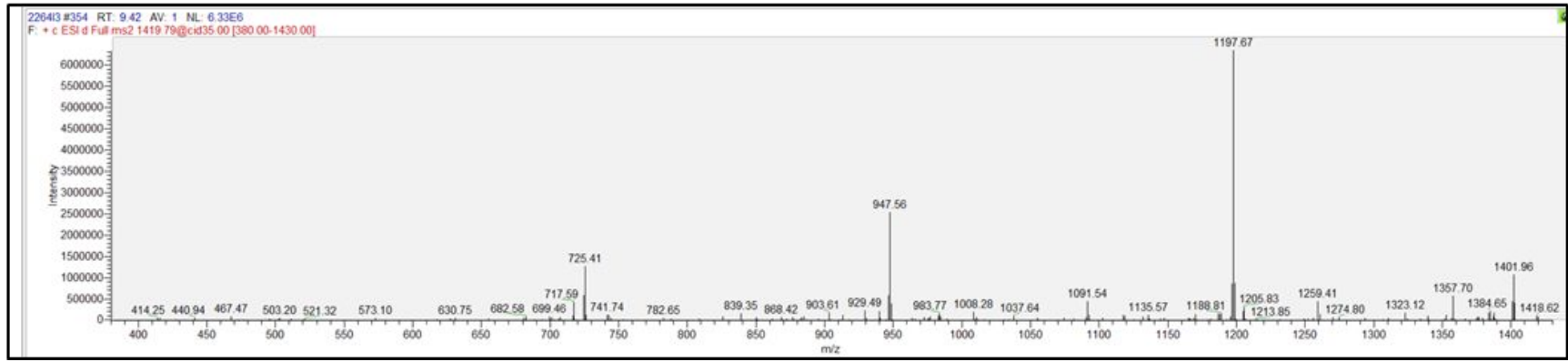

Figure S28. (a) ESI iontrap MS2 spectra $[\mathrm{M}+\mathrm{Na}]^{+}$of swinholide A, putatively new $\mathrm{m} / \mathrm{z}=1403.9$ swinholide $\mathrm{L}$ (b), and putatively new $\mathrm{m} / \mathrm{z}=1419.8$ swinholide $\mathrm{M}$ from this study. 


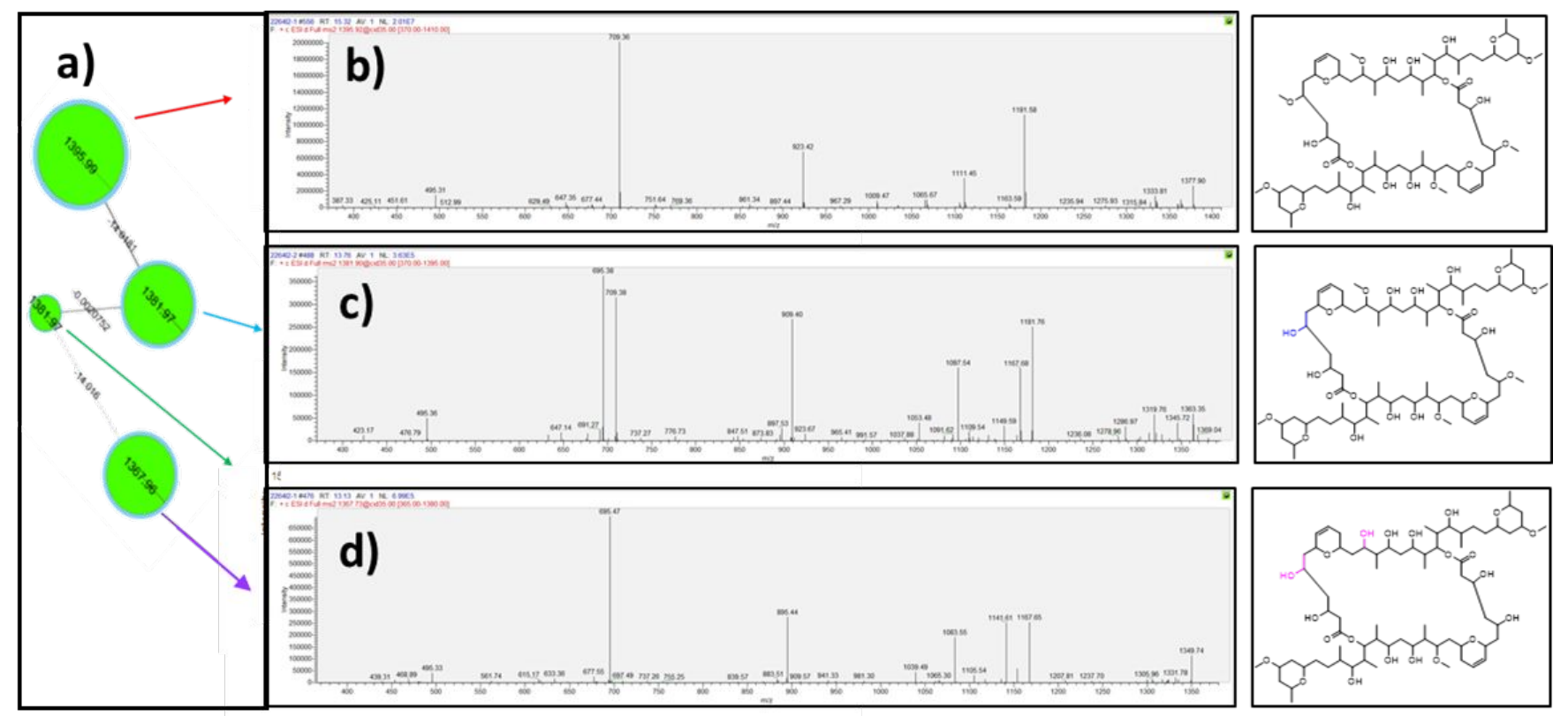

Figure S29. (a) Cluster of symplocolide A and putatively new symplocolide B, and C. (b) ESI iontrap MS2 spectra [M+Na] ${ }^{+}$of symplocolide A ( $m / z=1381.9)$, (c) putatively new $m / z=1381.9$ symplocolide B and (d) putatively new symplocolide $C(\mathrm{~m} / \mathrm{z}=1367.9)$ from this study.

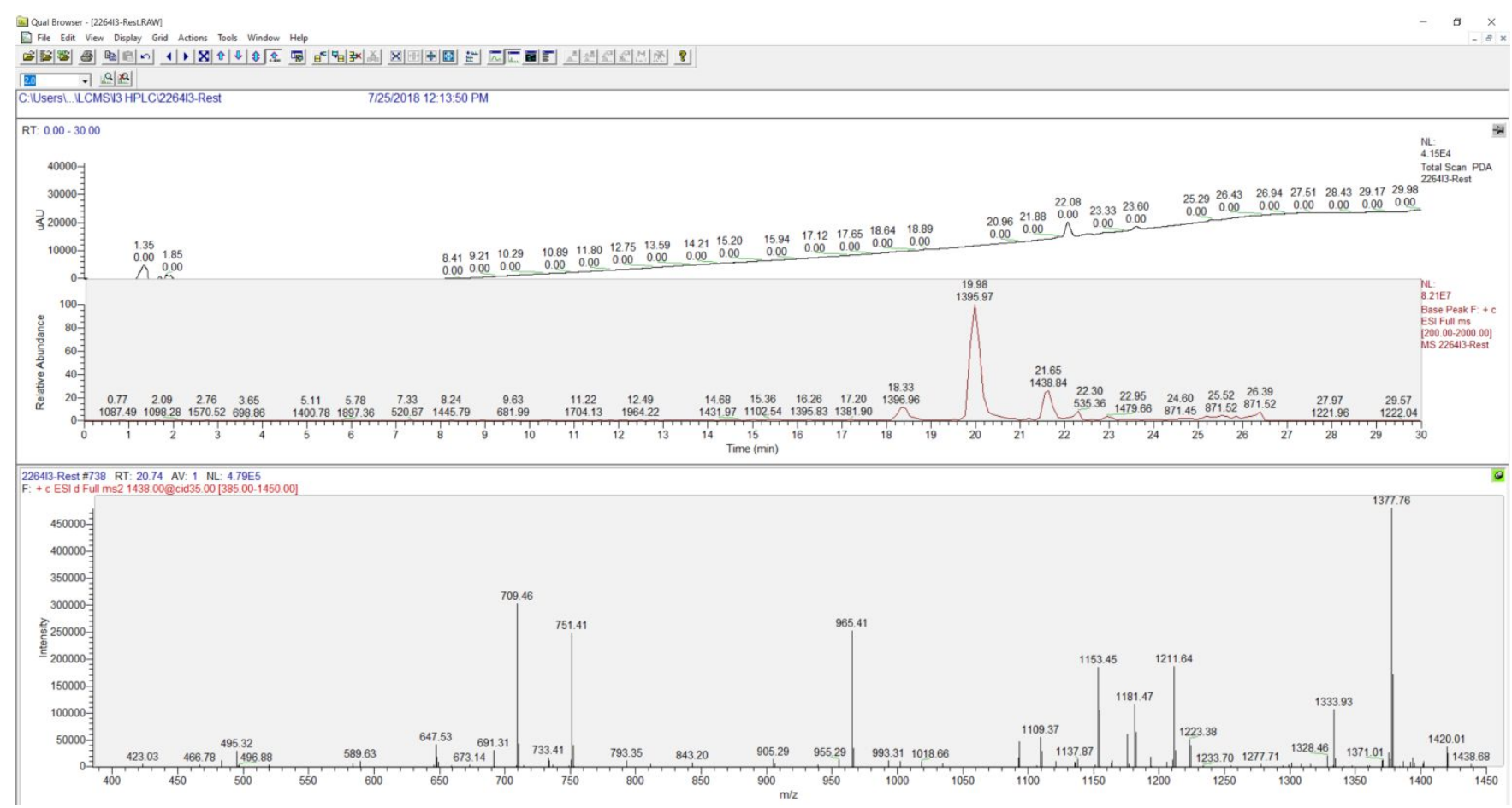

Figure S30. (a) ESI iontrap MS2 spectra $[\mathrm{M}+\mathrm{Na}]^{+}$of putatively new symplocolide $\mathrm{D}(\mathrm{m} / \mathrm{z}=1438.0)$. 


\section{Symplocolide A}

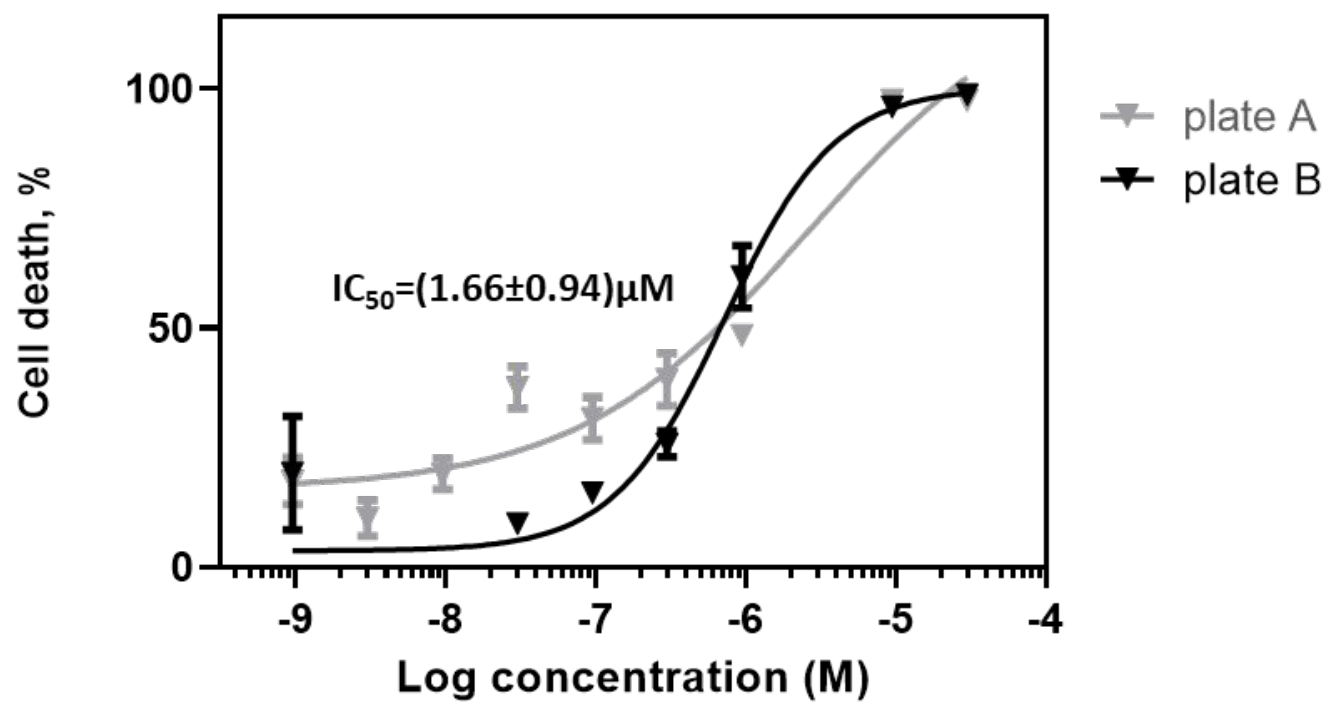

Figure S31. Dose - response graphs gave an $\mathrm{IC}_{50}$ value of $1.66 \pm 0.94 \mu \mathrm{M}$ for symplocolide A and were received using GraphPad Prism version 8.o.o for Windows, GraphPad Software, San Diego, California USA, www.graphpad.com based on nine concentrations tested in duplicates and on two plates.

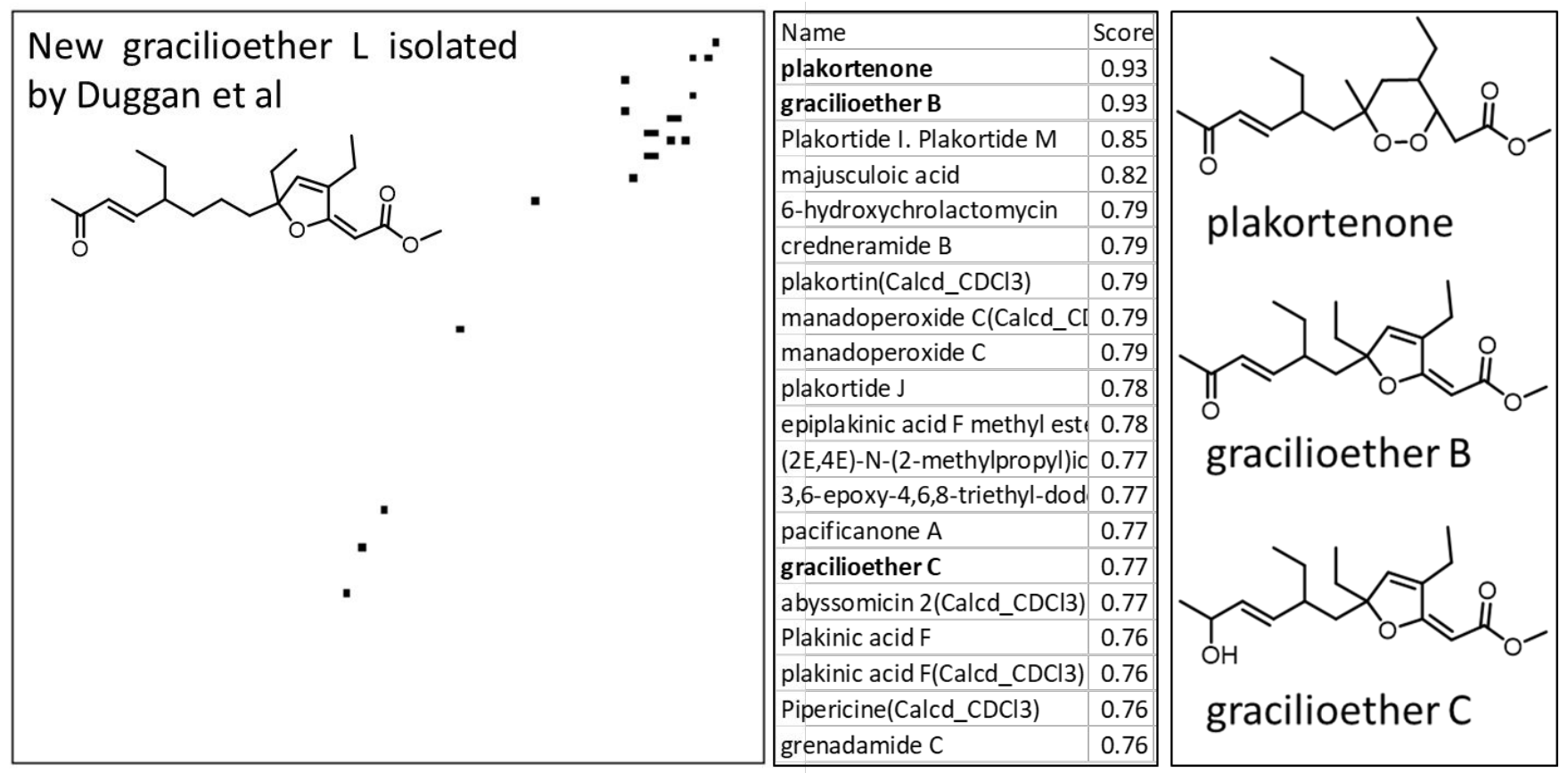

Figure S32. SMART 2.o results for the newly isolated gracilioether L isolated by Duggan et al. ${ }^{20}$ 
a)

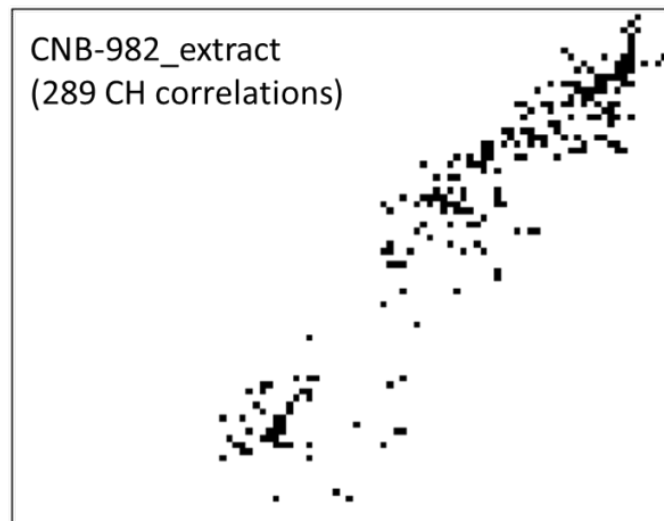

b)

b) HSQC spectrum of Cyclomarin A (MeOD) isolated from CNB-982 by Duggan et al

\begin{tabular}{|l|r|}
\hline Name & Score \\
RP-1776 & 0.83 \\
perthamide C & 0.81 \\
\hline mirabamide H(Calcd_CDCl3 & 0.8 \\
mutremdamide A & 0.79 \\
stellatolide F(Calcd_CDCI3) & 0.79 \\
\hline papuamide E(Calcd_CDCI3) & 0.79 \\
mirabamide F(Calcd_CDCI3 & 0.79 \\
papuamide F(Calcd_CDCI3) & 0.78 \\
mirabamide E(Calcd_CDCl3 & 0.78 \\
stellatolide G(Calcd_CDCI3) & 0.77 \\
mirabamide B(Calcd_CDCl3 & 0.77 \\
callipeltin H(Calcd_CDCl3) & 0.77 \\
RA-XXI & 0.77 \\
\hline mirabamide E & 0.76 \\
\hline mirabamide A & 0.74 \\
\hline [D-Leu]-microcystin-LR & 0.74 \\
\hline lyngbyastatin 6(Calcd_CDCl & 0.74 \\
ilamycin C2(Calcd_CDCI3) & 0.74 \\
\hline ilamycin C1(Calcd_CDCI3) & 0.74 \\
\hline largamide H & 0.74 \\
\hline
\end{tabular}

largamide $\mathrm{H}$

\begin{tabular}{|l|r|}
\hline Name & Score \\
\hline ilamycin C1(Calcd_CDCl3) & 0.83 \\
\hline ilamycin C2(Calcd_CDCl3) & 0.82 \\
\hline Phakellistatin 13(Calcd_CD & 0.8 \\
\hline kapakahine E(Calcd_CDCl3) & 0.8 \\
\hline KB425796-A & 0.79 \\
\hline phakellistatin 15(Calcd_CDI & 0.79 \\
\hline brachystemin G & 0.78 \\
\hline CHEMBL413654(Calcd_CDC & 0.78 \\
\hline hymenamide H(Calcd_CDCl & 0.78 \\
\hline CHEMBL3787164(Calcd_CC & 0.78 \\
\hline microcystin WA & 0.78 \\
\hline corticiamide A & 0.78 \\
\hline A21978C1-3(D-Asn11) & 0.78 \\
\hline AxinellinC & 0.77 \\
\hline (+)-Jasplakinolide Z2(Calcd_ & 0.77 \\
\hline cyclomontanin A(Calcd_CD & 0.77 \\
\hline perthamide C & 0.77 \\
\hline pahayokolide B & 0.77 \\
\hline mutremdamide A & 0.77 \\
\hline perthamide G & 0.77 \\
\hline
\end{tabular}

perthamide $G$

\begin{tabular}{|l|r|}
\hline Name & Score \\
\hline (+)-Jasplakinolide Z2(Calcd_CD & 0.73 \\
\hline (+)-jasplakinolide Z2 & 0.71 \\
\hline Apicidin D2 & 0.7 \\
\hline cyclomarin D(Calcd_CDCI3) & 0.7 \\
\hline jasplakinolide Z3(Calcd_CDCI3) & 0.69 \\
\hline MBJ-0039 & 0.69 \\
\hline (+)-jasplakinolide Z1 & 0.68 \\
\hline cyclomarin C(Calcd_CDC13) & 0.68 \\
\hline tauramamide ethyl ester(Calcd & 0.68 \\
\hline (+)-jasplakinolide Z3 & 0.67 \\
\hline ilamycin C2(Calcd_CDC13) & 0.67 \\
\hline Jasplakinolide D(Calcd_CDCl3) & 0.67 \\
\hline symplocin A & 0.67 \\
\hline Phakellistatin 13(Calcd_CDCI3) & 0.67 \\
\hline (+)-Jasplakinolide Z1(Calcd_CD & 0.67 \\
\hline jaspamide M(Calcd_CDC13) & 0.67 \\
\hline MBJ-0038 & 0.67 \\
\hline Apicidin D1 & 0.67 \\
\hline ilamycin C1(Calcd_CDC13) & 0.66 \\
\hline kahalalide V & 0.66 \\
\hline
\end{tabular}
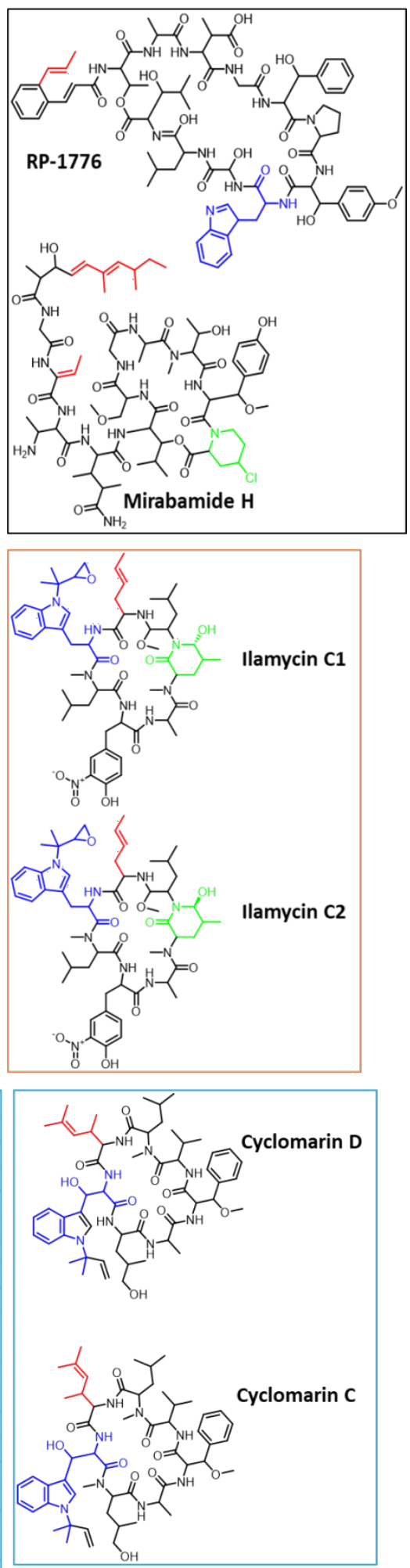

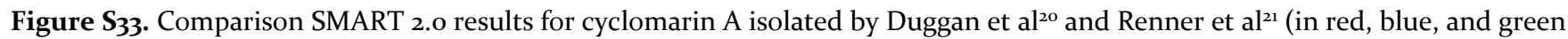
colors highlight rare structural moieties detected by SMART for Cyclomarin A and related compound, Cyclomarin A was isolated guided by atomic sort method focusing on one unusual highfield chemical shift of a shielded diasterotopic methylene proton (interestingly the chemical shift reported by Duggan et al differed by $>1.5 \mathrm{ppm}\left(\mathrm{H}-5 \mathrm{lb}=-0.9 \mathrm{ppm}, \mathrm{CD}_{3} \mathrm{OD}\right)$ from the originally reported shift $\left(\mathrm{H}-51 \mathrm{~b}=0.72 \mathrm{ppm}\right.$, Renner et al, $\left.\mathrm{CDCl}_{3}\right)$, presumably due to different adopted conformations for each solvent). a) HSQC spectrum and Top2o SMART results of the cyclomarin A-containing crude extract. b) HSQC spectrum and Top2o SMART results of cyclomarin A isolated by Duggan et al ${ }^{20}$ measured in MeOD. c) HSQC spectrum and Top2o SMART results of cyclomarin A isolated by Renner et al ${ }^{21}$ measured in $\mathrm{CDCl}_{3}$. 


\section{REFERENCES}

(1) Tao, Y.; Li, P.; Zhang, D.; Glukhov, E.; Gerwick, L.; Zhang, C.; Murray, T. F.; Gerwick, W. H. Samholides, Swinholide-Related Metabolites from a Marine Cyanobacterium Cf. Phormidium Sp. The Journal of Organic Chemistry 2018, 83 (6), 3034-3046. https://doi.org/10.1021/acs.joc.8booo28.

(2) Kitagawa, I.; Kobayashi, M.; Katori, T.; Yamashita, M.; Tanaka, J.; Doi, M.; Ishida, T. Absolute Stereostructure of Swinholide A, a Potent Cytotoxic Macrolide from the Okinawan Marine Sponge Theonella Swinhoei. J. Am. Chem. Soc. 1990, 112 (9), 3710-3712. https://doi.org/10.1021/ja00165aog4.

(3) Ishibashi, M.; Moore, R. E.; Patterson, G. M. L.; Xu, C.; Clardy, J. Scytophycins, Cytotoxic and Antimycotic Agents from the Cyanophyte Scytonema Pseudohofmanni. J. Org. Chem. 1986, 51 (26), 5300-5306. https://doi.org/10.1021/j000376a047.

(4) Nicolaou, K. C.; Ajito, K.; Patron, A. P.; Khatuya, H.; Richter, P. K.; Bertinato, P. Total Synthesis of Swinholide A. J. Am. Chem. Soc. 1996, 118 (12), 3059-306o. https://doi.org/10.1021/ja954211t.

(5) Ueoka, R.; Uria, A. R.; Reiter, S.; Mori, T.; Karbaum, P.; Peters, E. E.; Helfrich, E. J. N.; Morinaka, B. I.; Gugger, M.; Takeyama, $\mathrm{H}_{\text {; }}$ et al. Metabolic and Evolutionary Origin of Actin-Binding Polyketides from Diverse Organisms. Nature Chemical Biology 2015, 11 (9), 705-712. https://doi.org/10.1038/nchembio.1870.

(6) Humisto, A.; Jokela, J.; Liu, L.; Wahlsten, M.; Wang, H.; Permi, P.; Machado, J. P.; Antunes, A.; Fewer, D. P.; Sivonen, K. The Swinholide Biosynthesis Gene Cluster from a Terrestrial Cyanobacterium, Nostoc Sp. Strain UHCC 0450. Applied and Environmental Microbiology 2017, 84 (3). https://doi.org/10.1128/AEM.02321-17.

(7) Andrianasolo, E. H.; Gross, H.; Goeger, D.; Musafija-Girt, M.; McPhail, K.; Leal, R. M.; Mooberry, S. L.; Gerwick, W. H. Isolation of Swinholide A and Related Glycosylated Derivatives from Two Field Collections of Marine Cyanobacteria. Organic Letters 2005, 7 (7), 1375-1378. https://doi.org/10.1021/olo50188x.

(8) Kessner, D.; Chambers, M.; Burke, R.; Agus, D.; Mallick, P. ProteoWizard: Open Source Software for Rapid Proteomics Tools Development. Bioinformatics 2008, $24 \quad$ (21), 2534-2536. https://doi.org/10.1093/bioinformatics/btn323.

(9) Wang, M.; Carver, J. J.; Phelan, V. V.; Sanchez, L. M.; Garg, N.; Peng, Y.; Nguyen, D. D.; Watrous, J.; Kapono, C. A.; Luzzatto-Knaan, T.; et al. Sharing and Community Curation of Mass Spectrometry Data with Global Natural Products Social Molecular Networking. Nature Biotechnology 2016, 34 (8), 828-837. https://doi.org/10.1038/nbt.3597.

(10) Nothias, L. F.; Petras, D.; Schmid, R.; Dührkop, K.; Rainer, J.; Sarvepalli, A.; Protsyuk, I.; Ernst, M.; Tsugawa, H.; Fleischaver, M.; et al. Feature-Based Molecular Networking in the GNPS Analysis Environment. bioRxiv 2019, 812404. https://doi.org/10.1101/812404.

(11) Röst, H. L.; Sachsenberg, T.; Aiche, S.; Bielow, C.; Weisser, H.; Aicheler, F.; Andreotti, S.; Ehrlich, H.-C.; Gutenbrunner, P.; Kenar, E.; et al. OpenMS: A Flexible Open-Source Software Platform for Mass Spectrometry Data Analysis. Nature Methods 2016, 13 (9), 741-748. https://doi.org/10.1038/nmeth.3959.

(12) Shannon, P.; Markiel, A.; Ozier, O.; Baliga, N. S.; Wang, J. T.; Ramage, D.; Amin, N.; Schwikowski, B.; Ideker, T. Cytoscape: A Software Environment for Integrated Models of Biomolecular Interaction Networks. Genome Res. 2003, 13 (11), 2498-2504. https://doi.org/10.1101/gr.1239303.

(13) Scudiero, D. A.; Shoemaker, R. H.; Paull, K. D.; Monks, A.; Tierney, S.; Nofziger, T. H.; Currens, M. J.; Seniff, D.; Boyd, M. R. Evaluation of a Soluble Tetrazolium/Formazan Assay for Cell Growth and Drug Sensitivity in Culture Using Human and Other Tumor Cell Lines. Cancer Res 1988, 48 (17), 4827-4833.

(14) [1602.07360] SqueezeNet: AlexNet-level accuracy with 50x fewer parameters and <0.5MB model size https://arxiv.org/abs/1602.07360 (accessed Dec 17, 2019).

(15) Deng, J.; Dong, W.; Socher, R.; Li, L.-J.; Kai Li; Li Fei-Fei. ImageNet: A Large-Scale Hierarchical Image Database. In 2009 IEEE Conference on Computer Vision and Pattern Recognition; 2009; pp 248-255. https://doi.org/10.1109/CVPR.2009.5206848.

(16) Dehghani, M.; Gouws, S.; Vinyals, O.; Uszkoreit, J.; Kaiser, t. UNIVERSAL TRANSFORMERS. 2019, 23.

(17) Rogers, D.; Hahn, M. Extended-Connectivity Fingerprints. J. Chem. Inf. Model. 2010, 50 (5), $742-754$. https://doi.org/10.1021/ci100050t.

(18) Pereira, F.; Aires-de-Sousa, J. Computational Methodologies in the Exploration of Marine Natural Product Leads. Marine Drugs 2018, 16 (7), 236. https://doi.org/10.3390/md16070236.

(19) Youssef, D. T. A.; Mooberry, S. L. Hurghadolide A and Swinholide I, Potent Actin-Microfilament Disrupters from the Red Sea Sponge Theonella s Winhoei. J. Nat. Prod. 2006, 69 (1), 154-157. https://doi.org/10.1021/np050404a. 
(20) Duggan, B. M.; Cullum, R.; Fenical, W.; Amador, L. A.; Rodríguez, A. D.; La Clair, J. J. Searching for Small Molecules with an Atomic Sort. Angew. Chem. Int. Ed. 2020, 59, 1144-1148.

(21) Renner, M. K.; Shen, Y.-C.; Cheng, X.-C.; Jensen, P. R.; Frankmoelle, W.; Kauffman, C. A.; Fenical, W.; Lobkovsky, E.; Clardy, J. Cyclomarins A-C, New Antiinflammatory Cyclic Peptides Produced by a Marine Bacterium (Streptomyces Sp.). J. Am. Chem. Soc. 1999, 121 (49), 11273-11276. https://doi.org/10.1021/jag924820. 